\title{
A NOVEL APPROACH FOR TREATING RESISTANT HYPERTENSION USING A CONTROLLED-PUMP ACCUMULATOR RELIEF DEVICE
}

\section{A Dissertation}

presented to

The Faculty of the Graduate School

At the University of Missouri - Columbia

In Partial Fulfillment

of the Requirements for the Degree

Doctor of Philosophy

by

ADEL M. ALTURKI

Dr. Noah Manring, Dissertation Supervisor

May 2018 
(C) Copyright by Adel M. Alturki 2018

\author{
All Rights Reserved
}


The undersigned, appointed by the dean of the Graduate School, have examined the

Dissertation entitled

\section{A NOVEL APPROACH FOR TREATING RESISTANT HYPERTENSION \\ USING A CONTROLLED-PUMP ACCUMULATOR RELIEF DEVICE}

Presented by

Adel M. Alturki

A candidate for the degree of Doctor of Philosophy in

Electrical and Computer Engineering

And hereby certify that, in their opinion, it is worthy of acceptance.

Professor. Noah D. Manring

Professor. Robert O’Connell

Professor. Roger Fales

Professor. Craig A. Emter 


\section{ACKNOWLEDGEMENTS}

First, I would like to express my sincere gratitude to my advisor, Dr. Noah Manring, for his endless support and guidance and for affording me the opportunity to perform this body of work. Dr. Manring is not just an advisor, but he is also a great person and real leader. I would also like to extend my sincere gratitude to the members of my dissertation committee: Dr. Roger Fales, Dr. Craig Emter, and Dr. Robert O’Connell for serving on my committee and for their valuable comments.

I would also like to thank my sponsor, the Royal Commission for Jubail and Yanbu, for granting me a fully-funded scholarship to pursue my graduate studies in the United States. Their support has greatly helped me focus on my research and study. I am also grateful to my friends who I knew during my study at Mizzou for their real friendship.

I owe a great debt of gratitude to the University of Missouri library system, especially the ILL office, for the great wealth of knowledge and other tools to which I had access throughout my studies.

Special thanks go to my parent and siblings for their love and support. They are one of the biggest blessings in my life.

Finally, I am particularly grateful to my wonderful wife, Wafaa, and children - Tala, Abdulaziz, and Deema - who were my companions throughout this $\mathrm{PhD}$ journey. Without their constant love, patience, and continued support and encouragement, I could not have accomplished this achievement. 


\section{TABLE OF CONTENTS}

ACKNOWLEDGEMENTS .................................................................................. ii

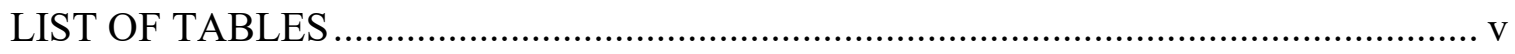

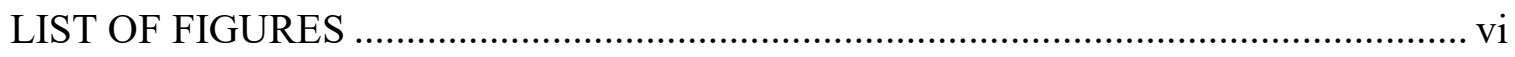

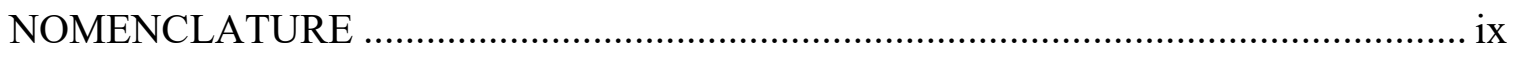

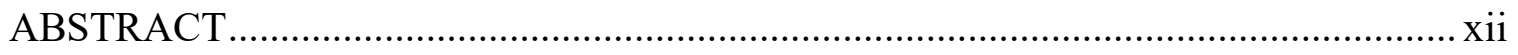

CHAPTER 1 Introduction................................................................................... 1

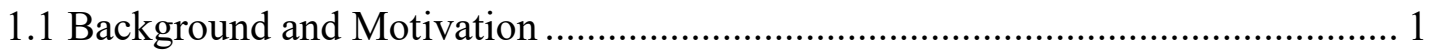

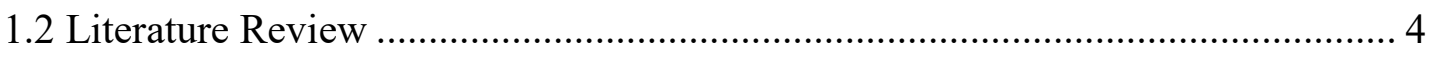

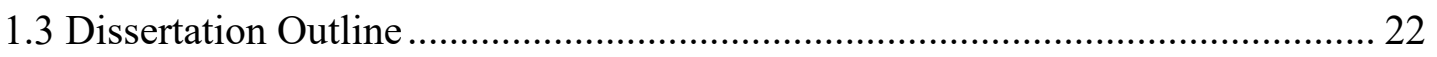

CHAPTER 2 Description of the Cardiovascular System ............................................. 23

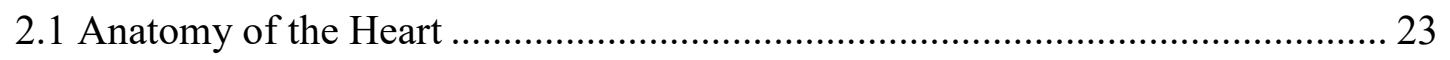

2.2 Cardiac Cycle Phases.................................................................................. 25

2.3 Pressure-Volume (PV) Loop and System Hemodynamics................................ 29

CHAPTER 3 Modeling the Cardiovascular System ...................................................... 35

3.1 Analogies between the Circulatory System and Electric Circuits ...................... 35

3.1.1 Modeling a Vessel .......................................................................... 36

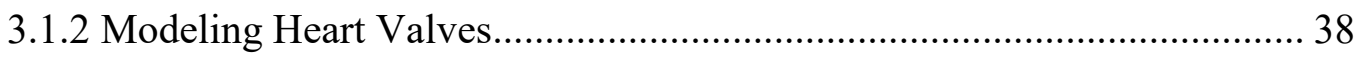

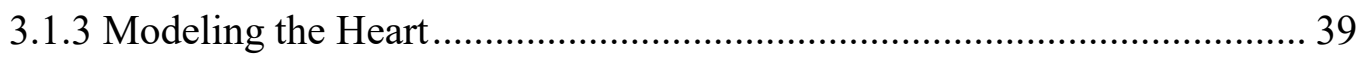

3.1.4 Modeling the Arterial Network ........................................................ 42

3.2 Lumped-Parameter Model of the Cardiovascular System ................................. 43

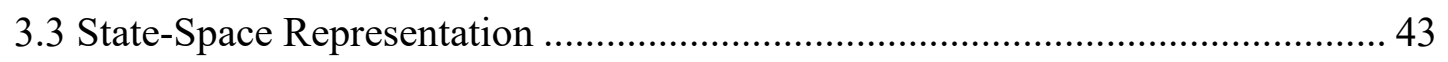

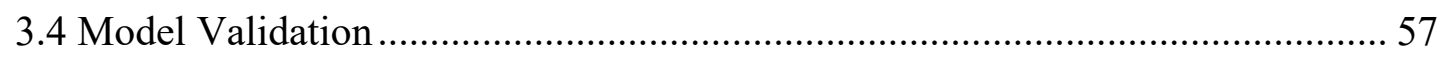

3.4.1 Model Validation by Reproducing Expected Hemodynamics ................. 57

3.4.2 Model Validation by Varying Preload and Afterload Conditions............. 59

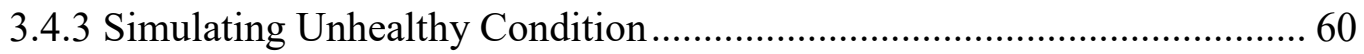

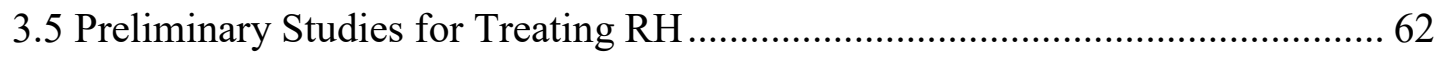

3.5.1 Using CCM to Decrease the Cardiac Contractility ................................6 62

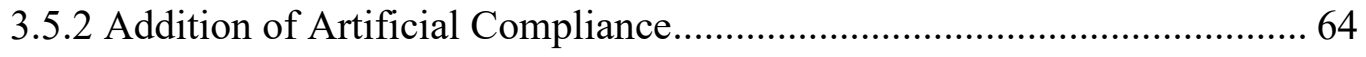

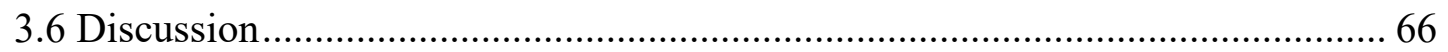




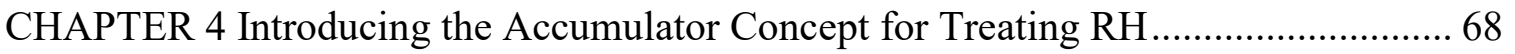

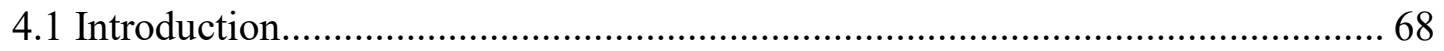

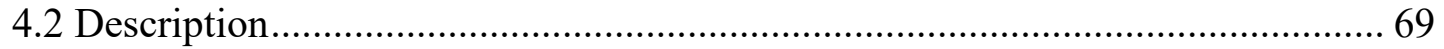

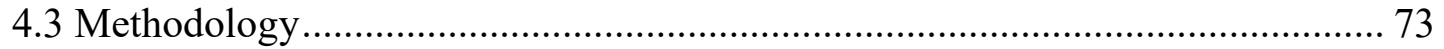

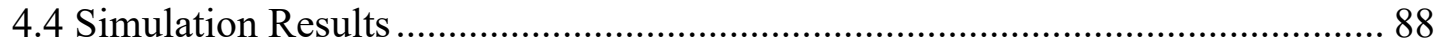

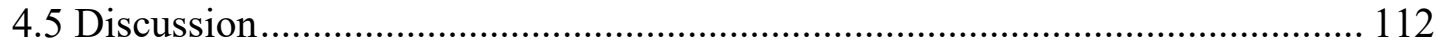

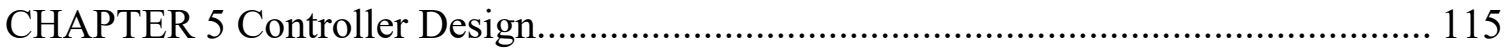

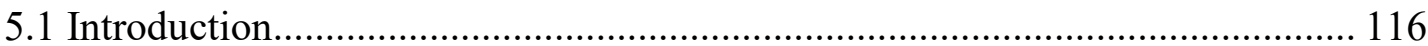

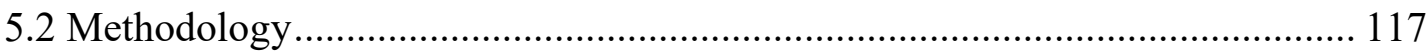

5.3 Simulation Results and Discussion............................................................. 124

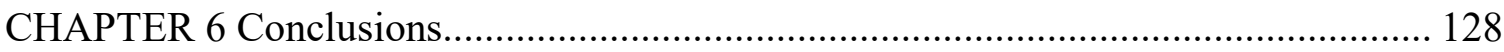

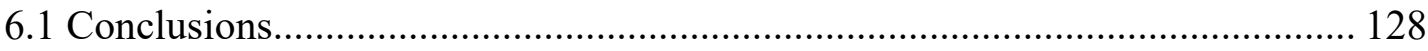

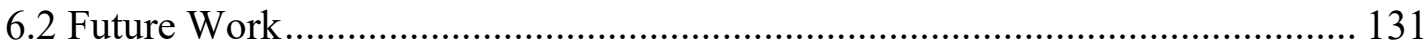

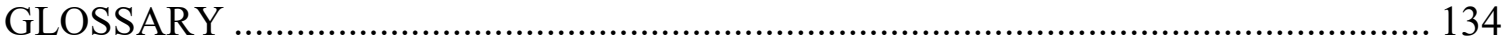

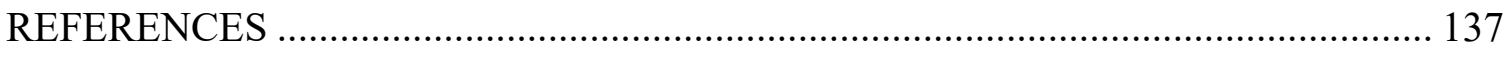

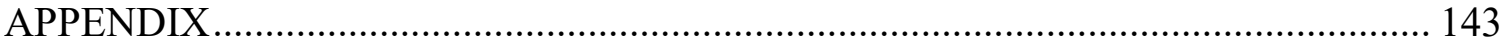

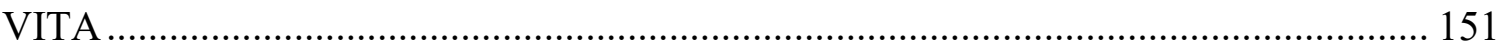




\section{LIST OF TABLES}

Tables

Page

\section{Chapter 3}

Table 3.1 Cardiac cycle phases: valve states and time duration .................. 40

Table 3.2 State variables used in the cardiovascular system model .................... 44

Table 3.3 Parametric values of the hemodynamical elements used in the

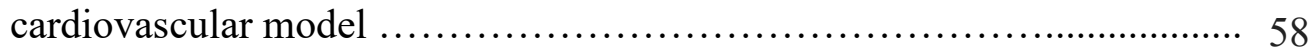

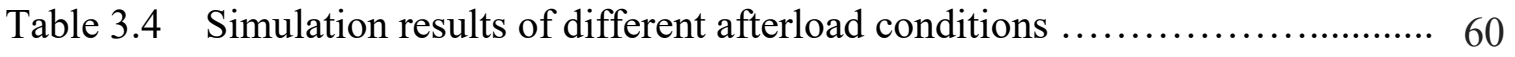

Table 3.5 Effect of applying negative CCM signals .......................... 63

Table 3.6 Effect of increasing heart rate while applying negative CCM

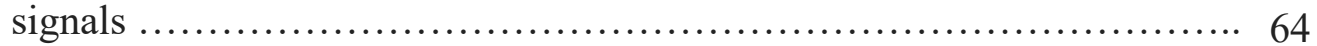

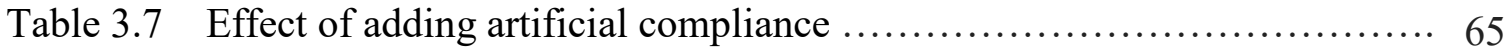

\section{Chapter 4}

Table 4.1 Accumulator status during each phase of the cardiac cycle ................. 72

Table 4.2 State variables of the cardiovascular system with accumulator ................. 73

Table 4.3 Hemodynamics for cases of different values for accumulator resistance

Table 4.4 Hemodynamics for cases of different values for accumulator resistance

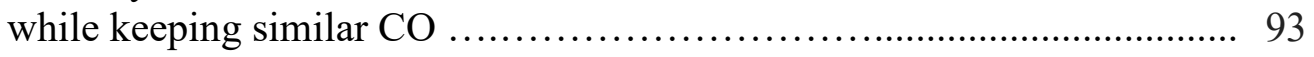

Table 4.5 Influence of changing $\mathrm{R}_{\mathrm{i}}$ value on cardiac cycle phases duration .............. 103

\section{Chapter 5}

Table 5.1 Controller performance in tracking desired reference signals 126 


\section{LIST OF FIGURES}

Figures $\quad$ Page

Chapter 1

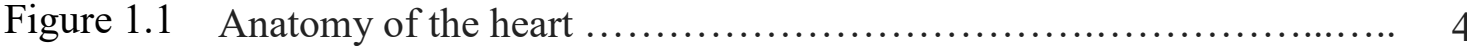

Figure 1.2 Components of a typical LVAD ................................. 8

Figure 1.3 Different pump types of continuous-flow LVADs ................... 9

Figure 1.4 Typical protocol for CCM signals application .................... 11

Figure 1.5 Effects of positive and negative CCM stimuli on contractility .............. 13

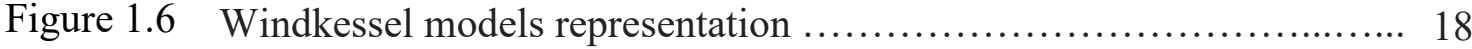

\section{Chapter 2}

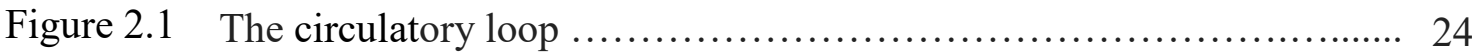

Figure 2.2 Blood movement in arteries during systole and diastole ............... 25

Figure 2.3 Dynamic behaviors during ventricular contraction and relaxation ...... 26

Figure 2.4 Cardiac cycle phases .......................................... 28

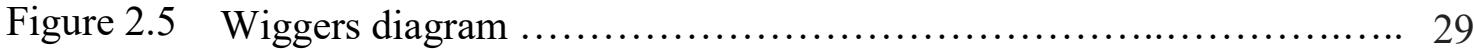

Figure 2.6 Pressure-volume (PV) loop ................................ 30

Figure 2.7 Determining stroke volume from the PV loop ..................... 31

Figure 2.8 Reading systolic/diastolic blood pressure from the PV loop ............ 32

Figure 2.9 Finding ESPVR and EDPVR from the PV loop .................. 33

Figure 2.10 Computing elastance function E(t) from the PV loop ................ 34

\section{Chapter 3}

Figure 3.1 Windkessel representation of the circulatory system $\ldots \ldots \ldots \ldots \ldots \ldots \ldots \ldots$

Figure 3.2 Electrical analogue of vascular elements ......................... 37

Figure 3.3 Modeling heart valves ....................................... 39

Figure 3.4 Time-varying elastance function that models the LV . .............. 42

Figure 3.5 4-element Windkessel model representing the arterial network ........ 42

Figure 3.6 Lumped parameter electric circuit model of the cardiovascular

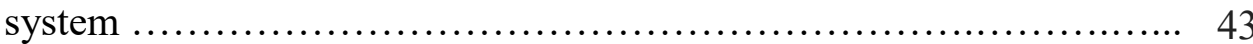

Figure 3.7 Equivalent circuit of the isovolumic contraction and relaxation phases ..................................................... 45

Figure 3.8 Simplified circuit for the isovolumic phases $\ldots \ldots \ldots \ldots \ldots \ldots \ldots \ldots \ldots \ldots \ldots$ 
Figure 3.9 Equivalent circuit of the ejection phase .............................. 49

Figure 3.10 Simplified circuit of the ejection phase .............................. 49

Figure 3.11 Equivalent circuit representing the filling phase ..................... 53

Figure 3.12 Simplified circuit of the filling phase ............................... 54

Figure 3.13 Hemodynamics waveforms of healthy condition ...................... 58

Figure 3.14 PV-loops for different afterload conditions ........................... 60

Figure 3.15 Hemodynamics waveforms of unhealthy condition .................... 61

Figure 3.16 Cardiovascular model with the additional artificial compliance ......... 65

\section{Chapter 4}

Figure 4.1 A schematic describing the accumulator device concept ................ 69

Figure 4.2 Analogy between hydraulic accumulator and electric capacitor......... 70

Figure 4.3 Implementation of the accumulator concept into the cardiovascular system lumped model ............................... 70

Figure 4.4 Equivalent circuit of the cardiovascular system with accumulator device during the isovolumic phases .... 74

Figure 4.5 Simplified circuit model for the cardiovascular system with accumulator device during isovolumic phases

Figure 4.6 Equivalent circuit of the cardiovascular system with accumulator device during ejection phase 76

Figure 4.7 Simplified circuit model for the cardiovascular system with accumulator device during ejection phase

Figure 4.8 Equivalent circuit of the cardiovascular system with accumulator device during filling phase

Figure 4.9 Simplified circuit model for the cardiovascular system with accumulator device during filling phase

Figure 4.10 Simplified circuit model for the cardiovascular system with accumulator device during filling phase with current source

Figure 4.11 Hemodynamics waveforms of baseline condition ..................... 90

Figure 4.12 Influence of accumulator implementation on hemodynamics ........... 95

Figure 4.13 Influence of $R_{i}$ value on aortic flow and pressure..................... 96

Figure 4.14 Influence of $R_{i}$ value on accumulator flow and pressure ................ 97

Figure 4.15 Influence of $R_{i}$ value on aortic pressure (detailed comparison) ......... 98

Figure 4.16 Influence of $R_{i}$ value on LV pressure and accumulator pressure ........ 99

Figure 4.17 Influence of $R_{i}$ value on blood flow (detailed comparison)............... 99 
Figure 4.18 Influence of changing $\mathrm{R}_{\mathrm{i}}$ value on blood pressure ................... 100

Figure 4.19 Influence of changing $R_{i}$ value on blood ejection $\ldots \ldots \ldots \ldots \ldots \ldots \ldots \ldots \ldots \ldots$

Figure 4.20 Influence of changing $\mathrm{R}_{\mathrm{i}}$ value on pulse pressure .................... 101

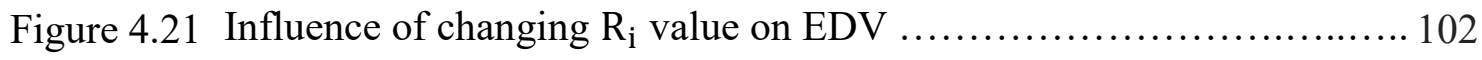

Figure 4.22 Influence of changing $R_{i}$ value on ejection phase duration ............... 104

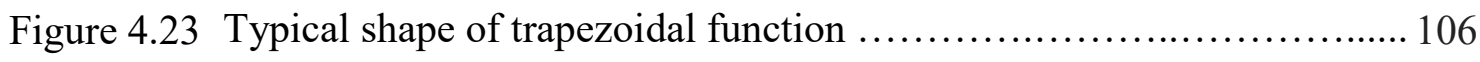

Figure 4.24 Typical shape of cycloidal function ............................. 107

Figure 4.25 Generating 4 different signals of flow rate of discharged blood ....... 109

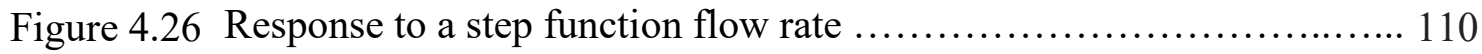

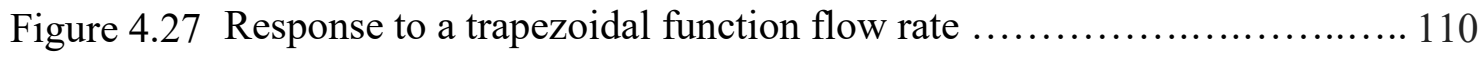

Figure 4.28 Response to a sinusoidal function flow rate ........................ 111

Figure 4.29 Response to a cycloidal function flow rate ....................... 111

\section{Chapter 5}

Figure 5.1 Implementation of tracking system optimal controller ............... 123

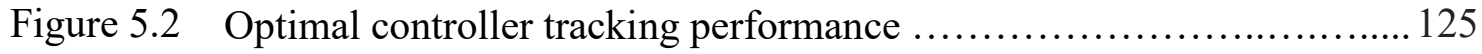

Figure 5.3 Waveform profiles of control input for trajectory tracking ............. 127 


\section{NOMENCLATURE}

AoP

AP

C

$C_{1}(t)$

$C_{2}$

$C_{3}$

$C_{4}$

$C_{5}$

$C_{6}$

$\mathrm{CCM}$

CF

$\mathrm{CO}$

$\mathrm{D}_{\mathrm{a}}$

$\mathrm{D}_{\mathrm{m}}$

$d p / d t$

$E(t)$

$\dot{E}(t)$

EDP

EDPVR

EDV

$\mathrm{E}_{\text {es }}$

EF

$\mathrm{E}_{\max }$

$E_{\min }$

ESPVR

ESV

F

FR
Aortic pressure

Arterial pressure

Capacitance, or compliance

Time-varying compliance of the left ventricle

Left atrial compliance

Arterial compliance

Aortic compliance

Artificial compliance

Accumulator capacitance

Cardiac contractility modulation

Continuous flow

Cardiac output

A diode representing the aortic valve

A diode representing the mitral valve

Pressure-rise-rate

Elastance function

Time derivative of $E(t)$

End-diastolic pressure

End-diastolic pressure-volume relationship

End-diastolic volume

End-systolic elastance

Ejection fraction

Maximum value of $E(t)$

Minimum value of $E(t)$

End-systolic pressure-volume relationship

End-systolic volume

Force applied on the accumulator's piston

Flow rate 


\begin{tabular}{ll}
$g(t)$ & $n$ vector of the optimal controller \\
$H$ & Hamiltonian function \\
$\mathrm{HF}$ & Heart failure \\
$\mathrm{HH}$ & Hodgkin-Huxley \\
$\mathrm{HR}$ & Heart rate \\
$i_{\text {accumulated }}$ & Current (blood) flowing into the accumulator \\
$i_{\text {Discharged }}$ & Current (blood) flowing out of the accumulator \\
$i_{E j e c t e d}$ & Current (blood) ejected from the LV through the aortic valve \\
$i_{R_{p}}$ & Current (blood) flowing through the peripheral resistance \\
KCL & Kirchhoff's current law \\
KVL & Kirchhoff's voltage law \\
$L$ & Inductance (blood inertance) \\
LA & Left atrium \\
LAP & Left atrial pressure \\
LQT & Linear quadratic tracking \\
LV & Left ventricle \\
LVAD & Left-ventricle assist device \\
LVP & Left-ventricular pressure \\
LVV & Left-ventricular volume \\
MAP & Mean atrial pressure \\
$P$ & Pressure \\
$P(t)$ & Riccati coefficient symmetric matrix \\
$\mathrm{P}_{\text {acc }}$ & Pressure inside accumulator \\
$\mathrm{P}_{\mathrm{d}}$ & Diastolic pressure \\
Pes & End-systolic pressure \\
$\mathrm{PP}$ & Pulse pressure \\
$\mathrm{Ps}$ & Systolic pressure \\
$\mathrm{PV}$ loop & Left-ventricular pressure-volume loop \\
$\mathrm{Q}_{\mathrm{A}}$ & Aortic flow \\
\hline &
\end{tabular}




\begin{tabular}{ll}
$Q_{a}$ & Blood flow through aortic valve \\
$Q_{i n}$ & Flow in \\
$Q_{m}$ & Blood flow through mitral valve \\
$Q_{\text {out }}$ & Flow out \\
$R$ & Resistance \\
$R_{a}$ & Aortic valve resistance \\
$R_{c}$ & Characteristic resistance \\
$\mathrm{RH}$ & Resistant hypertension \\
$R_{i}$ & Accumulator's inlet resistance \\
$R_{m}$ & Mitral valve resistance \\
$R_{o}$ & Accumulator's outlet resistance \\
$R_{p}$ & Total peripheral resistance \\
$\mathrm{SV}$ & Stroke volume \\
$t_{0}$ & Initial time of accumulator discharging \\
$\tau$ & Time constant \\
$t_{f}$ & Final time of accumulator discharging \\
$\theta$ & Angle of the rolling disk of the cycloid \\
$\mathrm{TPR}$ & Total peripheral resistance \\
$t_{r}$ & Rising time of the trapezoidal function \\
$U(t)$ & Control input (variable voltage source) \\
$v$ & Voltage \\
$V$ & Volume \\
$V_{0}$ & Theoretical value representing LVV when LVP is zero \\
$\mathrm{V}_{\text {acc }}$ & Volume of accumulated blood in accumulator \\
$\mathrm{V} \_$ejct & Volume of ejected blood through the aortic valve during the cardiac cycle \\
$\mathrm{WK}$ & Windkessel \\
$x$ & State space variable \\
$y(t)$ & System's output \\
\hline$(t)$ & Desired output \\
\hline & Co-state variable \\
\hline &
\end{tabular}




\begin{abstract}
Hypertension contributes to cardiovascular morbidity and mortality worldwide. While many hypertensive patients respond to drug therapy, a growing number of these cases are called resistant hypertension $(\mathrm{RH})$, when patients cannot control their blood pressure to goal levels despite the use of multiple antihypertensive medications.

While current interventional treatments for RH are based on dealing with the nervous system, there is no existing procedure that considers altering the way in which blood is pumped into the aorta such that to reduce blood pressure. We hypothesize that RH may be controlled by altering the way in which blood is pumped into the aorta.

We introduce a novel idea of implementing what we called the accumulator device, which may be classified as a mechanical assist device for the cardiac system. A lumpedparameter model describing the cardiovascular system is presented and validated. The novel idea of accumulator device is also modeled and incorporated with the cardiovascular system model using analogies between the circulatory system, hydraulic systems, and electric circuits.

The simulation work of the proposed accumulator device idea reveals promising preliminary results. It shows an ability to significantly decrease the systolic pressure by regulating the way in which blood is pumped into the aorta during the cardiac cycle, without reducing the cardiac output. It is our hope that this novel approach provides a transformational alternative to existing methods for treating $\mathrm{RH}$, becomes a successful treatment option in the future, and improves life for millions of RH patients.
\end{abstract}




\section{CHAPTER 1 Introduction}

\subsection{Background and Motivation}

The heart is a specialized muscle that contracts continuously and regularly. Among its four chambers, the left ventricle (LV) represents the primary pump that pumps the oxygenated blood into the systemic circulation through the aortic valve (Figure 1.1). Each time the heart beats, blood is pumped through the vascular system of the body. The measure of the force exerted by the blood against artery walls is referred to as blood pressure. Hypertension, or high blood pressure, is a long term medical condition that describes blood pressure measurements that are above normal levels.

Hypertension contributes to cardiovascular morbidity and mortality worldwide. While many hypertensive patients respond to pharmacological therapies, there is a group of patients that are resistant to such drug treatments. These patients are diagnosed as having resistant hypertension $(\mathrm{RH})$, defined more specifically as a failure to achieve target blood pressures $(<140 / 90 \mathrm{~mm} \mathrm{Hg})$ despite the use of multiple antihypertensive agents, including a diuretic [1]. It is predicted that $41.4 \%$ of US adults will have hypertension by 2030 [2]. A considerable percentage of hypertensive patients $(\sim 30 \%)$ are classified as RH patients. Studies suggest that effective treatment of hypertension reduces the incidence of heart failure and stroke by up to $50 \%$ [3].

Current methods for treating $\mathrm{RH}$ include some surgical procedures that are controversial and not without medical complications. These interventional procedures, which will be discussed in the literature review, mainly deal with the heart indirectly via 
the nervous system and do not consider altering the way the heart pumps blood into the aorta. The main objective of this research is to investigate possible alternative options for treating $\mathrm{RH}$. We hypothesize that hypertension and the pressure-rise-rate $(d P / d t)$ within the aorta can be controlled by altering the way in which blood is pumped into the aorta. This approach is totally new and represents a transformational alternative to existing methods for treating $\mathrm{RH}$.

In this research, we review the concepts of some promising device-based therapeutic technologies that have recently been widely used in treating heart failure (HF). Our investigation will check whether such technologies could also be possible candidates for treating $\mathrm{RH}$, besides their primarily effective role in treating HF. More importantly, we investigate if the concept of such device-based cardiovascular therapies could inspire us to come up with a novel approach for treating $\mathrm{RH}$.

The left-ventricular assist device (LVAD) and cardiac contractility modulation (CCM) represent two of the most promising modern device-based therapies that are used for treating $\mathrm{HF}$, a disease describing patients with a LV that is not capable of pumping the sufficient amounts of blood that their body needs. An LVAD is an implantable device with a controlled pump that pumps blood from the LV into the aorta, either by augmenting the left ventricular function or in a total capture fashion. The device was initially used as a temporary bridge to heart transplantation before it has recently been used for long-term purposes. On the other hand, $\mathrm{CCM}$ is an implantable device that can modulate the contractile force of the LV (either enhance or depress but is mainly used for enhancement purposes) via extracellular electrical stimuli. 
Such investigations require the development of a lumped-parameter reliable mathematical model for the cardiovascular system that can reproduce hemodynamics, such as blood pressure and flow, that are consistent with clinical data. After validating the cardiovascular model, we perform preliminary studies to demonstrate possible treatment opportunities for RH.

The concept of circulatory assist devices, such as the LVAD, has inspired us to propose a novel approach for treating RH. We introduce a novel idea of implementing what we called the accumulator device, which may be classified as a mechanical assist device for the cardiac system. The working concept of this device depends on accumulating an amount of blood that the LV ejects during systole. This accumulated blood is then redelivered to the arterial system during diastole such that stroke volume (SV), the amount of blood circulating through the body during one cardiac cycle, is not reduced. The paradigm for this type of pressure control comes from engineered fluid-pressure systems that are commonly used in the automotive and aerospace industries where pump control is used to limit fluid pressure $[4,5]$. We will show how the implementation of this novel approach could improve decreasing the systolic blood pressure, significantly. To the best of our knowledge, there is no existing work in the literature that investigates treating $\mathrm{RH}$ by altering the way the heart pumps blood into the aorta. It is our hope that this novel approach proves to be a successful treatment option in the future that can improve life for millions of RH patients. 


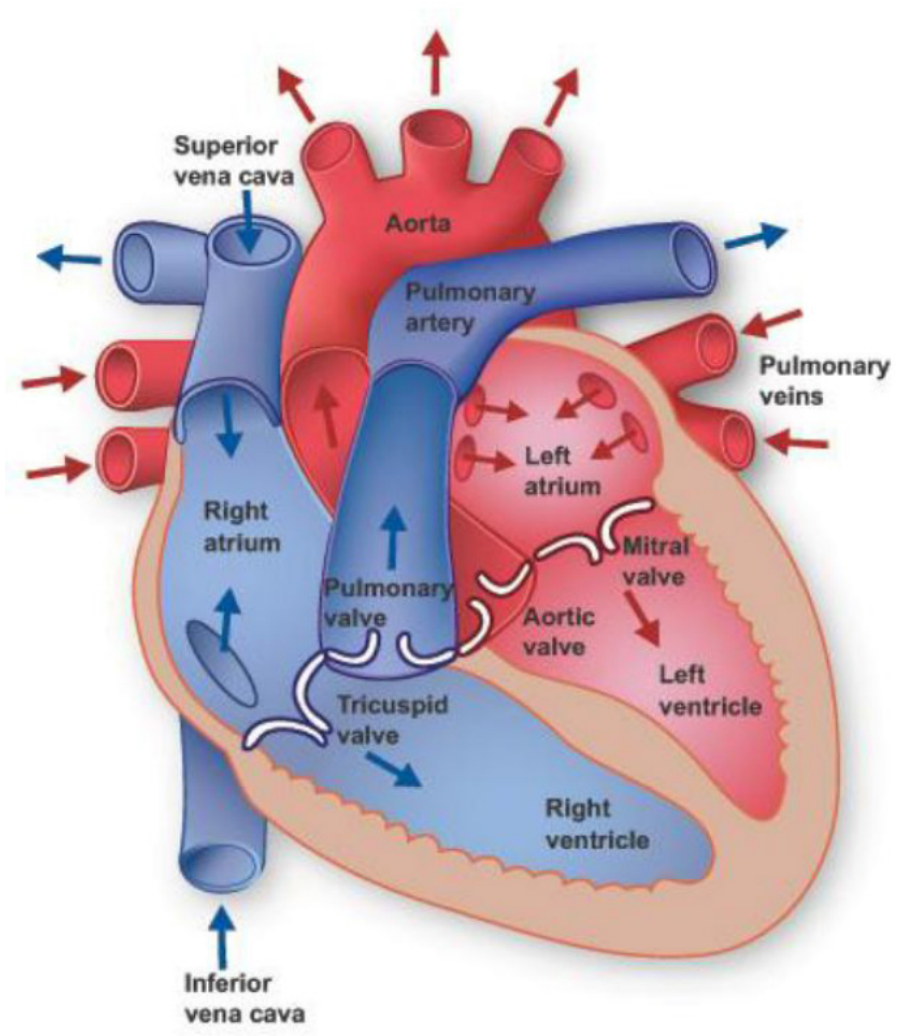

Figure 1.1. Anatomy of the heart. The figure shows the right side and left side of the heart. Each side consists of an atrium and a ventricle. The mitral valve separates the left atrium from the left ventricle and allows for unidirectional blood flow from the left atrium to the LV. The aortic valve separates the LV from the aorta and allows blood to flow from the LV into the aorta [6].

\subsection{Literature Review}

Most hypertension treatment methods are based on reducing total peripheral resistance through lifestyle changes and drug therapy. However, $\mathrm{RH}$ patients, as mentioned earlier, do not respond to such drug treatment. Current treatment methods for RH require drastic surgical procedures that primarily involve dealing with the nervous system. To the best of our knowledge, there is no existing treatment approach in the literature that treats hypertension by altering the way in which blood is pumped into the aorta. This means that 
our approach is novel and represents a transformational alternative to existing methods for treating hypertension.

We start our literature review by briefly presenting the main existing methods for treating RH. We then go over two of the most promising device-based therapeutic technologies that are used now for treating heart failure, a disease that represents one of the most common cardiovascular diseases. The core work relating to our proposed approach of the accumulator device was born after considering investigating the feasibility of implementing the concepts of such kind of devices to treat RH. After that, we present a brief glance regarding the history of the development of the mathematical models describing the heart rhythms. This kind of mathematical modeling typically aims to represent the time series of the electrical activity of the heart, called action potential (AP). We then move to a key aspect in our research which involves discussing the evolution and development of the lumped-parameter models describing the cardiovascular system. Such models represent the testbed for our modeling investigations. Finally, we present some studies that involved the use of LVADs along with their associated designed controllers in treating HF.

\subsubsection{Current Treatments for $\mathrm{RH}$}

Current treatment strategies for RH can be divided into traditional, such as lifestyle changes and escalating pharmacologic treatments, and interventional procedures [7]. The surgical treatment options include implantable stimulators for the carotid sinus baroreceptors $[3,8,9]$, renal denervation [10], and arteriovenous anastomosis $[11,12]$. 
Electrical stimulation of the carotid sinus via a surgically implantable device represents a promising therapy that produces a significant decrease in chronic blood pressure. Arterial baroreceptors, which are located in the carotid sinus and aortic arch, are mechanosensors that are activated by the pressure-induced stretch of vessel walls. Activation of these receptors results in lowering of blood pressure. Thus, the baroreceptor mechanism represents a built-in feedback control system for arterial blood pressure [13]. Studies focusing on carotid sinus nerve stimulation as a therapeutic option for hypertension treatment date back to the 1950s [8]. The early study in [9] demonstrated the effect of electrical stimulation signal intensity and concluded that as the intensity is increased, the heart rate and mean arterial pressure (MAP) decrease and that these effects were abolished upon stopping stimulation of the carotid sinus nerve.

Another surgical option for treating RH is known as renal denervation, which has been shown to be an effective surgical procedure for hypertension treatment. The sympathetic nervous system (SNS) is known to increase blood pressure via increased renal sympathetic activity. Renal denervation is a catheter-based procedure that aims to ablate the nerves in renal artery walls to disrupt the sympathetic nerve activity between the kidney and the brain. Thus, renal denervation treatment effectively lowers systolic and diastolic blood pressure in RH patients.

Arteriovenous anastomosis also represents another treatment option for $\mathrm{RH}$ patients. The procedure reduces vascular resistance and increases arterial compliance via creating anastomosis (cross connection) between the iliac artery and vein. Accordingly, the arteriovenous anastomosis surgical procedure significantly reduces both systolic and diastolic blood pressure. 


\subsubsection{Existing Device-based Therapies for Treating HF}

The artificial pacemaker, which is an implantable device that is designed to regulate the rhythmic beating of the heart, was first introduced in 1932 [14]. Although this technology is widely known to be the most commonly used device-based therapy in treating cardiovascular diseases, our mining targeted two other technologies that represent modern treatment options in treating heart failure. In this section, we review the leftventricular assist device (LVAD) and cardiac contractility modulation (CCM) device.

\subsubsection{Left-ventricular Assist Device (LVAD)}

An LVAD is an implantable mechanical pump developed to support a failing LV by supplying the body with additional blood to restore normal hemodynamics [15]. It directs blood from the LV into the aorta, and so it typically has an inlet that is connected to the LV and an outlet that is connected to the aorta, making it work in parallel with the LV. The LVAD was initially proposed for short-term use as a temporary bridge to heart transplantation in HF patients. The prevalence of heart failure and lack of donor hearts necessitated the development of mechanical assistance devices such as the LVAD. Technological advancements have significantly improved LVADs now, making them an increasingly prevalent therapy for long-term use (destination therapy) as an alternative to a heart transplant [16]. A typical implanted LVAD and its components are shown in Figure 1.2. 


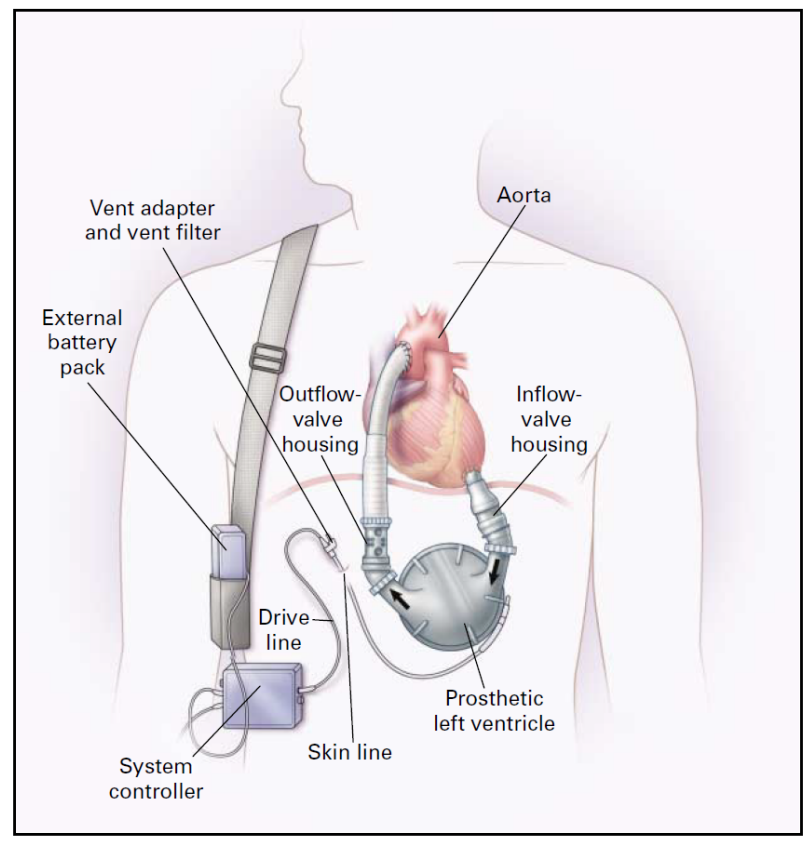

Figure 1.2. Components of a typical LVAD. The inflow cannula is the inlet of the LVAD that is connected to the LV, and the outflow cannula represents the LVAD outlet that connects to the aorta. Blood exits the LV into the LVAD pumping chamber through an inlet valve before it is pumped out through the outlet valve into the aorta [17].

Various circulatory-assist devices have been developed since 1964, when the National Heart, Lung, and Blood Institute (NHLBI) established the artificial-heart program [18]. In 1994, the FDA (Food and Drug Administration) approved the first use of an LVAD as a bridge to transplantation [15]. Pulsatile volume displacement pumps represent the first generation of LVADs. While pulsatile LVADs are believed to be more physiologic due to their pulsatility, this generation of LVADs was not widely used due to their large pump size, limited durability, the need for inflow and outflow valves, and the complication of their control strategy [19]. Inventors began developing smaller, continuous-flow LVADs in the early 1990s to reduce complications that resulted from the use of the first-generation pulsatile LVADs [20]. Continuous-flow (CF), non-pulsatile LVAD technology has rapidly developed during the past couple of decades due to its durability and smaller size. The 
second and third generations of LVADs are valveless, continuous-flow pumps; while the former generation utilizes axial pumps, the latter utilizes centrifugal pumps [16]. Figure 1.3 presents a schematic for a continuous-flow LVAD system and its different pump types.

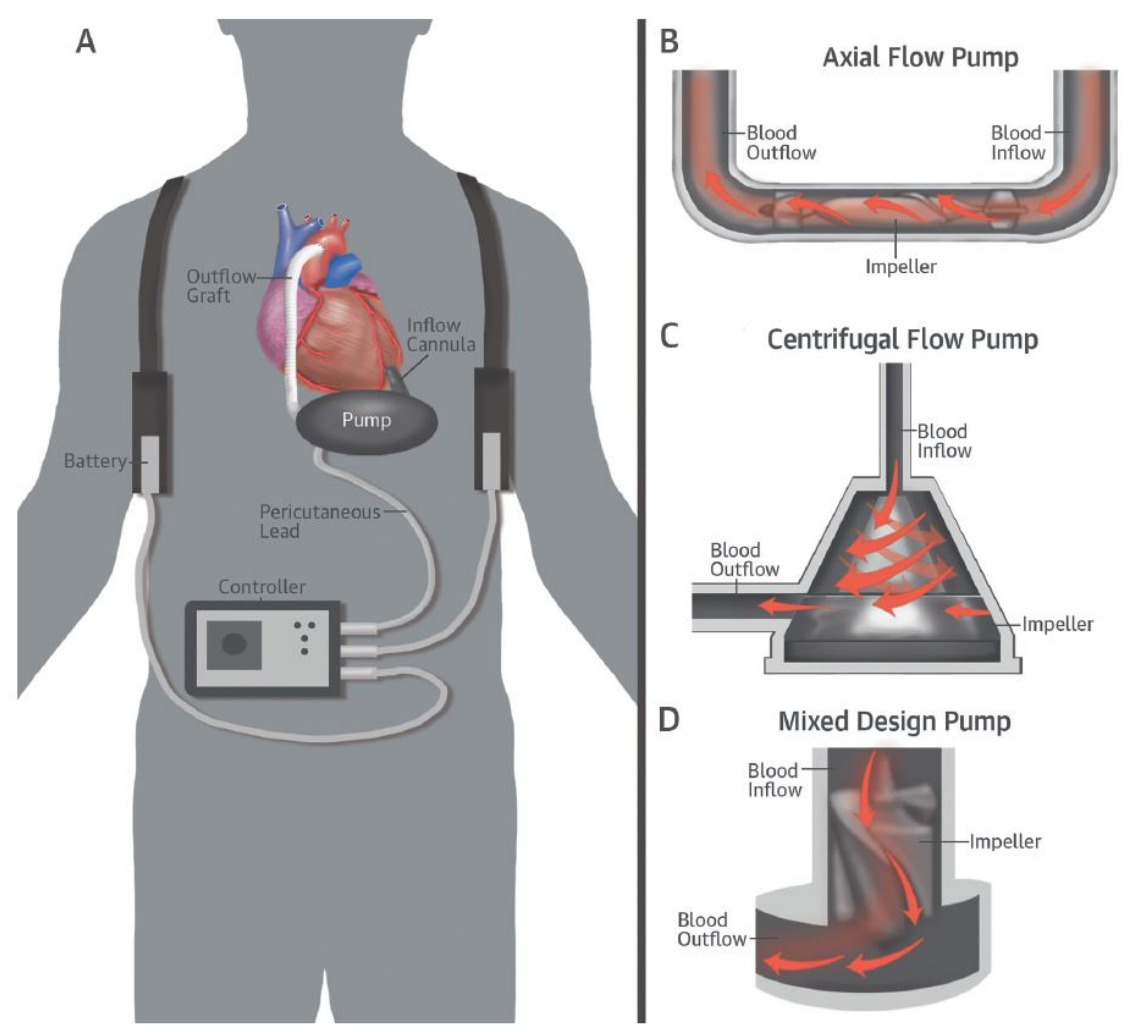

Figure 1.3. Components of an LVAD system are shown in (A). Continuous-flow axial type pump shown in (B), a centrifugal type pump is shown in (C), and mixed design pump shown in (D) [16].

The improved durability of the rotary pumps implemented in the second and third generations of LVADs is associated with a compromise of having a pulseless, continuous blood flow that runs at a constant speed. The presence of pulseless, nonphysiologic blood flow and the likelihood of aortic valve permanent closure associated with the long-term use of CF LVADs cause a decrease in arterial pulsatility, which in turn leads to critical medical complications. Arterial pulsatility is characterized by pulse pressure (PP), which is defined as the pressure difference between systolic and diastolic pressure measurements. 
This observation led to an interest in developing control algorithms that would allow for generating pulse pressure in CF LVADs in an attempt to decrease the complications associated with the diminished pulsatility resulting from the long-term use of CF LVADs [21]. Recent studies aim to develop methods for generating more pulsatility and avoiding aortic valve permanent closure. This can be achieved by designing controllers that modulate the speed of the pump in order to generate intrinsic pulsatile flow from the LVAD itself and/or can allow the native heart to periodically contract and eject blood on its own [16]. The study in [22] showed that it is possible to generate a pulsatile pressure close to that of the native heart using a CF pump LVAD.

Since 2012, the number of implanted LVADs as a destination therapy has exceeded the number of those implanted temporarily as a bridge to heart transplant [16]. This indicates that the rapid improvement of LVAD technology has allowed it to become the most commonly used long-term myocardial therapy.

\subsubsection{Cardiac Contractility Modulation (CCM)}

Cardiac contractility modulation represents another relatively new therapeutic technology that effectively treats HF patients, primarily those with reduced ejection fraction (EF) (a measurement describing the ratio between the amount of ejected blood and total blood volume in the LV within one cardiac cycle) [23]. CCM is an implantable, pacemaker-like device that can modulate the cardiac contractility by applying electric signals to improve the cardiac pump function. The signals are delivered during the absolute refractory period of the cardiac AP; hence they are called non-excitatory signals. A typical protocol for CCM signals application is shown in Figure 1.4. 
CCM signals are relatively high-voltage, bi-phasic, square wave signals. They are delivered to the cardiac muscle about $30-40 \mathrm{~ms}$ after local electrical activation, which is detected by sensing leads. This delay in delivering CCM signals is to ensure that they are applied during the absolute refractory period so that they do not induce additional action potentials or cause contractions (therefore resulting in no change in the heart rhythm) $[24$, 25]. A typical CCM device mainly consists of a pulse generator and electrodes that sense local electrical activation and deliver CCM signals [25].

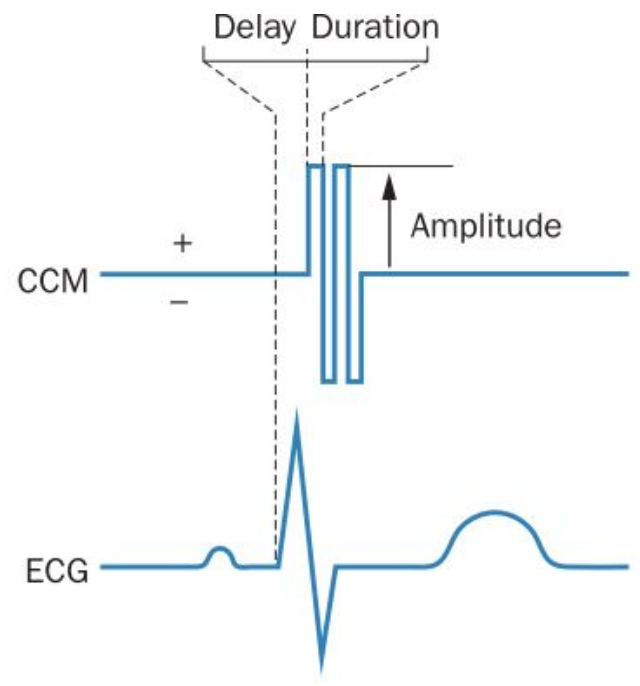

Figure 1.4. CCM signals application. Local electrical activation is detected by sensing leads as shown in the lower panel. Upper panel shows the delay of CCM delivery after detection of local activation to ensure that signals are delivered during absolute refractory period [24].

Calcium represents the most important ion underlying the heart working process as it enables the heart muscle to contract and relax [26]. It was in the early 1960s when calcium was found to be the main physiological activator of the contractility and since then, it has become the key to understanding contractility [27]. Early studies showed that calcium entry and contractility could be enhanced by modulating the action potential (AP), the 
electrical signal that activates the heart muscle to contract [28]. CCM signals application represents a conceptual breakthrough since it influences calcium and contractility via field stimulation of the cardiac muscle [29]. The initial study in [30] demonstrated that applying nonexcitatory electric currents (CCM signals) to the LV enhanced its performance by inducing an increase of about $10 \%$ in maximum $d P / d t$ and aortic pulse pressure. The study in [31] investigated how CCM signals modulate cardiac contractility. They reported that CCM signals increased mean arterial pressure but did not affect the peripheral resistance. They showed that the global contractility index $\mathrm{E}_{\text {es }}$ (end systolic elastance) was increased by $\sim 30 \%$ and $\sim 70 \%$ and that maximum pressure-rise-rate $d P / d t$ was increased by $\sim 25 \%$ and $\sim 30 \%$ with single-site and dual-site stimulations, respectively. They concluded that all parameters return to baseline conditions within 1 min after stopping the application of CCM signals.

The study in [32] showed that applying CCM signals significantly influenced the contractile force. They stated that negative amplitude (cathodic) current signals reduced the contractility force whereas positive amplitude (anodic) current signals increased it. They showed that the effect of CCM signals of either increasing or decreasing the contractility force was instant and that contractility was modified within just one beat after applying the signals and reached a new steady-state level after 6-8 beats. Brunckhorst et al [33] showed that the modulation that CCM signals caused to AP duration (shortening or lengthening) and contractility (enhancement or depression) were dependent on the amplitude, duration, and delay of the applied electric signals. Their study was exclusively focused on positive CCM signals (enhancing the contractility) since the primary goal of such studies is for treating HF patients to improve their ejection fraction (EF); however, 
they clearly indicated that the negative inotropic effect, i.e. decreased contractility, still holds with the application of negative CCM signals. Figure 1.5 illustrates the results of their study regarding the opposite effects of positive (anodic) and negative (cathodic) CCM signals on contractility. They concluded that CCM therapy gives the same results of enhancing (or depressing) the ventricular contractility as that seen in the earlier voltage clamping technique; however, unlike the voltage clamping method which is not applicable to the intact heart, CCM can be applied safely to both normal and failing hearts. This makes CCM a promising new form of electrical treatment.

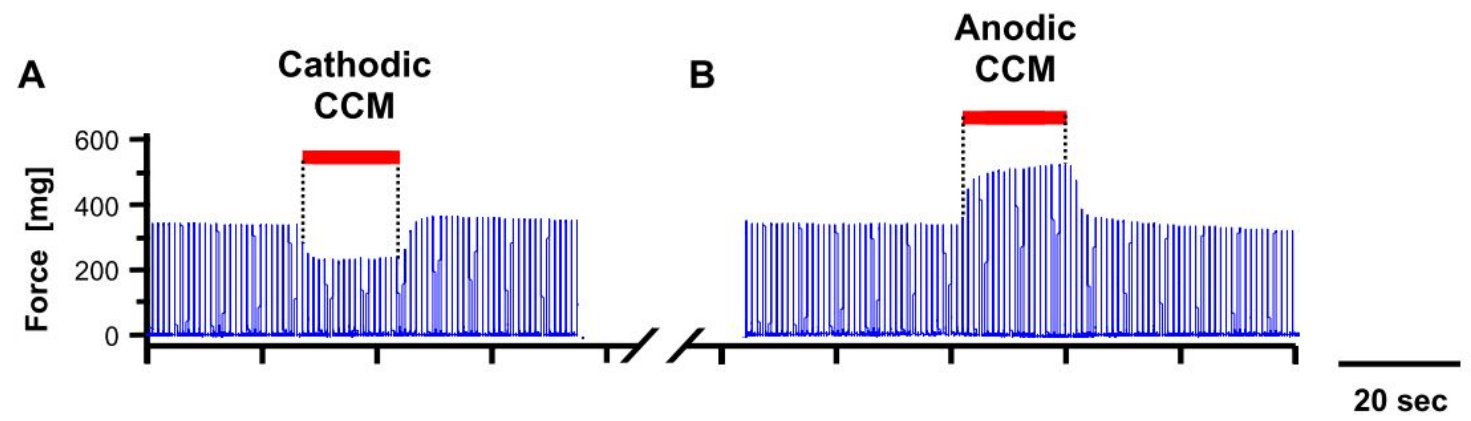

Figure 1.5. Effects of positive and negative CCM stimuli on contractility. Negative (cathodic) signals (A) decrease contractile force while positive (anodic) signals (B) increase contractility [33].

\subsubsection{Mathematical Models for Heart Rhythms}

Mathematical models describing heart rhythm dynamics are widely used and date back to the 1920s when the classic Van der Pol oscillator [34] was first introduced. These models are mainly aimed at representing the time series of the cardiac AP, a phenomenon that describes the electrical activity of the heart, to study some important physiological features of the cardiac AP such as firing frequency, refraction time, etc. A brief history of the development of such models will be discussed next. 
The nonlinear relaxation oscillator, introduced by the Dutch electrical engineer and physicist Van der Pol, represents the starting point for modeling the rhythmic behavior of the heart. The oscillator, which is described in equation (1.1), has shown to be very useful in modeling the heartbeat since it can perfectly demonstrate important features such as synchronization and limit cycles.

$$
\ddot{v}-\alpha\left(1-v^{2}\right) \dot{v}+\omega^{2} v=0
$$

Alan Hodgkin \& Andrew Huxley (1952) presented a mathematical model that perfectly predicted the shape of the AP [35]. Their model, which is known as the HodgkinHuxley $(\mathrm{HH})$ equation, was based on giving mathematical reconstructions for the kinetics of the ionic channels, such as sodium and potassium, that underlie the depolarization and repolarization processes. Their fascinating work on this model was the reason they received the Nobel Prize for Physiology and Medicine in 1963. Fitzhugh [36] proposed a model that represents an extension to the Van der Pol's equation [34] and a simplification of the $\mathrm{HH}$ model [35] by using two variables instead of four as in the HH model. The resulting model has been shown to clearly exhibit basic dynamics such as threshold and refractoriness but was not intended to be accurate in reproducing the shape of experimental curves.

Abrams et al [37] used a modified formulation for the Van der Pol oscillator to describe the ventricular pressure-volume relationship. The Van der Pol's formulation used in this study is shown in equation (1.2) where $P$ and $V$ represent the left ventricular pressure and volume, respectively, whereas $\mu$ and $\sigma$ represent phenomenological parameters that describe the systolic and diastolic functions; respectively. 


$$
\left\{\begin{array}{l}
\frac{d P}{d t}=\mu\left[V-\left(\frac{P^{3}}{3}-\alpha P\right)\right] \\
\frac{d V}{d t}=-\frac{P}{\mu}
\end{array}\right.
$$

The use of modified Van der Pol's equations in (1.2) facilitated the process of reconstructing the left ventricular pressure-volume loops from the continuous measurement of cardiac output (CO) and left ventricular pressure. The study in [38] presented a modified model for the Van der Pol oscillator that can reproduce the time series behavior of the cardiac AP. They stated that the classic Van der Pol equation in [34] and other models were interesting models because they represented the physical phenomena of heart rhythms; however, these models failed to simulate many important physiological features. For instance, they mentioned that changing $\alpha$ in the classic Van der Pol's equation in (1.1) affects both refraction and diastolic periods. This is not desirable since such models should offer the possibility of changing refraction and spontaneous depolarization (diastolic) time, independently. Based on this, they modified the phase space of the classic Van der Pol equation and came up with a modified model that can reproduce the experimental results while allowing to independently manipulate the diastolic and refractory periods, which represent two important physiological quantities.

\subsubsection{Lumped-parameter Models: the Windkessel (WK) Models}

The cardiovascular system is a closed-loop, dynamic system that is driven by the heart. Modeling the cardiovascular system represents a helpful tool for obtaining some knowledge about the hemodynamics of the arterial system, such as blood pressure and 
flow, that can facilitate further physiological studies of the cardiovascular system. Lumped parameter models, unlike higher dimensional models, assume a uniform distribution of variables, such as pressure, within any specific compartment, such as a vessel, at any instant of time [39]. Windkessel (WK) models are lumped-parameter models that describe the hemodynamic elements of the arterial system and are considered a frontline modeling technique for estimating key hemodynamic aspects of the arterial system [40, 41]. The basic idea of Windkessel models centers around representing the characteristic functions of the arterial system by their equivalent electrical elements.

Otto Frank, the German physiologist, introduced the first mathematical WK model in 1899 in his two-element Windkessel model [42]. In its simplest configuration, the twoelement WK model consists of two parallel elements: a resistance, called total peripheral resistance (TPR), which represents the resistance to blood flow in the arterial system by means of the smaller vessels and arterioles and a capacitance or compliance, which describes the storage properties of large vessels and their ability to accumulate and release blood due to elastic deformation. The two-element WK model shows a decent prediction of the aortic pressure during diastole, which follows a first-order exponential decay with a time constant being determined by the multiplication of the vascular resistance $\mathrm{R}$ and arterial capacitance $\mathrm{C}$; however, the systolic pressure is poorly predicted in the two-element WK model [40].

The weakness in predicting systolic pressure in the two-element WK model encouraged a number of researchers to improve it. The addition of a third element was introduced in [43]. The newly introduced element, which is called the aortic characteristic impedance, accounts for the local compliance and inertia of the proximal ascending aorta. 
The 3-element WK model, which consists of the characteristic impedance connected in series with the RC two-element WK model, shows a significant improvement in accurately predicting both systolic and diastolic aortic pressures as well as stroke volume (SV) [44]. The introduction of the characteristic impedance has greatly improved the high frequency performance that is associated with pressure reflections [45]. Although the 3-element WK model is considered the lumped-parameter model that is most widely used and accepted for describing the cardiovascular circulatory system [40], it still shows some limitations in terms of underestimating peak aortic flow and mean arterial pressure, in addition to its inaccuracy in providing realistic aortic flow and pressure when compared to other realistic models [44]. The introduction of the characteristic impedance in the 3-element WK model showed to considerably improve the medium-to-high frequency performance of the model, which was a weakness in the 2-element WK model; however, its limitation shines at low frequencies.

A fourth element, modeled as an inertance or inductance, was introduced to account for blood inertia. The addition of the inertial term contributes to the low frequencies only, while the characteristic impedance of the 3-element WK model comes into play at the medium-to-high frequencies. Thus, the inertial element compensates the inconsistency resulting from approximating the characteristic impedance as a resistance in the 3-element WK model. The 4-element WK model is the most comprehensive WK model that offers excellent wave shapes for both flow and pressure [46].

Despite their limitations, WK models remain the most commonly used and accepted lumped-parameter models for describing the cardiovascular circulatory system. Compared to other types of models, they require less computational power and thus can 
perform faster [47]. They have also shown to be useful for modeling studies that involve the insertion LVADs [41]. Figure 1.6 demonstrates the 2-element, 3-element, and 4element Windkessel models.

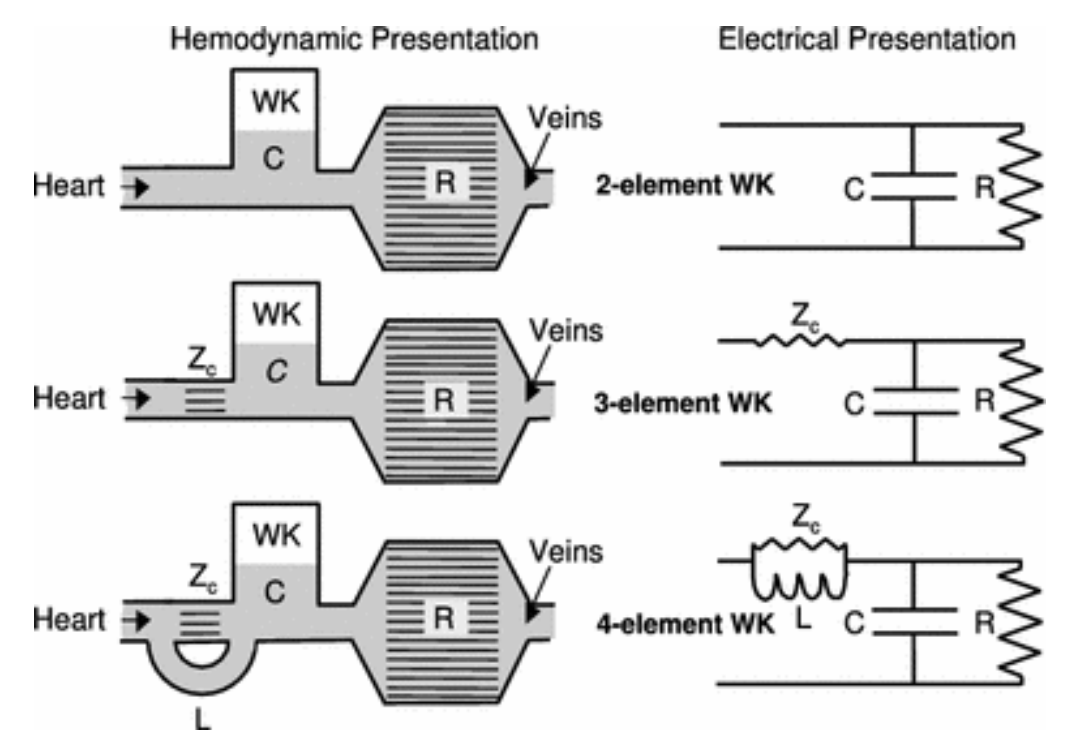

Figure 1.6. The 2-element, 3-element, and 4-element Windkessel models illustrated in hydraulic (left) and their equivalent electrical (right) configurations. $\mathrm{C}$ and $\mathrm{R}$ represent arterial compliance and vascular resistance, while $Z_{c}$ and $L$ represent the aortic characteristic impedance and inertia [40].

\subsubsection{Modeling and Control of an LVAD}

The long-term use of an LVAD requires the implementation of a controller that can automatically adjust the LVAD's pump speed. The study in [48] discussed the engineering challenge related to designing appropriate controllers for LVADs and stated that such controllers must satisfy two important criteria. First, it should automatically adapt to the patient's physiological changes by regulating the speed of the LVAD pump in such a way to meet desired pressure and cardiac output. Second, it should avoid suction of the LV, a condition that occurs when the pump is attempting to draw more blood than what is 
available from the LV. A brief overview of some LVAD control techniques developed in the past will be discussed next.

A feedback control approach to control the speed of a continuous-flow rotary pump LVAD was reported in [49]. The control strategy in this study was mainly aimed at determining the target pump speed that can provide the body with the optimum flow while preventing regurgitation and suction in the ventricle, which represent abnormal circulatory conditions. For pump speed adjustment, they utilized a predetermined relationship between the native heart rate, which is monitored continuously, and cardiac output. The target pump speed can then be derived according to another relationship between pump flow and speed. For preventing the occurrence of abnormal conditions, such as regurgitation and suction of the LV, they analyzed the motor current waveform using fast Fourier transform. A distortion in the motor current waveform is an indicator of abnormal condition occurrence. This was quantified by computing the waveform deformation index (WDI) for the waveform of the motor current. They stated that for normal operation, the WDI should be below 0.2 (a threshold value, based on a theoretical study), otherwise abnormalities exist. They reported that the WDI values above the threshold level corresponded to regurgitation when at low pump speeds and to suction when at high pump speeds.

The interaction of an LVAD, represented by a rotary-type pump, with the LV of the heart was studied in [50]. A coupled cardiovascular-pump model was presented, which utilized a lumped-parameter electric circuit model with preload being represented by a single compliance, the LV represented by a time-varying compliance, and afterload being represented by a 4-element WK model. The resulting state-space model is 5-dimensional with the five state variables representing pump flow, total blood flow through the aorta, as 
well as pressure values in the left atrium, left ventricle, and arteries. They developed a feedback controller that automatically adjusts the pump speed in order to meet the patient's physiological needs of blood while insuring the avoidance of suction. The controller was designed to increase the pump speed up to the point before suction occurrence, when at that time, it will maintain a constant pump speed.

The study in [51] presented a combined six-dimensional cardiovascular-LVAD model that is based on the model in [50] with the addition of a sixth state variable representing the pressure in the aorta (while that arterial pressure still represents another state). They developed a feedback controller that automatically adjusts the pump speed depending on the measurements sensed from the pump flow, which represents the only sensible signal out of the six state variables. Their developed controller also accounts for avoiding the occurrence of suction. The basic functions of the developed controller are tracking the pump flow signal to detect the minimum value during each cardiac cycle and then estimating the slope of the envelope of the detected minimum values. The controller then adjusts the speed of the pump based on the estimated slope in order to achieve optimum pump speed while avoiding the occurrence of suction. The controller performed very well in the simulations that involved both constant and changing systemic vascular resistance, which represent cases that emulate a patient's different levels of activities. However, in the presence of a considerable amount of noise associated with the measured pump flow signal, the controller did not perform well where pump speed did not consistently avoid the suction zone. 


\subsubsection{Literature Search Conclusion}

The literature search revealed that there is no existing work that relates to treating RH by altering the way the LV pumps blood into the aorta. The LVAD has been shown to represent a promising mechanical assist device in treating HF patients. Although we anticipate that the LVAD concept could be utilized in treating high blood pressure, since it comprises a controlled pump that can regulate the blood flow and pressure, LVADs are mainly aimed to assist failing hearts by taking over the entire pumping action, which may lead to a permanent closure in the aortic valve. Thus, it may not be desirable to use an LVAD with the intact heart of $\mathrm{RH}$ patients to treat hypertension.

The LVAD concept, however, inspired us to further examine a novel approach that relates to accumulating an amount of blood via the use of an accumulator device that is connected to the heart and aorta in a similar way to that in an LVAD. From our perspective, this approach is preferred over the LVAD for treating RH because it allows the intact heart to eject blood naturally through the aortic valve; it does so with the least interference (assistance) needed from the accumulator device to accumulate blood in order to provide the necessary relief to normalize the systolic pressure below maximum allowed levels.

Windkessel models have proven to be very useful lumped-parameter models in representing the arterial network of the cardiovascular system. The modeling research in literature that dealt with coupling an LVAD with the cardiovascular system would facilitate the implementation of the accumulator device proposed idea. The modeling strategy used in literature of the LVAD coupled with the cardiovascular system will be mimicked to perform the same with the introduced accumulator device concept. 


\subsection{Dissertation Outline}

The research in this document is organized according to the following order:

- Chapter 1 provides a background for the problem relating to investigating a new approach for treating resistant hypertension. A relevant literature review is also provided.

- Chapter 2 describes the cardiovascular system to prepare for the modeling work that will be presented in the proceeding chapters.

- Chapter 3 presents a lumped-parameter model of the cardiovascular system. The model uses some analogies between the circulatory system and electric circuit theory. The model is then validated by reproducing hemodynamics that are consistent with clinical data. Preliminary studies demonstrating possible opportunities to treat $\mathrm{RH}$ are also presented in this chapter.

- Chapter 4 introduces a new idea that represents a novel approach for treating resistant hypertension. The proposed accumulator device is implemented and incorporated with the lumped-parameter model of the cardiovascular system presented in chapter 3. The chapter then presents the results of this modeling work, which support the hypothesis that RH can be treated if we can alter the way in which the heart pumps blood into the aorta.

- Chapter 5 provides a control design for the accumulator device. Optimal control theory, specifically linear quadratic tracking, is used to design a controller such that the accumulator discharges blood according to a desired trajectory of flow rate.

- Chapter 6 lists conclusions and recommendations for future research. 


\section{CHAPTER 2 Description of the Cardiovascular System}

The heart is a muscular pump that is connected to the systemic and pulmonary vascular systems. It works together with the vasculature to supply the body with the necessary nutrients in the form of oxygenated blood that circulates throughout the body. To model of dynamics of the cardiovascular system, one needs to understand the cardiac cycle and the physiological changes that occur during it. This should also be paired with the understanding of the relevant hemodynamic changes, such as blood pressure and flow. This chapter will provide a brief overview of the cardiovascular system in preparation for the modeling work that will be described in the proceeding chapters.

\subsection{Anatomy of the Heart}

A normal heart consists of two sides, a right one and left one, with each side comprising two chambers as shown in Figure 1.1. Each side includes an atrium and a ventricle. The primary role of each atrium is receiving and accumulating the blood returning from its respective systemic circulation before pumping it to the ventricles. On the other hand, the ventricles are considered the primary pumps that circulate blood throughout the body's circulatory systems.

The right heart, consisting of the right atrium and the right ventricle, receives the de-oxygenated blood and pumps it to the lungs (the pulmonary system). The oxygenated blood returning from the lungs flows into the left atrium before it is pumped into the left ventricle across the mitral valve. The left ventricle (LV) then pumps the oxygenated blood 
into the circulatory vascular system through the aortic valve. Thus, the heart has two separate pumps: a right ventricle which pumps de-oxygenated blood to the pulmonary system and a left ventricle that pumps oxygenated blood through the body's vascular system as shown in Figure 2.1.
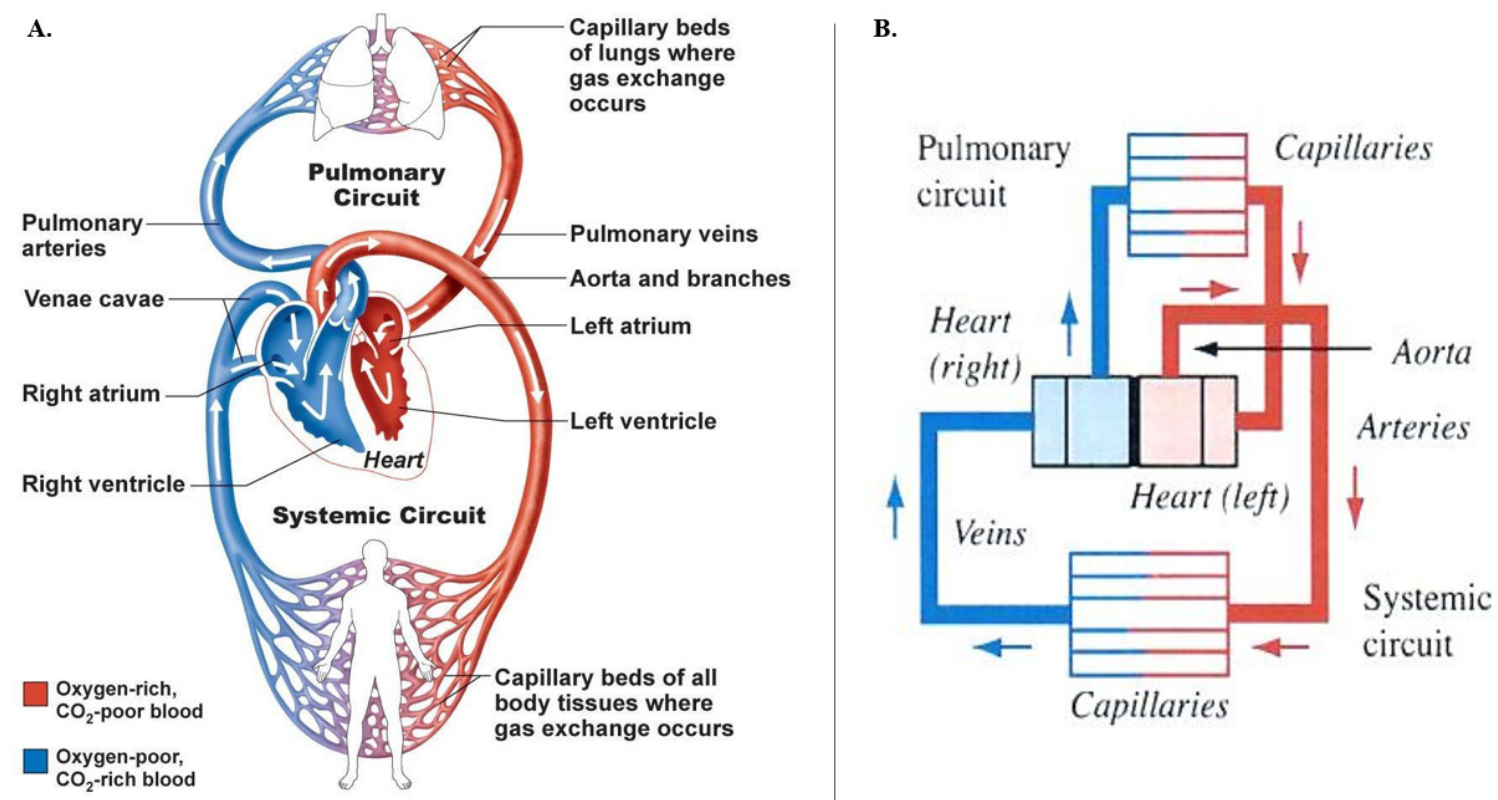

Figure 2.1. The Circulatory Loop: (A) The cardiovascular system is a closed-loop system that consists of two main fluid pumps and a network of vascular tubes. The loop is divided into the pulmonary vascular system (whose pump is the right ventricle which pumps blood throughout the lungs) and the systemic vascular system (with the left ventricle being its pump which pumps blood throughout the body). The diagram is copied from https://humananatomywiki.com/ circulatory-system-chart/circulatory-system-chart-humancirculatory-system-diagram-labeled-anatomy-chart-body/. (B) A simplified diagram for the circulatory loop of the cardiovascular system [52]. 


\subsection{Cardiac Cycle Phases}

The cardiac cycle is the event occurring from the beginning of one heart beat to the beginning of the next one. It has two main phases called systole and diastole. Systole, which means contraction in Greek, represents the period of time during which the heart muscle contracts and ejects blood from the LV into the aorta. Diastole, which means dilation in Greek, represents the period of time when the heart muscle relaxes, and the LV fills with blood. Figure 2.2 shows the effect of blood movement on artery walls when flowing into (systole) and out of (diastole) the arteries. Figure 2.3 shows the behavior of the left ventricle, aortic valve, and aorta during ventricular contraction (systole) and relaxation (diastole).

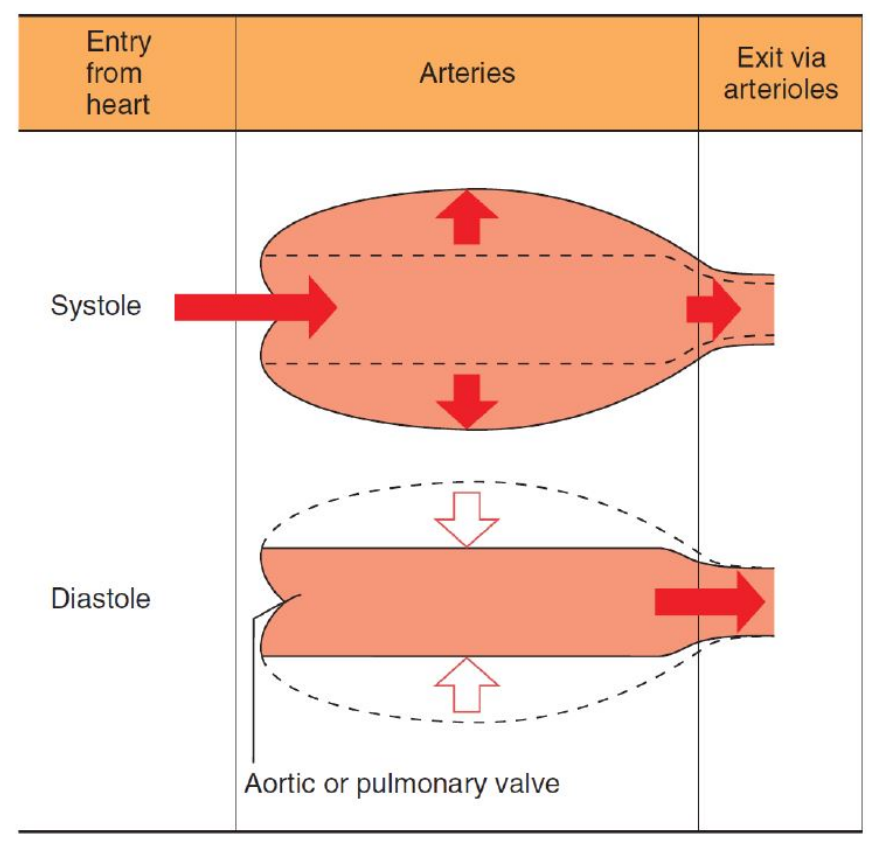

Figure 2.2. Movement of blood into and out of arteries during systole and diastole [53]. 


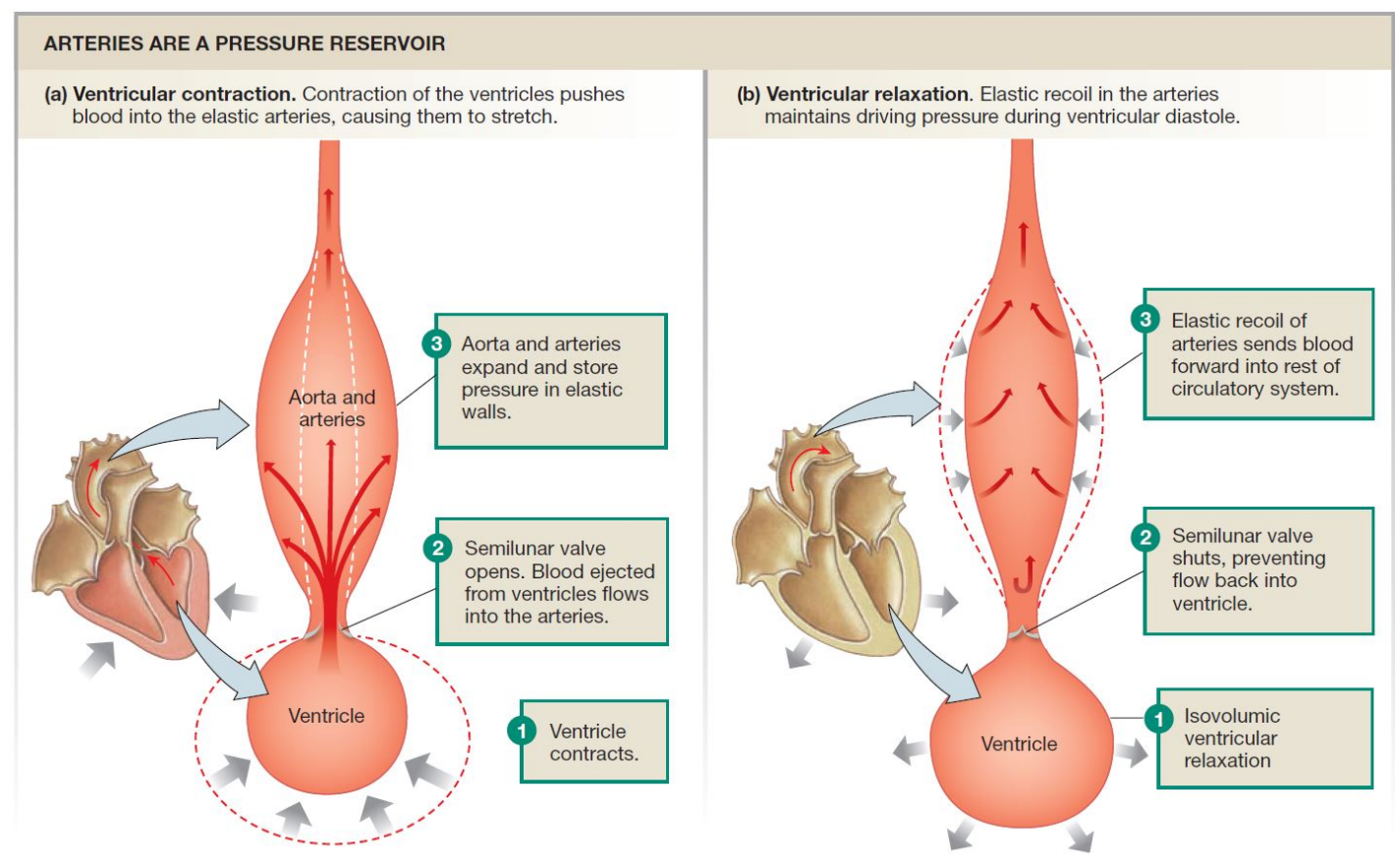

Figure 2.3 Dynamic behaviors during ventricular contraction and relaxation [53].

During each cardiac cycle, the heart goes through four phases: a phase of isovolumic contraction and an ejection phase, together representing systole, and an isovolumic relaxation phase and a filling phase, together representing diastole. The four phases are detailed more as follows:

1- Isovolumic contraction phase: This phase starts when the mitral valve, the interconnection between the left atrium and the left ventricle, closes. This closure occurs when the pressure inside the left ventricle becomes higher than the pressure in the left atrium, which is a consequence of filling the left ventricle with an adequate amount of blood. The pressure inside the LV will build up very rapidly during this phase since both the mitral valve and aortic valve are closed. This phase will end the moment the pressure inside the LV exceeds the aortic pressure. 
2- Ejection phase: The onset of this phase is determined by the time the aortic valve opens, allowing blood to flow from the left ventricle into the aorta. Blood ejection continues as long as a positive pressure gradient is held between the LV and the aorta. The mitral valve is still closed during this phase. The ejection phase ends at the time of aortic valve closure when the pressure inside the LV falls below that in the aorta.

3- Isovolumic relaxation phase: This phase represents the onset of diastole, which follows the end of the systolic phase. Both the mitral and aortic valves are closed during this phase. The pressure inside the LV will drop rapidly to very low levels to prepare for the filling phase that starts upon the mitral valve opening.

4- Filling phase: The LV starts to fill with blood when the mitral valve opens, a consequence of having a positive pressure gradient between the left atrium and the left ventricle. The aortic valve is closed during this phase, and blood will fill up the LV until the pressure inside the LV becomes higher than the pressure in the left atrium, at which time the mitral valve closes to prepare for starting the systolic phase of the next cardiac cycle, and so on.

Figure 2.4 shows a schematic that describes the above described four phases of the cardiac cycle. Figure 2.5 shows the Wiggers diagram, named after Dr. Carl J. Wiggers, which is considered the standard diagram used in cardiac physiology. The Wiggers diagram is very informative and can demonstrate the pressure changes in the left ventricle, left atrium, and aorta during each phase in the cardiac cycle. It also shows the corresponding change in the left ventricular volume, as well as the moments when the mitral valve and aortic valve open and close. 


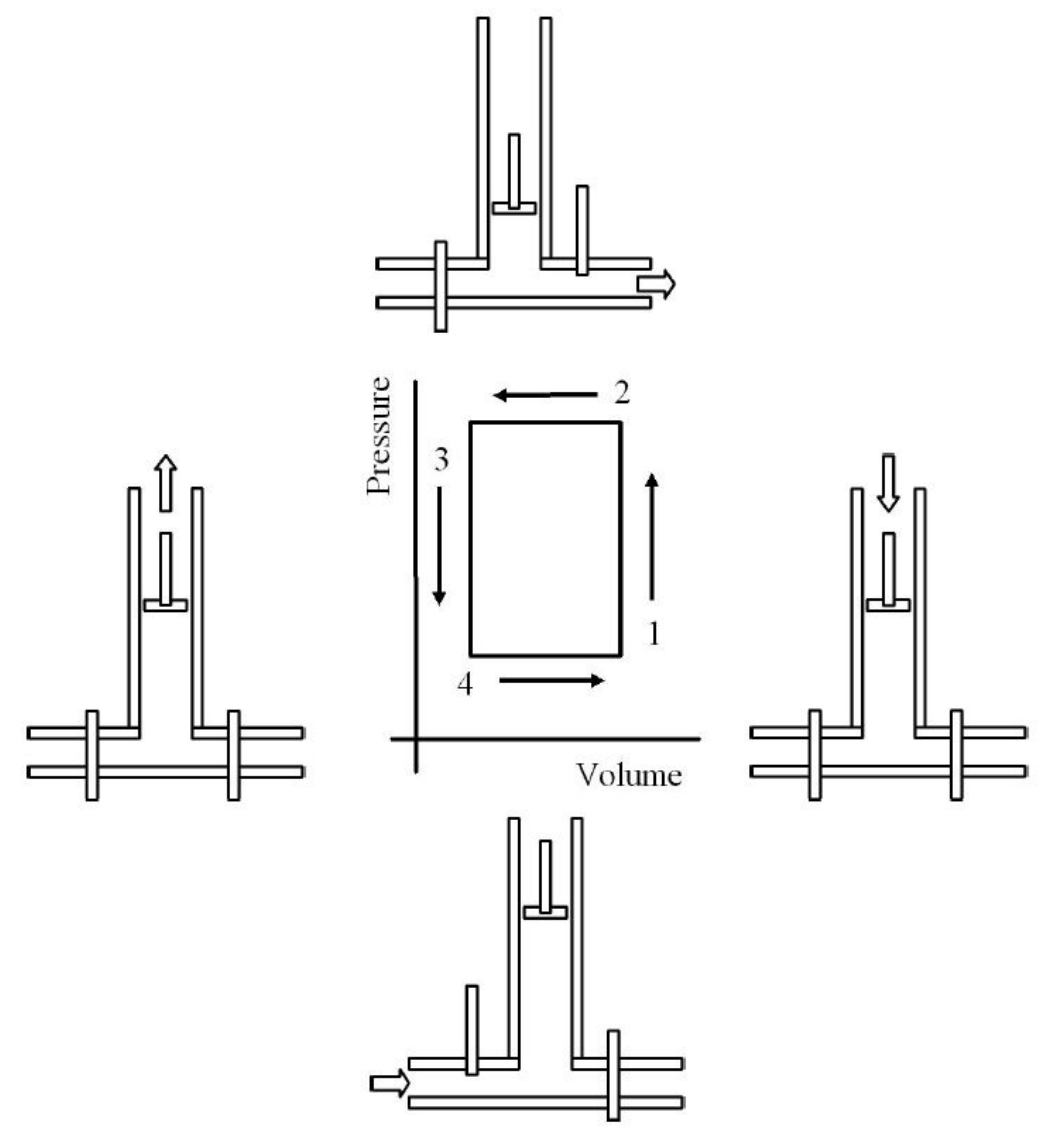

Figure 2.4. Cardiac cycle phases. Isovolumic contraction phase is shown in the schematic in the right (corresponding to the arrow labeled "1" on the plot located at the center, which is called the PV loop as will be shown later). Both mitral valve (left) and aortic valve (right) are closed, and the LV develops pressure due to contraction, represented by the downward movement of the piston. The ejection phase is shown in the upper schematic (which corresponds to label " 2 " on the plot), the aortic valve (right) opens which allows blood to flow out of the LV chamber into the aorta while the mitral valve (left) is still closed. The left schematic represents the isovolumic relaxation phase, which corresponds to label " 3 " on the plot, where both the mitral and aortic valves are closed. The pressure within the LV drops rapidly during this phase; this is represented by the piston moving upward. The lower schematic describes the filling phase, which corresponds to label "4" on the plot at the center, where the LV is filled with blood through the mitral valve which opens during this phase while the aortic valve remains closed [54]. 


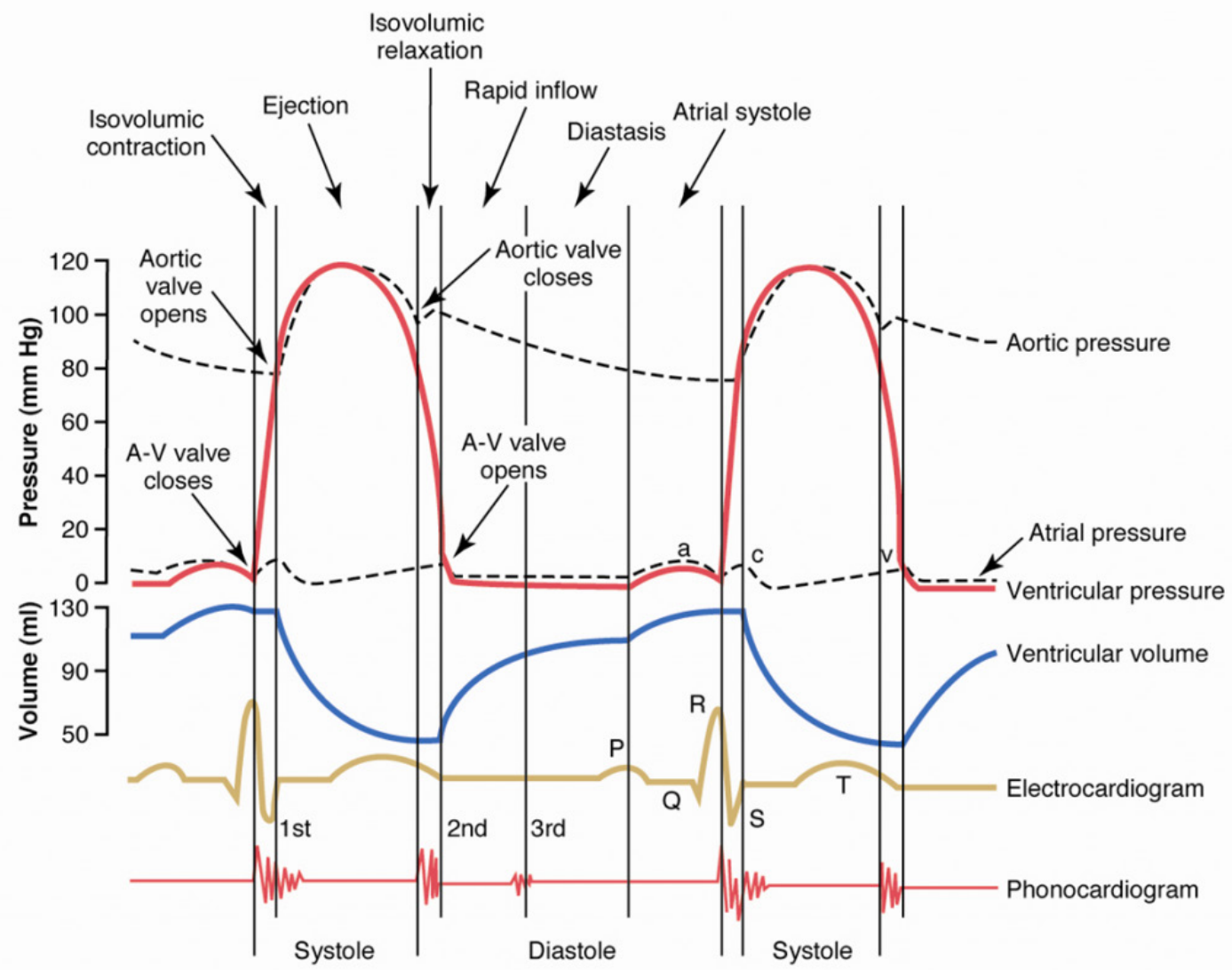

Figure 2.5. Wiggers diagram. Hemodynamic changes during the four phases (isovolumic contraction, ejection, isovolumic relaxation, and filling). Changes in pressures of the left ventricle, left atrium, and aorta during the cardiac cycle is shown in the upper plot. The corresponding change in the left ventricular volume is shown in the lower plot (figure was copied from http://www.printablediagram.com/wpcontent/uploads/2014/11/wiggers-diagram-cardiac-circle-1024x833.png).

\subsection{Pressure-Volume (PV) Loop and System Hemodynamics}

Whereas the four cardiac cycle phases discussed earlier are clearly illustrated in the Wiggers diagram, which illustrates the time series behavior of the left ventricular pressure (LVP), left atrial pressure (LAP), aortic pressure (AoP), and left ventricular volume (LVV) during the cardiac cycle, there is another advantageous relationship called pressure-volume (PV) loop that relates between LVP and LVV. This relationship is best explained by plotting the simultaneous measurements of LVP and LVV, where LVV represents the $\mathrm{x}$ - 
axis and LVP represents the y-axis in this relationship. Figure 2.6 shows a typical PV loop (lower panel) that corresponds to a given time representation for LVP and LVV (upper panel). The four cardiac cycle phases are clearly demonstrated in the PV loop shown in this figure. The phases labeled b, c, d, and a on the PV loop in Figure 2.6 respectively represent the isovolumic contraction phase, the ejection phase, the isovolumic relaxation phase, and the filling phase.
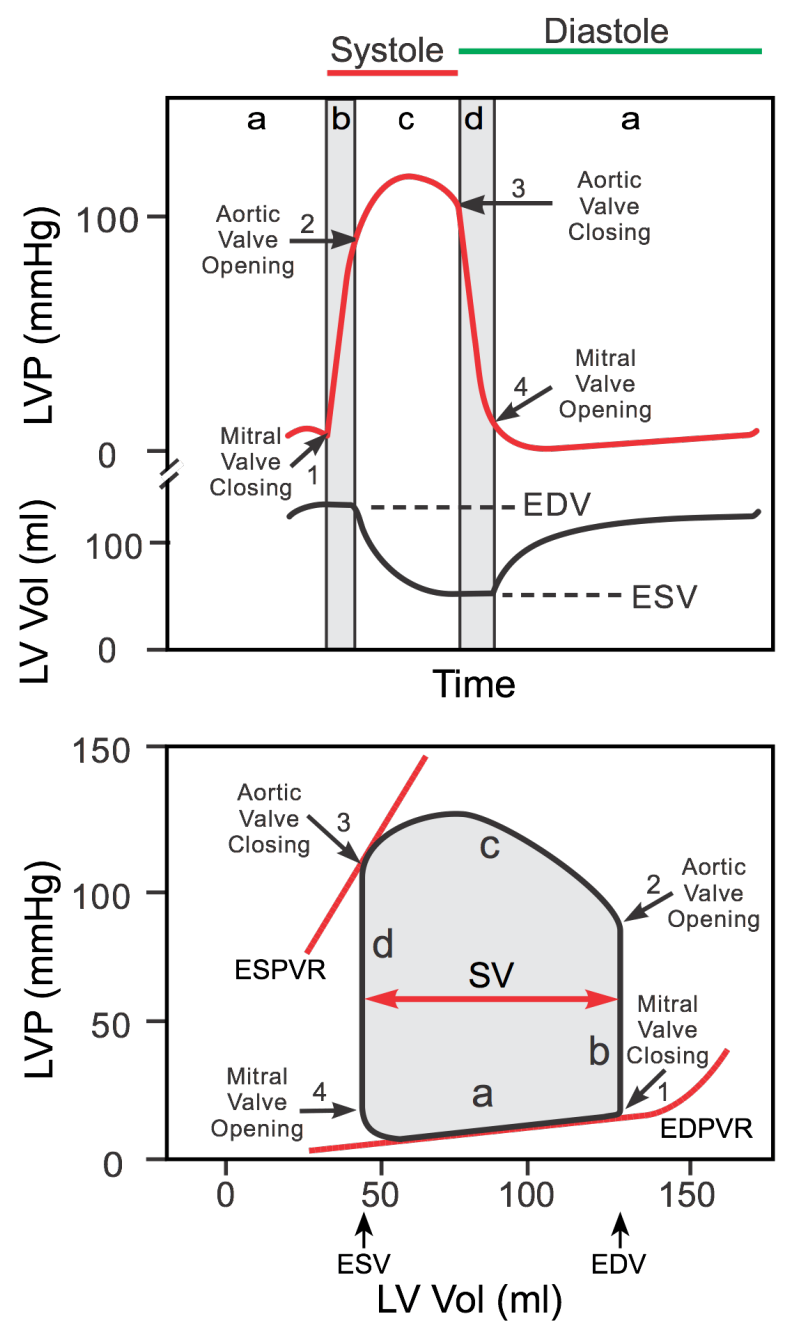

Figure 2.6. Time series representation of left ventricular pressure and volume (upper panel) and the corresponding PV-loop (lower panel). The labels "b", "c", "d", and "a" represent the isovolumic contraction phase, ejection phase, isovolumic relaxation phase, and filling phase, respectively [55]. 
There are several physiological measurements that can be retrieved from a given PV loop. The maximum value on the x-axis (LVV axis) in Figure 2.7 is called the enddiastolic volume (EDV), which represents the maximum volume of the left ventricle during the cardiac cycle, specifically at the end of the filling phase. On the other hand, the endsystolic volume (ESV) is the minimum volume of the left ventricle during the cardiac cycle which occurs at the end of the ejection phase. EDV and ESV are clearly illustrated on the $\mathrm{x}$-axis in Figure 2.7. The difference between these two values is called the stroke volume $(\mathrm{SV})$ as follows:

$$
S V=E D V-E S V
$$

where SV is a measurement representing the amount of blood ejected during one cardiac cycle. The cardiac output (CO), which represents the amount of blood circulated through the body in one minute, can then be calculated by multiplying SV by the heart rate (HR):

$$
C O=S V * H R
$$

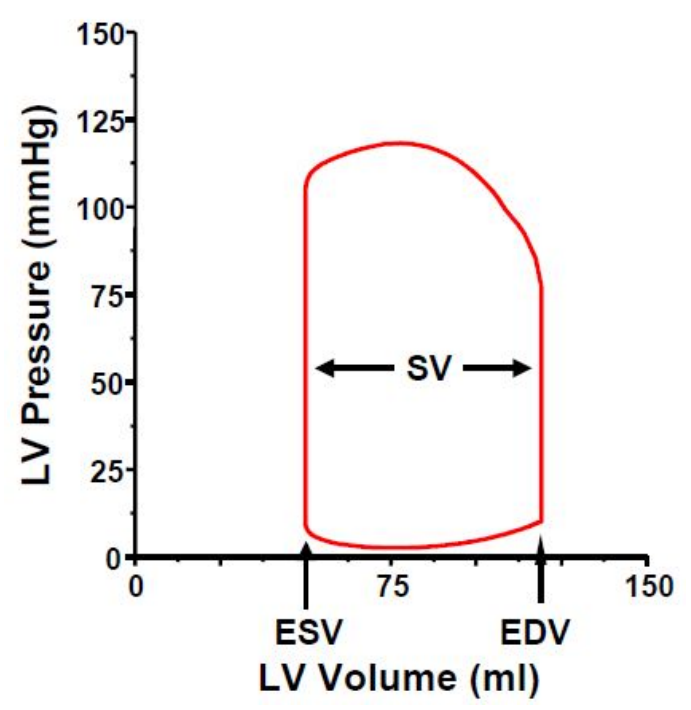

Figure 2.7. Determining the stroke volume (SV) from the PV-loop [55]. 
Another important physiological measurement that can be retrieved from the PV loop is the diastolic (DBP) and systolic (SBP) blood pressures. Considering the y-axis (LVP axis) of the PV loop shown in Figure 2.8, the DBP and SBP shown respectively represent the aortic pressure at the onset of blood ejection and the maximum aortic pressure, assuming that left ventricular pressure and aortic pressure are equal during ejection. Moreover, Pes and EDP, also shown in Figure 2.8, are also of great importance. Pes, end-systolic pressure, represents the pressure in the LV at the end of the ejection phase, right at the aortic valve closure, whereas EDP, end-diastolic pressure, represents the pressure in the LV at the end of filling phase, the moment when the mitral valve closes. Pes represents the point of time of maximum stiffness whereas EDP represents the minimum stiffness. Thus, stiffness (also known as elastance) varies during one cardiac cycle. Elastance, the reciprocal of compliance, is defined as the change in pressure that results from a given change in volume within a chamber. Its maximal and minimal values occur at the end of the systolic and diastolic phases, respectively [55].

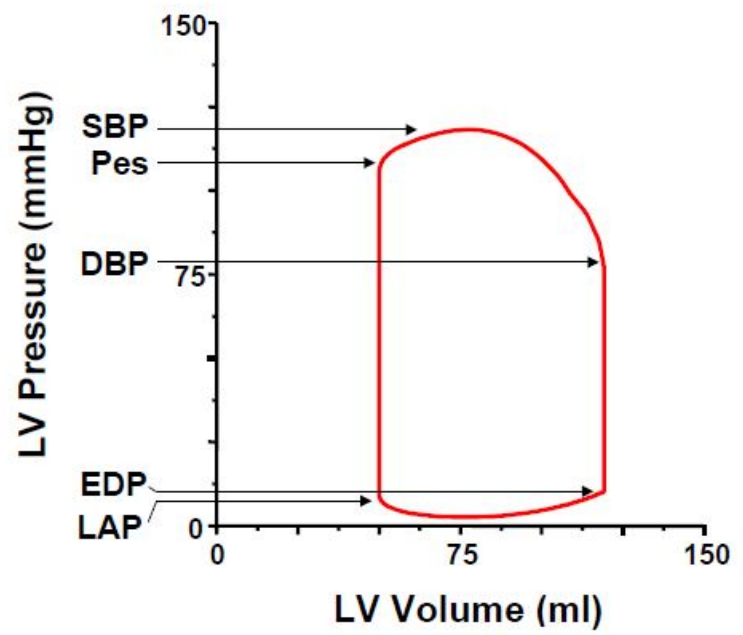

Figure 2.8. Reading systolic blood pressures (SBP), diastolic blood pressures (DBP), end-systolic pressure (Pes ), and end-diastolic pressures (EDP) from the PV-loop [55]. 
EDP and EDV introduce a new relationship called end-diastolic pressure-volume relationship (EDPVR). On the other hand, Pes and ESV introduce another relationship called end-systolic pressure-volume relationship (ESPVR). The literature indicates that EDPVR is a nonlinear relationship, whereas ESPVR is a linear one [55]. While EDPVR bounds the lower right corner of the PV loop, ESPVR bounds the upper left corner of the PV loop as shown in Figure 2.9. The slope of ESPVR represents the maximum value of elastance, whereas the slope of EDPVR represents the minimum value of elastance.

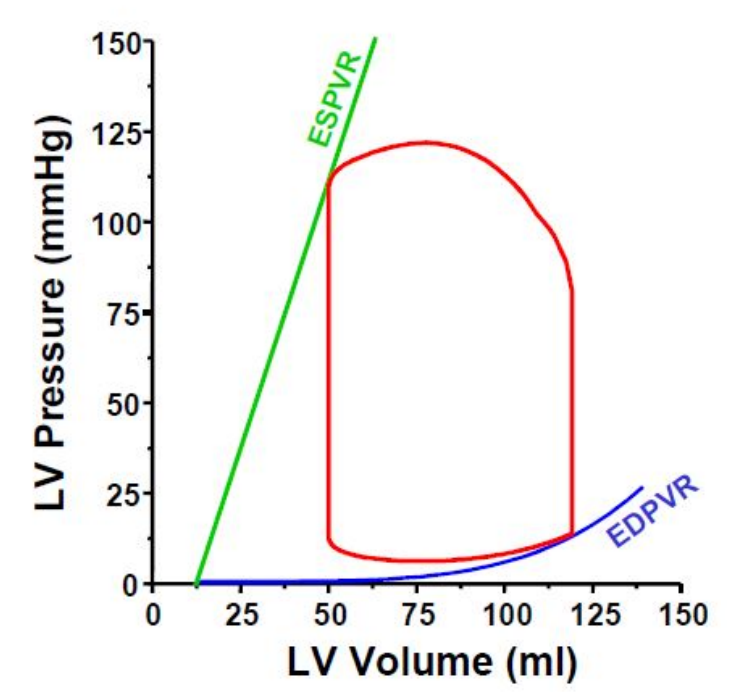

Figure 2.9. Finding the end-systolic pressure-volume relationship (ESPVR) and end-diastolic pressurevolume relationship (EDPVR) from the PV-loop [55].

The time series representation of the instantaneous relationship between the left ventricular pressure and left ventricular volume is called the time-varying elastance $E(t)$. A rough approximation for a typical time-varying elastance function during one cardiac cycle is shown in Figure 2.10. $\mathrm{E}_{\max }$ and $\mathrm{E}_{\min }$ represent the slopes of ESPVR and EDPVR, respectively. Knowledge of the time-varying elastance $E(t)$ is of great importance. It can 
allow us to predict the pressure at any time during the cardiac cycle if the volume is known or vice versa.

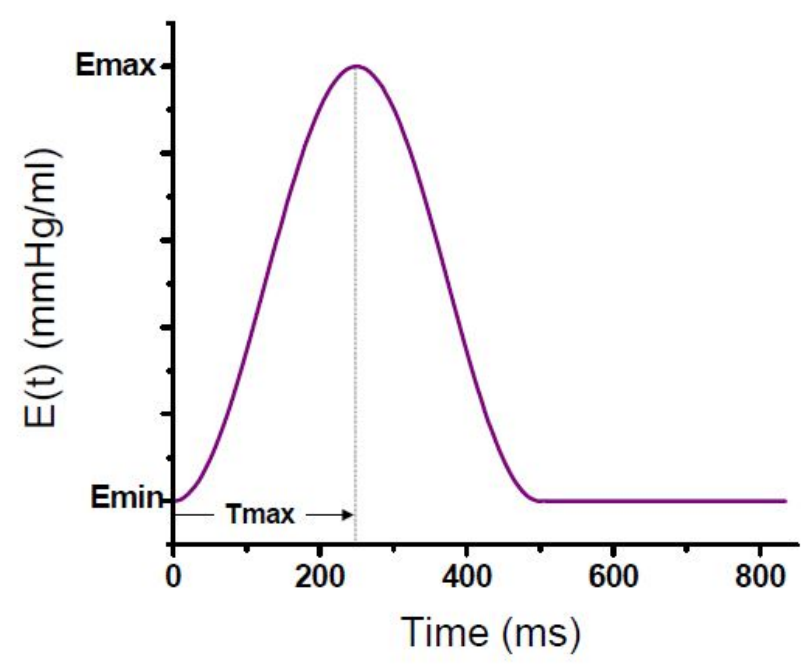

Figure 2.10. Computing elastance function from the PV-loop [55]. 


\section{CHAPTER 3 Modeling the Cardiovascular System}

A simplified cardiovascular system, consisting of the left heart (atrium and ventricle) and the body's arterial system, is modeled as a lumped-parameter electric circuit [56]. In this modeling work, preload is modeled as a single, time-varying compliance of the left ventricle as well as a constant compliance representing the left atrium. Afterload, representing the arterial system, is represented by a four-element Windkessel model.

In this chapter, the lumped-parameter modeling process for the cardiovascular system is described. The model is then validated by reproducing hemodynamics that are consistent with clinical data. The chapter is concluded with preliminary studies demonstrating two opportunities for $\mathrm{RH}$ treatment that involve implementing the cardiac contractility modulation technology and the addition of artificial compliance. These preliminary studies of treating $\mathrm{RH}$ are for demonstration purposes and do not represent the focus of this research.

\subsection{Analogies between the Circulatory System and Electric Circuits}

Researchers in physiology use electric circuits to describe their models of the blood circulatory system [52]. We are accustomed to viewing the blood circulatory system as a hydraulic system, where the heart represents a pump, and the vessels represent the pipes for the flow of blood. Fluid and electricity represent a clear demonstration in conceptualizing some processes, such as the blood circulatory system. For this reason, it is important to understand the analogies between hydraulic and electrical systems. 
A basic description for the action of the aorta in terms of electric circuit representation is illustrated in Figure 3.1. The left ventricle, which represents an intermittent pump for the system, is modeled as a variable voltage source. The aorta is modeled as a Windkessel model that consists of resistors and capacitors and in between the left ventricle and aorta, there is the aortic valve which is modeled as a diode to mimic the unidirectional flow of blood and prevent the back flow to the left ventricle.
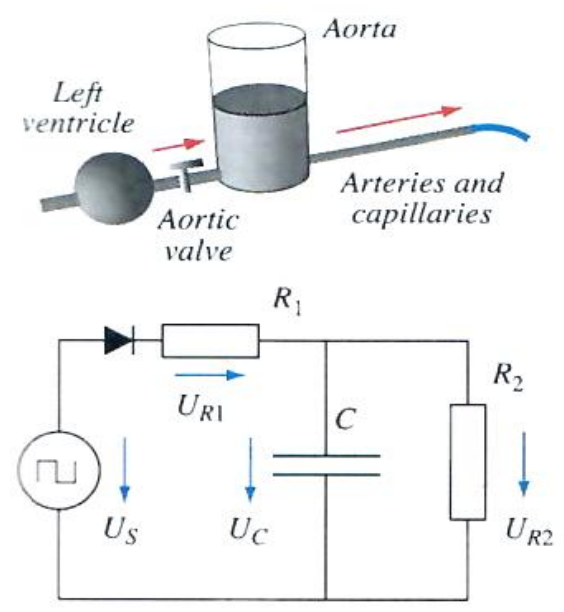

Figure 3.1. A Windkessel representation of the circulatory system (top) and its equivalent electric circuit representation (bottom) [52].

The analogies between the circulatory system and electric circuits and their respective modeling methodology will be described next with more details.

\subsubsection{Modeling a Vessel}

The basic idea of modeling the hemodynamics of a vessel is shown in part (A) of Figure 3.2. The relationship between blood pressure and blood flow rate can be perfectly described by the relationship between voltage and current in electric circuit theory, whereas blood volume corresponds to electric charge. The other characteristics of the 
cardiovascular system's hemodynamics can be represented by an RLC electric circuit as shown in part (B) of Figure 3.2. In this analogy, the arterial compliance, vascular resistance, and blood inertia (which represents the mass of the blood undergoing acceleration) correspond to capacitance, resistance, and inductance, respectively.

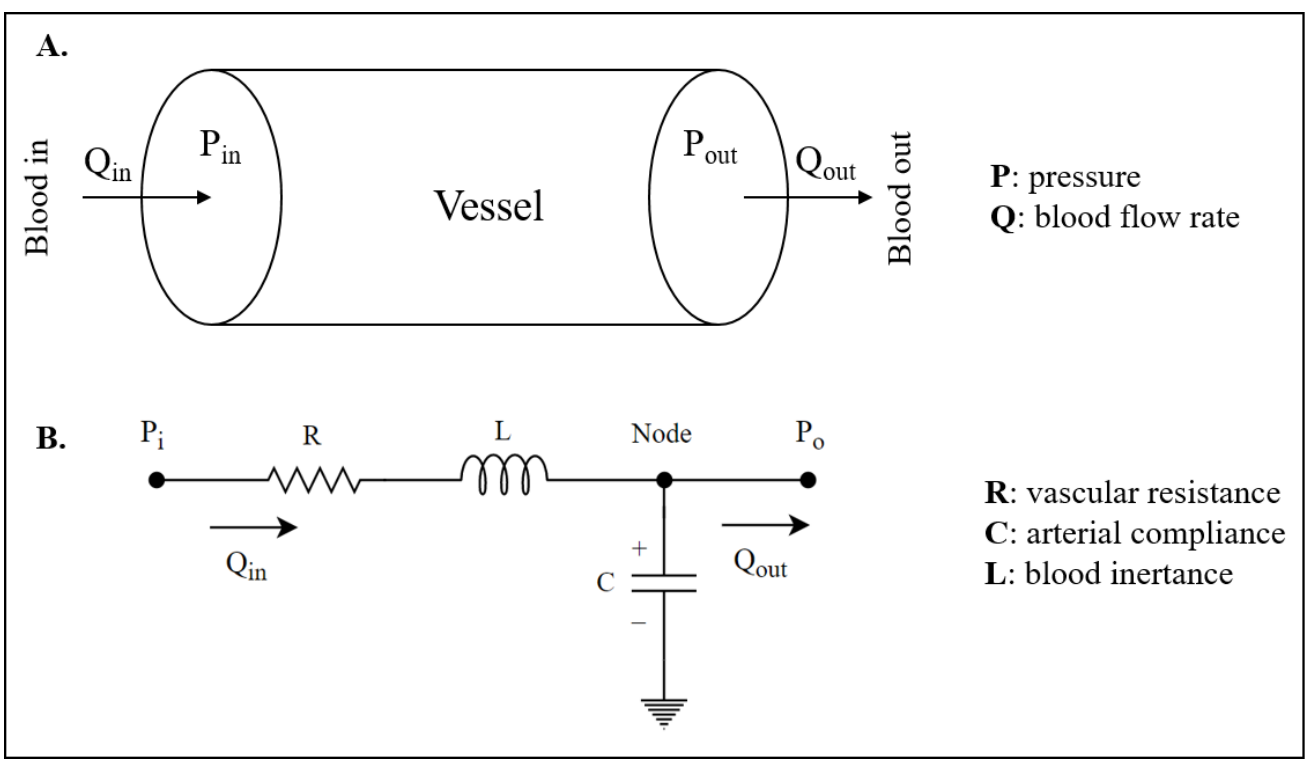

Figure 3.2. Electrical analogue of vascular elements. Hemodynamic elements of a blood vessel are shown in (A), and the corresponding equivalent electric circuit elements are shown in (B). Figure was reproduced from [57].

This lumped-parameter model of representing the hemodynamic elements of the cardiovascular system by their equivalent electric circuit elements allows for the application of Ohm's law and Kirchhoff's laws of node currents and loop voltages [57]. This greatly facilitates the derivation of key dynamic equations describing the hemodynamics of the cardiovascular system for the sake of implementation in computer simulations. This can lead to the equations presented below:

$$
Q=\frac{P_{i}-P_{0}}{R}
$$




$$
\begin{gathered}
\frac{d V}{d t}=Q_{\text {in }}-Q_{\text {out }} \\
P=\frac{V}{C}
\end{gathered}
$$

where $Q$ is the blood flow rate, $V$ is the volume, $P$ is the pressure, $R$ is the body's systemic resistance, and $C$ is the arterial compliance.

\subsubsection{Modeling Heart Valves}

Left heart valves, mitral and aortic, can be represented by diodes as shown in Figure 3.3. A diode is an electric switch that allows current to flow in only one direction and can conduct only when the voltage in its anodic (positive) terminal is higher than that in the cathodic (negative) terminal. The blood flow through the mitral valve, $Q_{m}$, and through the aortic valve, $Q_{a}$, can be represented according to equations (3.4) and (3.5) where $R_{m}$ and $R_{a}$ respectively represent the resistance of the mitral valve and aortic valve against blood flow.

$$
\begin{aligned}
& Q_{m}= \begin{cases}\frac{L A P-L V P}{R_{m}}, & \text { when } L A P>L V P(\text { filling }) \\
0, & \text { else }\end{cases} \\
& Q_{a}= \begin{cases}\frac{L V P-A o P}{R_{a}}, & \text { when } L V P>A o P(\text { ejection }) \\
0, & \text { else }\end{cases}
\end{aligned}
$$




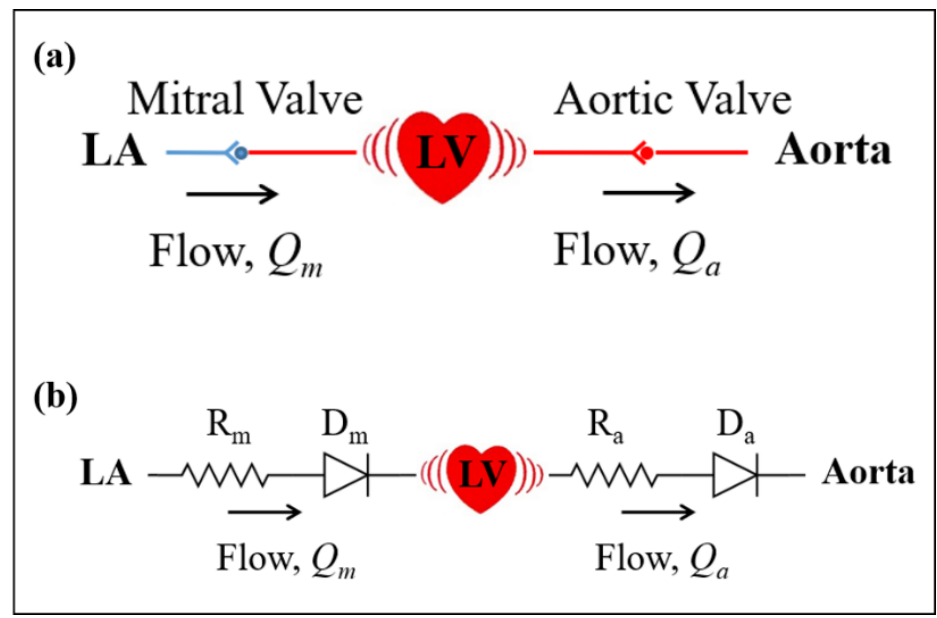

Figure 3.3. Modeling the mitral and aortic valves. The valves are represented by check valves according to hydraulic systems theory (a) and diodes in series with resistors according to electric circuits theory (b).

\subsubsection{Modeling the Heart}

In the cardiovascular system, the ventricle represents a chamber in the heart that has an entrance valve to allow for blood to fill and another exit valve to allow for pumping blood out to circulatory systems. Considering only the left ventricle in this modeling procedure, which is considered the primary driving force of this system, the two valves that are associated with the LV are called the mitral valve and aortic valve. The mitral valve allows blood to flow from the LA to fill the LV, whereas the aortic valve allows the LV to pump blood out across it into the arterial system. These valves are represented by two diodes (Figure 3.3), with one representing the mitral valve and located before (to the left of) the LV while the other represents the aortic valve which is located after (to the right of) the LV. The diode is simply a switch that can either be closed (conducting) or open (isolating), thus a closed valve is electrically equivalent to an open-circuit (isolating) switch, whereas an open valve is equivalent to a closed-circuit (conducting) switch. Each diode is associated with a resistor representing the actual resistance that opposes the blood 
flow through the valve. Thus, we will have different combinations for the states of these two diodes depending on the cardiac cycle phase as described in Table 3.1.

\begin{tabular}{l|c|c|c|c}
\hline \multirow{2}{*}{ Cardiac cycle phases } & \multicolumn{2}{c|}{ Valves state } & Duration \\
\cline { 3 - 4 } & & Mitral $\left(\mathrm{D}_{\mathrm{m}}\right)$ & Aortic $\left(\mathrm{D}_{\mathrm{a}}\right)$ & $(\mathbf{s e c})$ \\
\hline Isovolumic contraction & \multirow{3}{*}{ Systole } & Closed & Closed & $\sim 0.05$ \\
\cline { 3 - 4 } Ejection & & Closed & Open & $\sim 0.26$ \\
\hline \multirow{2}{*}{ Isovolumic relaxation } & \multirow{2}{*}{ Diastole } & Closed & Closed & $\sim 0.08$ \\
\cline { 3 - 4 } Filling & & Open & Closed & $\sim 0.41$ \\
\hline \multicolumn{3}{l}{ Cardiac cycle interval $(\mathrm{sec})$} & 0.8 \\
\hline
\end{tabular}

Table 3.1. Phases of the cardiac cycle and valve states during each phase, with approximate durations for each phase based on heart rate of 75 beats/min [58].

The pumping action of the LV varies during the cardiac cycle and can be represented by a time-varying compliance or capacitance, $C(t)$. This can be achieved by considering the time-varying elastance function $E(t)$, the reciprocal of compliance $C(t)$, which is a measure of the change in pressure resulting from a given change in volume within a chamber. The left ventricular elastance is bounded by a maximal value that occurs during end-systole and a minimal value that occurs during end-diastole. The elastance function used in this model follows the model of Suga and Sagawa [59] according to the following equation:

$$
E(t)=\frac{L V P(t)}{L V V(t)-V_{0}}
$$

where $L V P$ and $L V V$ are the left ventricular pressure and volume, respectively. $V_{0}$ is a theoretical value representing the volume of the LV when the pressure is zero. The 
mathematical model used for the elastance function followed the one used in [56] according to the following:

$$
E(t)=\left(E_{\max }-E_{\min }\right) * E_{n}\left(t_{n}\right)+E_{\text {min }}
$$

where $E_{\max }$ and $E_{\min }$ are the maximum and minimum values of the elastance function and represent the end-systolic pressure-volume relationship (ESPVR) and end-diastolic pressure-volume relationship (EDPVR), respectively. $E_{n}\left(t_{n}\right)$ is the normalized timevarying elastance function which is approximated by the periodic "double-Hill" function [60] according to the following equation:

$$
E_{n}\left(t_{n}\right)=1.55\left[\frac{\left(\frac{t_{n}}{0.7}\right)^{1.9}}{1+\left(\frac{t_{n}}{0.7}\right)^{1.9}}\right]\left[\frac{1}{1+\left(\frac{t_{n}}{1.17}\right)^{21.9}}\right]
$$

where $t_{n}=\frac{t}{T_{\max }}, T_{\max }=0.2+0.15 * t_{c}$, with $t_{c}$ defined as the cardiac cycle period $\left(t_{c}=\right.$ $\left.\frac{60}{H R}\right) ; H R$ is the heart rate. The corresponding plot of the elastance function, where the values used for $E_{\max }, E_{\min }$, and $H R$ are $2.5 \mathrm{mmHg} / \mathrm{ml}, 0.06 \mathrm{mmHg} / \mathrm{ml}$, and 75 beats $/ \mathrm{min}$ respectively, is shown in Figure 3.4. 


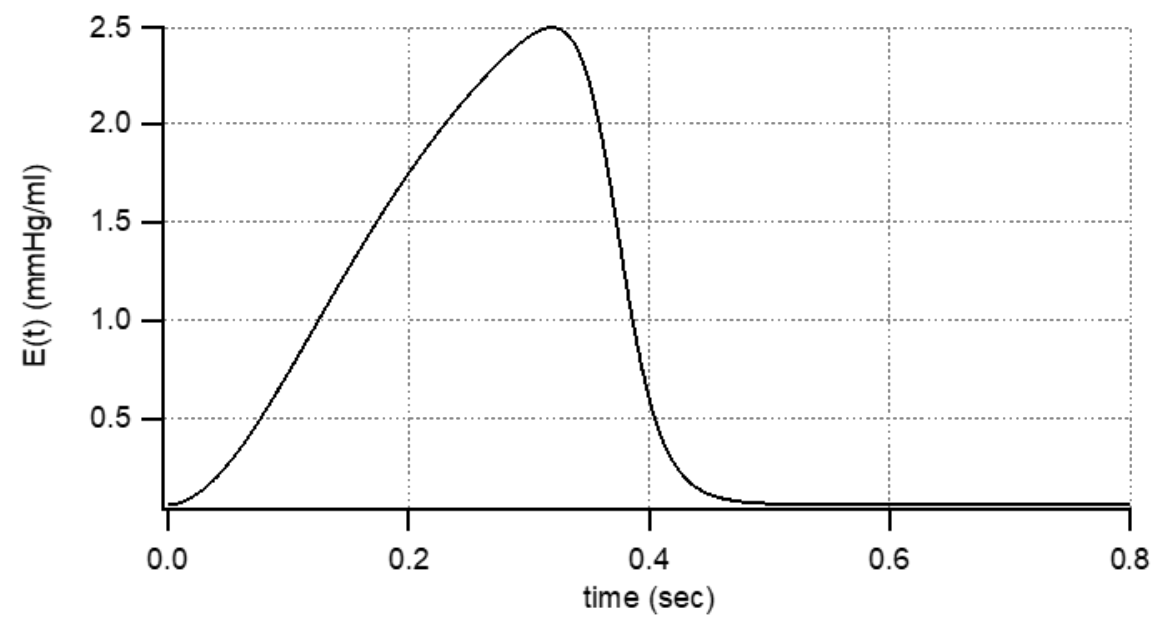

Figure 3.4. Elastance function $\mathrm{E}(\mathrm{t})$ which describes the time-varying relationship between blood pressure and volume in the left ventricle during one cardiac cycle.

The left atrium in this modeling procedure is represented by a constant compliance (capacitance).

\subsubsection{Modeling the Arterial Network}

The arterial system is modeled by a lumped-parameter circuit 4-element Windkessel model as shown in Figure 3.5. The four elements forming the 4-element WK model are the characteristic impedance of the aorta "Rc", the inertance of blood "L", the arterial compliance "C", and the peripheral resistance "Rp".

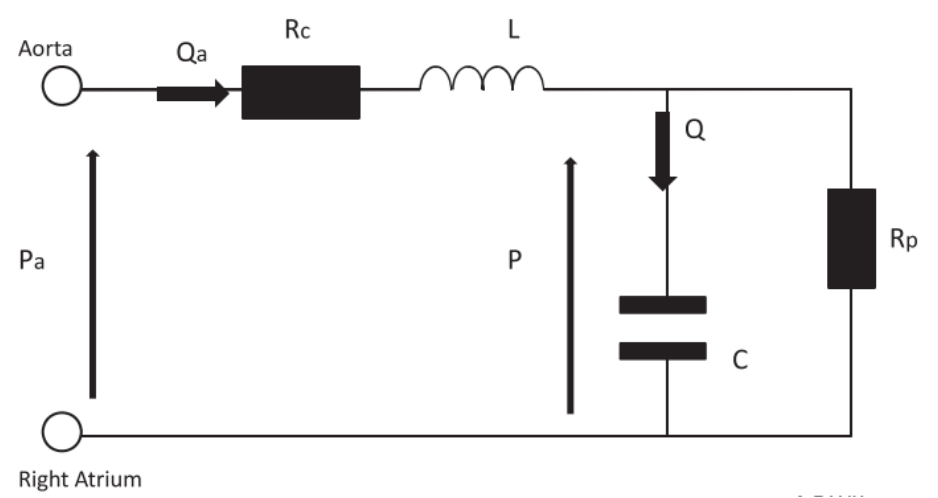

Figure 3.5 Four-element Windkessel model representing the arterial network in the cardiovascular system [41]. 


\subsection{Lumped-Parameter Model of the Cardiovascular System}

The full model that describes the cardiovascular system explained earlier is shown in Figure 3.6. In this model, $C_{1}(t)$ represents the time-varying compliance of the LV, which is the reciprocal of the elastance function described in Figure 3.4, while $C_{2}$ and $C_{3}$ are constant compliance values representing the left atrial compliance and arterial compliance, respectively. $C_{4}$ represents the compliance of the proximal part of the aorta as shown in the model used in [51]. $D_{m}$ and $D_{a}$ are diodes representing the mitral valve and aortic valve respectively, while $R_{m}$ and $R_{a}$ represent the resistances that oppose the blood flowing through the mitral valve and aortic valve, respectively. $L$ is an inductance that represents the inertia of blood. $R_{c}$ and $R_{p}$ are the characteristic impedance of the aorta and the body's peripheral resistance, respectively.

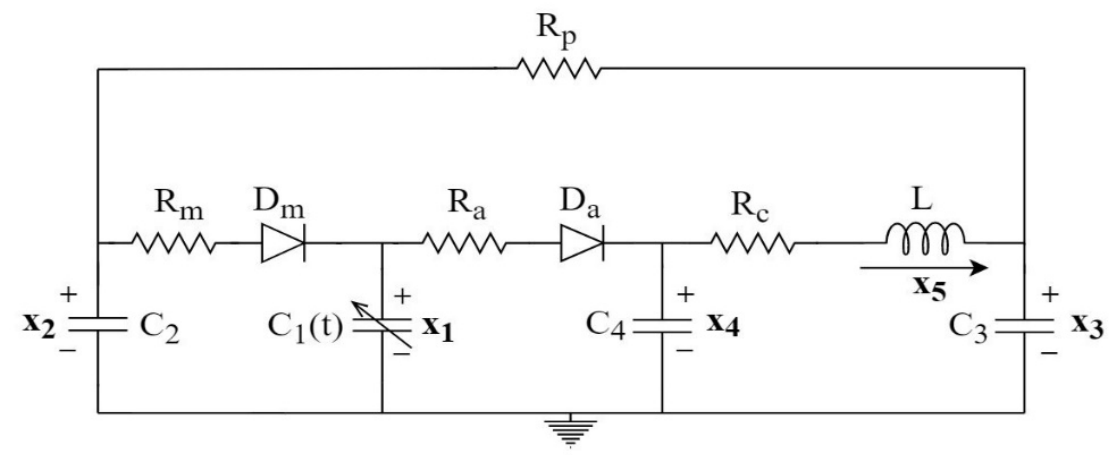

Figure 3.6 Lumped-parameter electric circuit model of the cardiovascular system.

\subsection{State-Space Representation}

State-space representation is used here to analyze the lumped-parameter circuit model of the cardiovascular system (Figure 3.6). The five state variables in this system model are the left ventricular pressure (LVP), left atrial pressure (LAP), arterial pressure 
(AP), aortic pressure $(\mathrm{AoP})$, and aortic flow $\left(\mathrm{Q}_{\mathrm{A}}\right)$, as shown in Table 3.2. Thus, the states vector $X$ is:

$$
X=\left[\begin{array}{l}
x_{1} \\
x_{2} \\
x_{3} \\
x_{4} \\
x_{5}
\end{array}\right]=\left[\begin{array}{c}
L V P \\
L A P \\
A P \\
A o P \\
Q_{A}
\end{array}\right]
$$

\begin{tabular}{c|l|c}
\hline $\begin{array}{c}\text { State-space } \\
\text { variable }\end{array}$ & \multicolumn{1}{|c}{ Physiological meaning (unit) } & Abbreviation \\
\hline$x_{1}$ & Left ventricular pressure $(\mathrm{mm} \mathrm{Hg})$ & LVP \\
\hline$x_{2}$ & Left atrial pressure $(\mathrm{mm} \mathrm{Hg})$ & LAP \\
\hline$x_{3}$ & Arterial pressure $(\mathrm{mm} \mathrm{Hg})$ & AP \\
\hline$x_{4}$ & Aortic pressure $(\mathrm{mm} \mathrm{Hg})$ & AoP \\
\hline$x_{5}$ & Aortic flow $(\mathrm{ml} / \mathrm{sec})$ & $\mathrm{Q}_{\mathrm{A}}$ \\
\hline
\end{tabular}

Table 3.2. State variables used in the cardiovascular system model.

The state equations can now be derived for each of the phases described in Table 3.1. Depending on the state of the mitral and aortic valves, an equivalent circuit describing each phase will be shown next. Kirchhoff's laws of node currents and loop voltages, as well as Ohm's law, are then applied to solve for the state equations for each circuit.

\section{Isovolumic contraction phase}

This phase starts the moment when the mitral valve is closed after filling the LV with blood, and so both the mitral and aortic valves are closed during this phase. The left ventricular contraction will intuitively increase the pressure inside the left ventricular chamber, while its blood volume remains unchanged since both valves are closed. This will cause a rapid increase in the left ventricular pressure (LVP) during this phase from around 
$10 \mathrm{mmHg}$, which is the moment when the mitral valve closes, to around $80 \mathrm{mmHg}$, when the aortic valve opens. The equivalent circuit model representing this phase is shown in Figure 3.7, and a more simplified circuit model is shown in Figure 3.8.

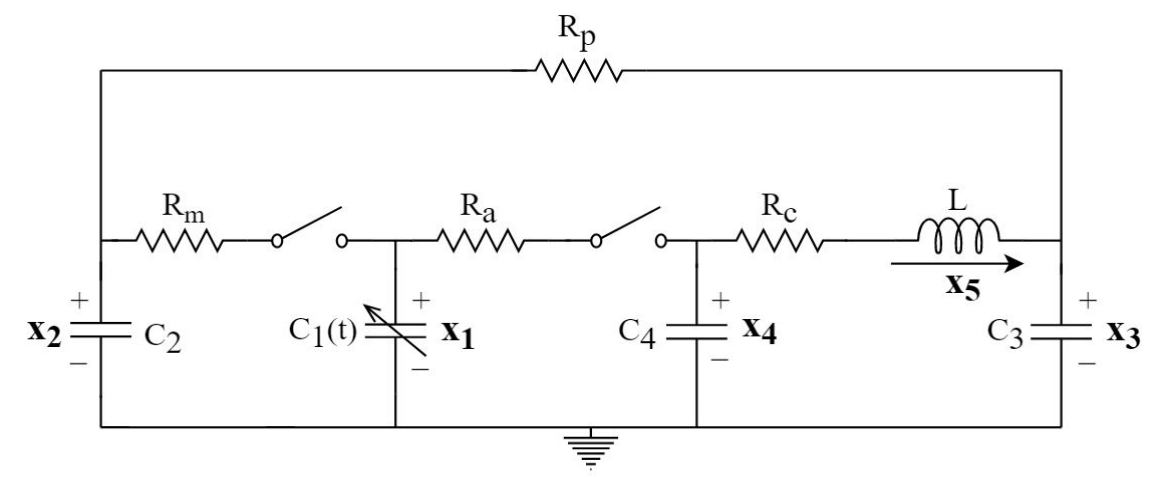

Figure 3.7. Equivalent circuit for the isovolumic contraction phase. Both the mitral and aortic valves are closed, thus both diodes are open-circuited.

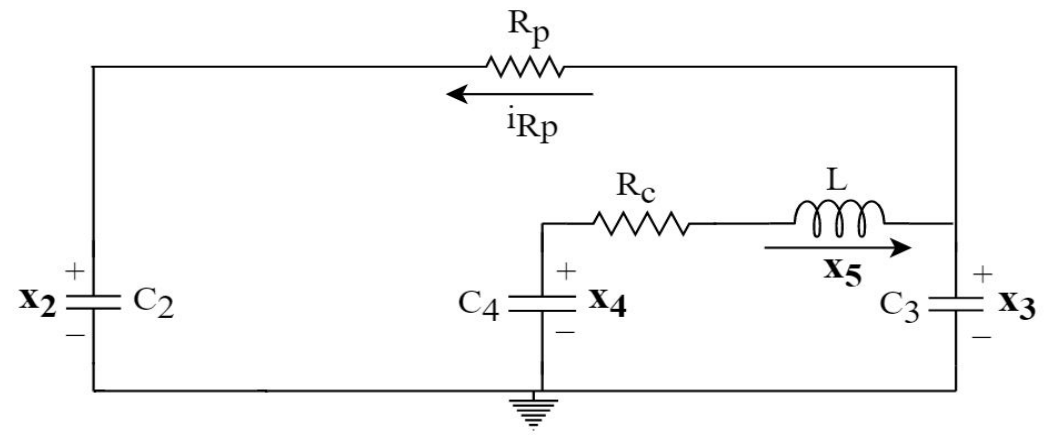

Figure 3.8. Simplified electric circuit model for the isovolumic phase.

It is known that the current " $i$ " flowing through a capacitor " $C$ " is described as:

$$
i=C \frac{d v}{d t}
$$

where " $v$ " is the voltage across the capacitor, and so the current flowing through the resistor $R_{p}$ in the direction shown in Figure 3.8 is:

$$
i_{R p}=C_{2} \frac{d x_{2}}{d t}=x_{5}-C_{3} \frac{d x_{3}}{d t}
$$


To solve for $\frac{d x_{1}}{d t}$, or simply $\dot{x}_{1}$, where $x_{1}$ represents the left ventricular pressure, we use equation (3.6) of the elastance function which can be re-written as:

$$
L V P(t)=x_{1}(t)=E(t) *\left(L V V(t)-V_{0}\right)
$$

Taking the derivative of both sides of equation (3.11) with respect to time, we get:

$$
L \dot{V} P(t)=\dot{x}_{1}(t)=\dot{E}(t) *\left(L V V(t)-V_{0}\right)+E(t) * L \dot{V} V(t)
$$

We can easily infer that $L \dot{V} V(t)=0$ during the isovolumic contraction phase since blood volume inside the LV does not change as both the mitral valve and aortic valve are closed, thus the above equation can be re-written as follows:

$$
L \dot{V} P(t)=\dot{E}(t) *\left(L V V(t)-V_{0}\right)=\frac{\dot{E}(t)}{\frac{L V P(t)}{\left(L V V(t)-V_{0}\right)}} * L V P(t)=\frac{\dot{E}(t)}{E(t)} * L V P(t)
$$

Which can also be re-written according to the following:

$$
\dot{x}_{1}(t)=\frac{\dot{E}(t)}{E(t)} * x_{1}(t)
$$

To solve for $\dot{x}_{2}$, the rate of change in the state representing the left atrial pressure, we use $i_{R_{p}}=C_{2} \frac{d x_{2}}{d t}=C_{2} * \dot{x}_{2}$ from equation (3.10). Applying Kirchhoff's voltage law (KVL) on the outer loop of the circuit shown in Figure 3.8, we obtain $x_{2}-x_{3}+R_{p} *\left(C_{2} * \dot{x}_{2}\right)=0$, which leads to:

$$
\dot{x}_{2}(t)=\frac{-1}{R_{p} C_{2}} * x_{2}(t)+\frac{1}{R_{p} C_{2}} * x_{3}(t)
$$


Applying Kirchhoff's voltage law (KVL) again to solve for $\dot{x}_{3}$ (where $x_{3}$ represents the arterial pressure), using $i_{R_{p}}=x_{5}-C_{3} \frac{d x_{3}}{d t}=-C_{3} * \dot{x}_{3}$, also from equation (3.10), we get $x_{2}-x_{3}+R_{p} *\left(x_{5}-C_{3} * \dot{x}_{3}\right)=0$, which leads to:

$$
\dot{x}_{3}(t)=\frac{1}{R_{p} C_{3}} * x_{2}(t)-\frac{1}{R_{p} C_{3}} * x_{3}(t)+\frac{1}{C_{3}} * x_{5}(t)
$$

We can easily derive the state equation for $\dot{x}_{4}$ from Figure 3.8 by applying equation (3.9) according to the following:

$$
\dot{x}_{4}(t)=\frac{-1}{C_{4}} * x_{5}(t)
$$

Finally, to solve for the state equation for the fifth state variable (aortic flow), we apply Kirchhoff's voltage law (KVL) on the inner loop of the circuit shown in Figure 3.8, where we get $-x_{4}+R_{c} * x_{5}+L * \dot{x}_{5}+x_{3}=0$. This can be re-written as shown below:

$$
\dot{x}_{5}(t)=\frac{-1}{L} * x_{3}(t)+\frac{1}{L} * x_{4}(t)-\frac{R_{C}}{L} * x_{5}(t)
$$

Putting equations (3.13), (3.14), (3.15), (3.16), and (3.17) together to solve for the matrix $A(t)$, where $\dot{x}(t)=A(t) * x(t)$, we obtain:

$$
\left[\begin{array}{l}
\dot{x}_{1}(t) \\
\dot{x}_{2}(t) \\
\dot{x}_{3}(t) \\
\dot{x}_{4}(t) \\
\dot{x}_{5}(t)
\end{array}\right]=\left[\begin{array}{ccccc}
\frac{\dot{E}(t)}{E(t)} & 0 & 0 & 0 & 0 \\
0 & \frac{-1}{R_{p} C_{2}} & \frac{1}{R_{p} C_{2}} & 0 & 0 \\
0 & \frac{1}{R_{p} C_{3}} & \frac{-1}{R_{p} C_{3}} & 0 & \frac{1}{C_{3}} \\
0 & 0 & 0 & 0 & \frac{-1}{C_{4}} \\
0 & 0 & \frac{-1}{L} & \frac{1}{L} & \frac{-R_{c}}{L}
\end{array}\right] *\left[\begin{array}{l}
x_{1}(t) \\
x_{2}(t) \\
x_{3}(t) \\
x_{4}(t) \\
x_{5}(t)
\end{array}\right]
$$


Thus $A_{1}(t)$, where the subscript " 1 " stands for the isovolumic contraction phase, can be written as:

$$
A_{1}(t)=\left[\begin{array}{ccccc}
\frac{\dot{E}(t)}{E(t)} & 0 & 0 & 0 & 0 \\
0 & \frac{-1}{R_{p} C_{2}} & \frac{1}{R_{p} C_{2}} & 0 & 0 \\
0 & \frac{1}{R_{p} C_{3}} & \frac{-1}{R_{p} C_{3}} & 0 & \frac{1}{C_{3}} \\
0 & 0 & 0 & 0 & \frac{-1}{C_{4}} \\
0 & 0 & \frac{-1}{L} & \frac{1}{L} & \frac{-R_{c}}{L}
\end{array}\right]
$$

where $E(t)$, the reciprocal of the compliance $C_{1}(t)$ in the cardiovascular model, is the elastance function (Figure 3.4) that describes the behavior of the left ventricular function. $\dot{E}(t)$ is the derivative of the elastance function. $C_{2}, C_{3}$, and $C_{4}$ are the left atrial compliance, arterial compliance, and aortic compliance, respectively. $R_{p}$ and $R_{c}$ are the peripheral resistance and characteristic resistance respectively, whereas $L$ is the inductance that represents blood inertia.

\section{Ejection phase}

During the ejection phase, the mitral valve remains closed while the aortic valve opens the moment the pressure in the LV becomes greater than that in the aorta, and so the left ventricle will start pumping the blood out to the body's circulatory system through the aortic valve. The equivalent circuit for the "ejection" phase, which shows that the mitral valve " $\mathrm{D}_{\mathrm{m}}$ " is open-circuited and the aortic valve " $\mathrm{D}_{\mathrm{a}}$ " is closed-circuited as indicated in Table 3.1, is shown in Figure 3.9 while its simplified version is shown in Figure 3.10. 


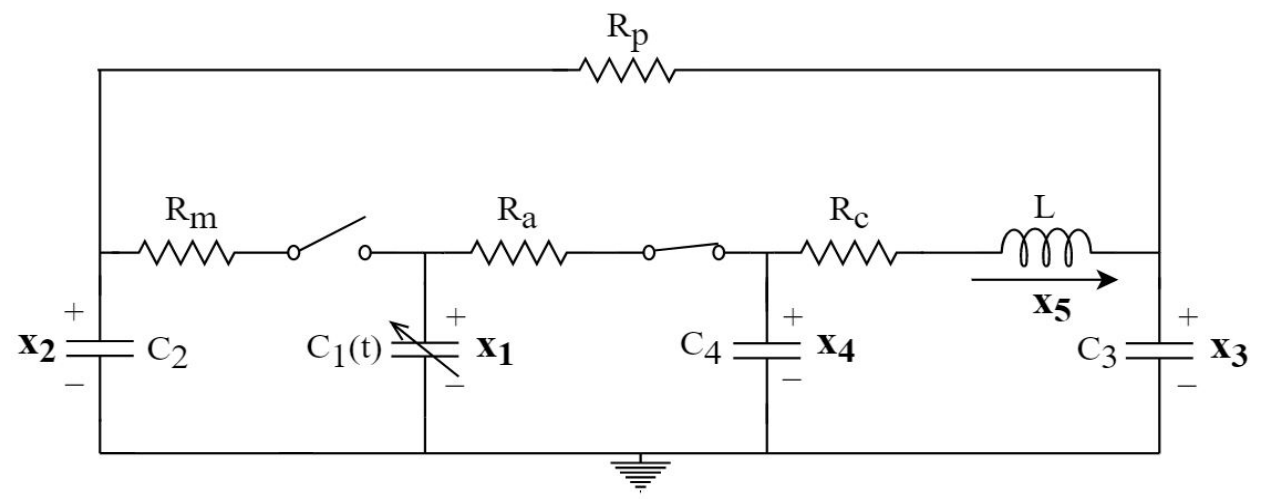

Figure 3.9. Equivalent circuit for the ejection phase. The mitral valve is still closed (open-circuited) while the aortic valve is open (short-circuited).

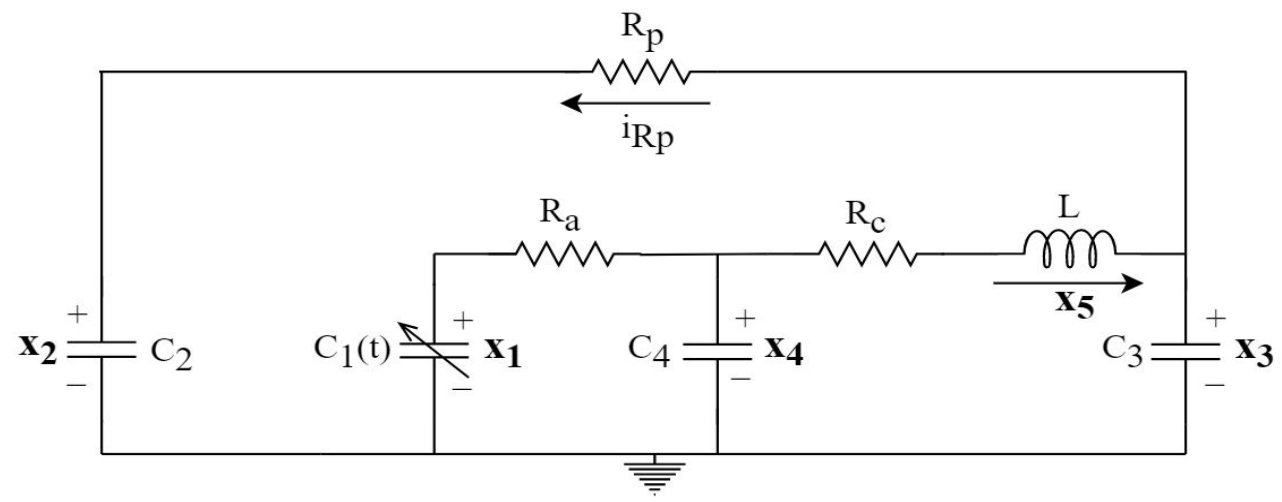

Figure 3.10. Simplified circuit for the ejection phase.

The current $i_{R_{p}}$, flowing in the direction shown in Figure 3.10, is:

$$
i_{R_{p}}=C_{2} \frac{d x_{2}}{d t}=x_{5}-C_{3} \frac{d x_{3}}{d t}
$$

where $x_{5}$ represents the aortic flow. Using equation (3.6) of the elastance function $E(t)$ to solve for $\dot{x}_{1}$, we showed earlier that differentiating both sides with respect to time yielded equation (3.12) which is shown below:

$$
L \dot{V} P(t)=\dot{x}_{1}(t)=\dot{E}(t) *\left(L V V(t)-V_{0}\right)+E(t) * L \dot{V} V(t)
$$


$L \dot{V} V(t)$, the rate of change of the LV volume, is not zero during this phase as in the case of the isovolumic contraction phase but rather can be described according to the following equation:

$$
\begin{gathered}
L \dot{V} V(t)=Q_{\text {in }}-Q_{\text {out }}=0-\left(\frac{x_{1}(t)-x_{4}(t)}{R_{a}}\right) \\
L \dot{V} V(t)=-\frac{1}{R_{a}} * x_{1}(t)+\frac{1}{R_{a}} * x_{4}(t)
\end{gathered}
$$

which yields:

$$
\begin{gathered}
L \dot{V} P(t)=\dot{x}_{1}(t)=\dot{E}(t) *\left(L V V(t)-V_{0}\right)+E(t) *\left(-\frac{1}{R_{a}} * x_{1}(t)+\frac{1}{R_{a}} * x_{4}(t)\right) \\
\dot{x}_{1}(t)=\left(\frac{\dot{E}(t)}{E(t)}-\frac{E(t)}{R_{a}}\right) * x_{1}(t)+\frac{E(t)}{R_{a}} * x_{4}(t)
\end{gathered}
$$

To solve for $\dot{x}_{2}(t)$, we apply KVL to the outer loop of the circuit shown in Figure 3.10 using $i_{R_{p}}=C_{2} \frac{d x_{2}}{d t}=C_{2} * \dot{x}_{2}$. This yields:

$$
\dot{x}_{2}(t)=\frac{-1}{R_{p} C_{2}} * x_{2}(t)+\frac{1}{R_{p} C_{2}} * x_{3}(t)
$$

To solve for $\dot{x}_{3}(t)$, we also apply KVL to the same loop, but using $i_{R_{p}}=x_{5}-C_{3} * \dot{x}_{3}$ from equation (3.20), we obtain:

$$
x_{2}-x_{3}+R_{p} *\left(x_{5}-C_{3} * \dot{x}_{3}\right)=0
$$

which yields:

$$
\dot{x}_{3}(t)=\frac{1}{R_{p} C_{3}} * x_{2}(t)-\frac{1}{R_{p} C_{3}} * x_{3}(t)+\frac{1}{C_{3}} * x_{5}(t)
$$


To solve for $\dot{x}_{4}(t)$, we apply KCL at the node joining the following circuit components: $R_{a}, R_{c}$, and $C_{4}$ in Figure 3.10, we get:

$$
\frac{x_{1}(t)-x_{4}(t)}{R_{a}}=C_{4} * \dot{x}_{4}(t)+x_{5}(t)
$$

which can be re-arranged as follows:

$$
\dot{x}_{4}(t)=\frac{1}{R_{a} C_{4}} * x_{1}(t)-\frac{1}{R_{a} C_{4}} * x_{4}(t)-\frac{1}{C_{4}} * x_{5}(t)
$$

To solve for $\dot{x}_{5}(t)$, we apply KVL to the inner loop of the circuit shown in Figure 3.10. It is important to remember that the voltage across an inductor " $L$ " follows the equation below:

$$
v=L \frac{d i}{d t}
$$

thus, applying KVL yields:

$$
-x_{4}(t)+R_{c} * x_{5}(t)+L * \dot{x}_{5}(t)+x_{3}(t)=0
$$

which can be re-written as follows:

$$
\dot{x}_{5}(t)=\frac{-1}{L} * x_{3}(t)+\frac{1}{L} * x_{4}(t)-\frac{R_{C}}{L} * x_{5}(t)
$$


Putting equations (3.22), (3.23), (3.24), (3.25), and (3.27) together to solve for the matrix $A(t)$, where $\dot{x}(t)=A(t) * x(t)$, we obtain:

$$
\left[\begin{array}{c}
\dot{x}_{1}(t) \\
\dot{x}_{2}(t) \\
\dot{x}_{3}(t) \\
\dot{x}_{4}(t) \\
\dot{x}_{5}(t)
\end{array}\right]=\left[\begin{array}{ccccc}
\frac{\dot{E}(t)}{E(t)}-\frac{E(t)}{R_{a}} & 0 & 0 & \frac{E(t)}{R_{a}} & 0 \\
0 & \frac{-1}{R_{p} C_{2}} & \frac{1}{R_{p} C_{2}} & 0 & 0 \\
0 & \frac{1}{R_{p} C_{3}} & \frac{-1}{R_{p} C_{3}} & 0 & \frac{1}{C_{3}} \\
\frac{1}{R_{a} C_{4}} & 0 & 0 & \frac{-1}{R_{a} C_{4}} & \frac{-1}{C_{4}} \\
0 & 0 & \frac{-1}{L} & \frac{1}{L} & \frac{-R_{c}}{L}
\end{array}\right] *\left[\begin{array}{l}
x_{1}(t) \\
x_{2}(t) \\
x_{3}(t) \\
x_{4}(t) \\
x_{5}(t)
\end{array}\right]
$$

Thus, $A_{2}(t)$, where the subscript " 2 " stands for the ejection phase, can be written as:

$$
A_{2}(t)=\left[\begin{array}{ccccc}
\frac{\dot{E}(t)}{E(t)}-\frac{E(t)}{R_{a}} & 0 & 0 & \frac{E(t)}{R_{a}} & 0 \\
0 & \frac{-1}{R_{p} C_{2}} & \frac{1}{R_{p} C_{2}} & 0 & 0 \\
0 & \frac{1}{R_{p} C_{3}} & \frac{-1}{R_{p} C_{3}} & 0 & \frac{1}{C_{3}} \\
\frac{1}{R_{a} C_{4}} & 0 & 0 & \frac{-1}{R_{a} C_{4}} & \frac{-1}{C_{4}} \\
0 & 0 & \frac{-1}{L} & \frac{1}{L} & \frac{-R_{c}}{L}
\end{array}\right]
$$

\section{$\underline{\text { Isovolumic relaxation phase }}$}

The aortic valve closes when the pressure in the LV falls below that in the aorta, which means that both the mitral and aortic valves are closed again. This is called isovolumic relaxation, a phase that precedes blood filling and represents the onset of diastole. Since both valves are closed, this phase follows the same equations used in the isovolumic contraction phase that was discussed earlier. Thus, the system matrix $A_{3}(t)$ that 
describes the isovolumic relaxation phase, where the subscript " 3 " stands for the isovolumic relaxation phase, is identical to the system matrix $A_{1}(t)$ of the isovolumic contraction phase, according to the following:

$$
A_{3}(t)=A_{1}(t)=\left[\begin{array}{ccccc}
\frac{\dot{E}(t)}{E(t)} & 0 & 0 & 0 & 0 \\
0 & \frac{-1}{R_{p} C_{2}} & \frac{1}{R_{p} C_{2}} & 0 & 0 \\
0 & \frac{1}{R_{p} C_{3}} & \frac{-1}{R_{p} C_{3}} & 0 & \frac{1}{C_{3}} \\
0 & 0 & 0 & 0 & \frac{-1}{C_{4}} \\
0 & 0 & \frac{-1}{L} & \frac{1}{L} & \frac{-R_{c}}{L}
\end{array}\right]
$$

\section{Filling phase}

The filling phase starts when the pressure in the LV falls below the pressure in the LA which is the moment the mitral valve opens to fill the LV with blood. The aortic valve is closed during this phase since the pressure in the LV is below the aortic pressure. Thus, as indicated in Table $3.1, \mathrm{D}_{\mathrm{m}}$ is closed-circuit and $\mathrm{D}_{\mathrm{a}}$ is open-circuit as shown in the equivalent circuit in Figure 3.11 below.

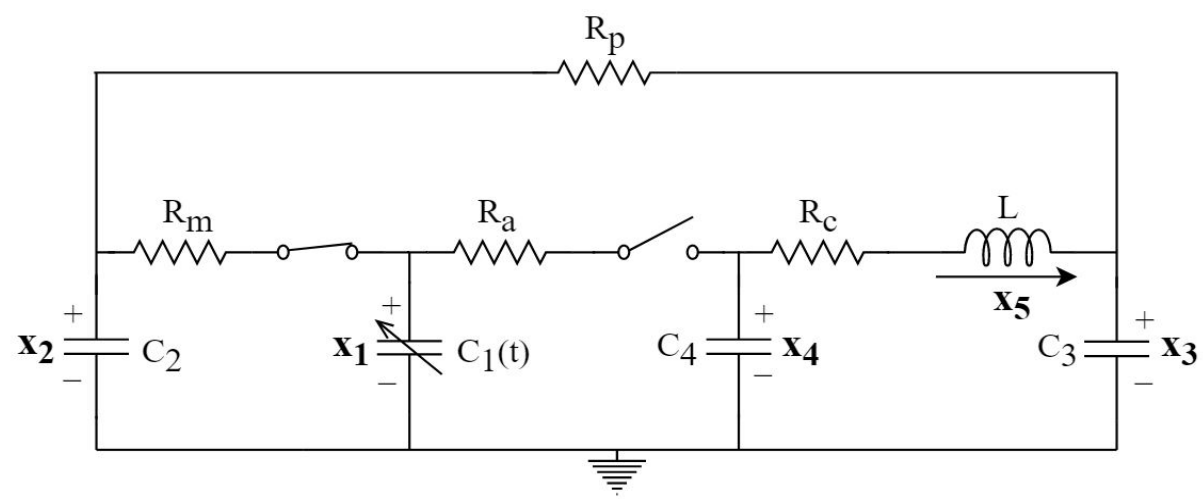

Figure 3.11. Equivalent circuit representing the filling phase. The mitral valve is open (short circuit), but the aortic valve is closed (open circuit). 
Figure 3.11 can be simplified as in the figure below:

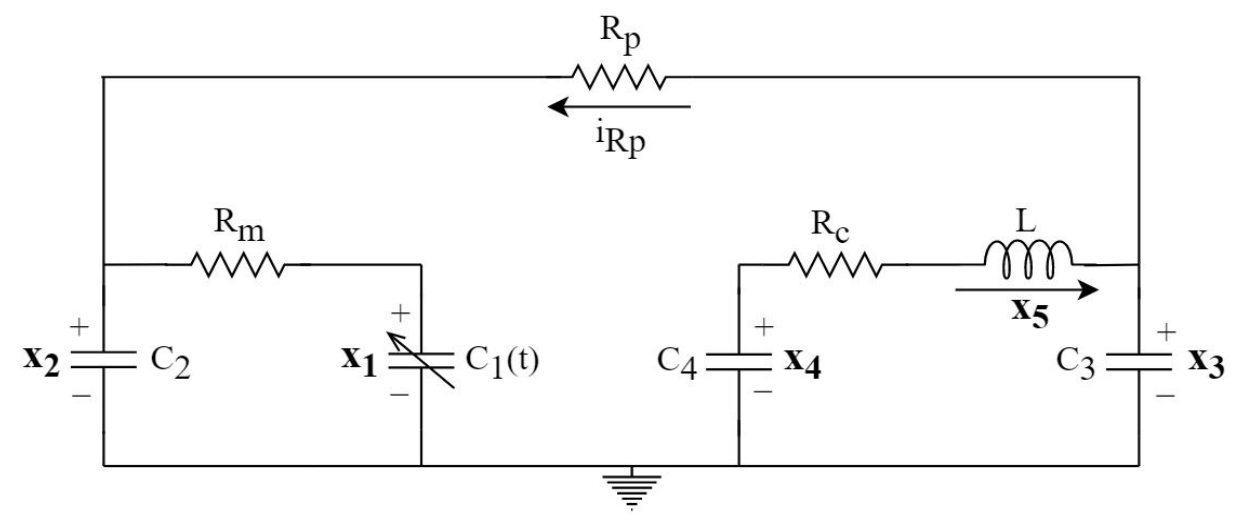

Figure 3.12. Simplified circuit for the filling phase.

To solve for $\dot{x}_{1}$, we again use equation (3.12) as shown below:

$$
L \dot{V} P(t)=\dot{x}_{1}(t)=\dot{E}(t) *\left(L V V(t)-V_{0}\right)+E(t) * L \dot{V} V(t)
$$

$L \dot{V} V(t)$ here equals the blood flow through the mitral valve, which follows the pressure drop between the left atrium (LAP) and left ventricle (LVP) according to the following:

$$
L \dot{V} V(t)=Q_{\text {in }}-Q_{\text {out }}=\frac{x_{2}(t)-x_{1}(t)}{R_{m}}-0=\frac{x_{2}(t)-x_{1}(t)}{R_{m}}
$$

This yields:

$$
\begin{gathered}
L \dot{V} P(t)=\dot{x}_{1}(t)=\dot{E}(t) *\left(L V V(t)-V_{0}\right)+E(t) *\left(\frac{x_{2}(t)-x_{1}(t)}{R_{m}}\right) \\
\dot{x}_{1}(t)=\left(\frac{\dot{E}(t)}{E(t)}-\frac{E(t)}{R_{m}}\right) * x_{1}(t)+\frac{E(t)}{R_{m}} * x_{2}(t)
\end{gathered}
$$

The current flowing through the resistor $R_{p}$ in the direction shown in Figure 3.12 is:

$$
i_{R_{p}}=x_{5}-C_{3} * \dot{x}_{3}=C_{2} * \dot{x}_{2}+\frac{x_{2}(t)-x_{1}(t)}{R_{m}}
$$


Applying KVL to the outer loop of the circuit in Figure 3.12 to solve for $\dot{x}_{2}$, we get:

$$
x_{2}-x_{3}+R_{p} *\left(C_{2} * \dot{x}_{2}+\frac{x_{2}(t)-x_{1}(t)}{R_{m}}\right)=0
$$

which can be re-arranged according to the following:

$$
\dot{x}_{2}(t)=\frac{1}{R_{m} C_{2}} * x_{1}(t)-\frac{\left(R_{p}+R_{m}\right)}{R_{p} R_{m} C_{2}} * x_{2}(t)+\frac{1}{R_{p} C_{2}} * x_{3}(t)
$$

Applying KVL to the same loop again but using the other definition for the current flowing through $R_{p}$ as in equation (3.33), we get:

$$
\begin{gathered}
x_{2}-x_{3}+R_{p} *\left(x_{5}-C_{3} * \dot{x}_{3}\right)=0 \\
\dot{x}_{3}(t)=\frac{1}{R_{p} C_{3}} * x_{2}(t)-\frac{1}{R_{p} C_{3}} * x_{3}(t)+\frac{1}{C_{3}} * x_{5}(t)
\end{gathered}
$$

We can easily derive the equation for $\dot{x}_{4}$ from Figure 3.12 according to the following:

$$
\dot{x}_{4}(t)=\frac{-1}{C_{4}} * x_{5}(t)
$$

To solve for $\dot{x}_{5}$, we apply Kirchhoff's voltage law (KVL) on the inner loop of the circuit shown in Figure 3.12, we get $-x_{4}+R_{c} * x_{5}+L * \dot{x}_{5}+x_{3}=0$. This can be re-written as shown below:

$$
\dot{x}_{5}(t)=\frac{-1}{L} * x_{3}(t)+\frac{1}{L} * x_{4}(t)-\frac{R_{C}}{L} * x_{5}(t)
$$


Putting equations (3.32), (3.34), (3.35), (3.36), and (3.37) together to solve for the state matrix $A(t)$ of the system $\dot{x}(t)=A(t) * x(t)$, we obtain:

$$
\left[\begin{array}{l}
\dot{x}_{1}(t) \\
\dot{x}_{2}(t) \\
\dot{x}_{3}(t) \\
\dot{x}_{4}(t) \\
\dot{x}_{5}(t)
\end{array}\right]=\left[\begin{array}{ccccc}
\frac{\dot{E}(t)}{E(t)}-\frac{E(t)}{R_{m}} & \frac{E(t)}{R_{m}} & 0 & 0 & 0 \\
\frac{1}{R_{m} C_{2}} & -\frac{\left(R_{p}+R_{m}\right)}{R_{p} R_{m} C_{2}} & \frac{1}{R_{p} C_{2}} & 0 & 0 \\
0 & \frac{1}{R_{p} C_{3}} & \frac{-1}{R_{p} C_{3}} & 0 & \frac{1}{C_{3}} \\
0 & 0 & 0 & 0 & \frac{-1}{C_{4}} \\
0 & 0 & \frac{-1}{L} & \frac{1}{L} & \frac{-R_{c}}{L}
\end{array}\right] *\left[\begin{array}{l}
x_{1}(t) \\
x_{2}(t) \\
x_{3}(t) \\
x_{4}(t) \\
x_{5}(t)
\end{array}\right]
$$

Thus, $A_{4}(t)$, where the subscript " 4 " stands for the filling phase, can be written as:

$$
A_{4}(t)=\left[\begin{array}{ccccc}
\frac{\dot{E}(t)}{E(t)}-\frac{E(t)}{R_{m}} & \frac{E(t)}{R_{m}} & 0 & 0 & 0 \\
\frac{1}{R_{m} C_{2}} & -\frac{\left(R_{p}+R_{m}\right)}{R_{p} R_{m} C_{2}} & \frac{1}{R_{p} C_{2}} & 0 & 0 \\
0 & \frac{1}{R_{p} C_{3}} & \frac{-1}{R_{p} C_{3}} & 0 & \frac{1}{C_{3}} \\
0 & 0 & 0 & 0 & \frac{-1}{C_{4}} \\
0 & 0 & \frac{-1}{L} & \frac{1}{L} & \frac{-R_{c}}{L}
\end{array}\right]
$$




\subsection{Model Validation}

One way to validate the cardiovascular model presented in Figure 3.6 is by simulating it and checking if it can reproduce expected hemodynamic data. Another way to validate the model is by varying preload (such as mitral valve resistance) or afterload (such as systemic vascular resistance) conditions and checking whether the model can behave as expected by showing a linear end-systolic pressure-volume relationship (ESPVR) [61]. The two methods for validating the cardiovascular model illustrated in Figure 3.6 will be discussed next.

\subsubsection{Model Validation by Reproducing Expected Hemodynamics}

The cardiovascular model presented in Figure 3.6 was simulated in MATLAB to reproduce close-to-normal clinical hemodynamics using the following values: heart rate 75 beats $/ \mathrm{min}, E_{\max }=2.0 \mathrm{mmHg} / \mathrm{ml}, E_{\min }=0.05 \mathrm{mmHg} / \mathrm{ml}$, and $V_{0}=10 \mathrm{ml}$. The parametric values of the hemodynamical elements used to simulate the cardiovascular model are shown in Table 3.3. The resulting waveforms of the left ventricular pressure LVP, left atrial pressure LAP, aortic pressure AoP, aortic flow $\mathrm{Q}_{\mathrm{A}}$, left ventricular volume LVV, and PV-loop are shown in Figure 3.13. The simulations resulted the following hemodynamics: systolic and diastolic aortic pressure of 120.2/ 79.6 $\mathrm{mm} \mathrm{Hg}$, mean arterial pressure (MAP) of $101.7 \mathrm{mmHg}$, stroke volume of $71.3 \mathrm{ml} /$ beat, cardiac output of 5.34 $l / \mathrm{min}$, and end-diastolic volume (EDV) of $139.7 \mathrm{ml}$. These numbers are within normal hemodynamical ranges [62], which validate the use of this model. 


\begin{tabular}{|c|c|c|}
\hline Parameters & Physiological meaning & Value (unit) \\
\hline $\mathrm{R}_{\mathrm{p}}$ & Peripheral resistance & $1.0(\mathrm{mmHg} \mathrm{s} / \mathrm{ml})$ \\
\hline $\mathrm{R}_{\mathrm{m}}$ & Mitral valve resistance & $0.005(\mathrm{mmHg} \mathrm{s} / \mathrm{ml})$ \\
\hline $\mathrm{R}_{\mathrm{a}}$ & Aortic valve resistance & $0.001(\mathrm{mmHg} \mathrm{s} / \mathrm{ml})$ \\
\hline $\mathrm{R}_{\mathrm{c}}$ & Characteristic impedance & $0.04(\mathrm{mmHg} \mathrm{s} / \mathrm{ml})$ \\
\hline $\mathrm{C}_{1}(\mathrm{t})$ & Time-varying compliance of $\mathrm{LV}$ & Reciprocal of elastance E(t) \\
\hline $\mathrm{C}_{2}$ & Left atrial compliance & $4.4(\mathrm{ml} / \mathrm{mmHg})$ \\
\hline $\mathrm{C}_{3}$ & Arterial compliance & $1.33(\mathrm{ml} / \mathrm{mmHg})$ \\
\hline $\mathrm{C}_{4}$ & Aortic compliance & $0.08(\mathrm{ml} / \mathrm{mmHg})$ \\
\hline $\mathrm{L}$ & Inertance & $0.0005(\mathrm{mmHg} \mathrm{s} / \mathrm{ml})$ \\
\hline
\end{tabular}

Table 3.3. Parametric values of the hemodynamical elements used in the cardiovascular model [61].
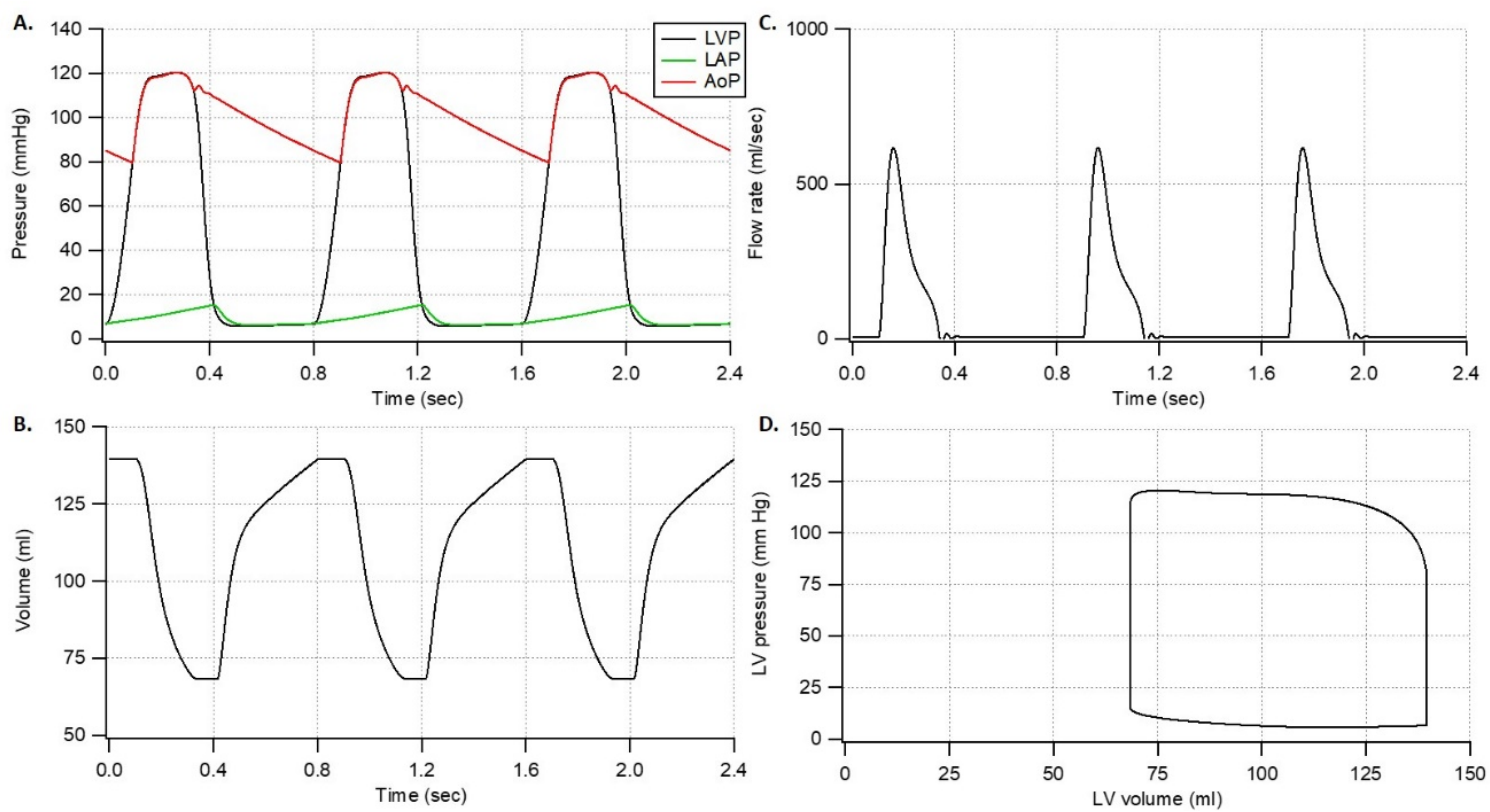

Figure 3.13. Waveforms of the hemodynamics resulting from the simulation of the cardiovascular model that represents a normal (healthy) condition. Pressure waveforms of the left ventricle LVP, left atrium LAP, and arteries AP are shown in (A). Left ventricular volume LVV is shown in (B). Blood flow in the aorta $\mathrm{Q}_{\mathrm{A}}$ is shown in (C). The resulting left ventricular pressure-volume relationship, PV-loop, is shown in (D). 


\subsubsection{Model Validation by Varying Preload and Afterload Conditions}

Another way to validate the cardiovascular model presented in Figure 3.6 is by varying preload and afterload conditions and checking whether the model shows expected behavior by reproducing a linear end-systolic pressure-volume relationship (ESPVR). Different preload conditions can be simulated by varying the mitral valve resistance, while afterload conditions can be simulated by varying the systemic vascular resistance. Here, we show the simulation results of varying the systemic vascular resistance (similar simulations for different preload conditions by varying the mitral valve resistance, results not shown, were done and showed a similar behavior). The PV-loops resulting from the simulations of three different values for the systemic vascular resistance $\left(R_{p}=0.5, R_{p}=\right.$ 1.5 , and $R_{p}=2.0$ ), in addition to the original value $R_{p}=1.0$, which represents the normal healthy condition, were all bounded by the same ESPVR line as shown in Figure 3.14. The left ventricular parameters were all kept unchanged when simulating these different afterload conditions, $\mathrm{E}_{\min }=0.05 \mathrm{mmHg} / \mathrm{ml}, \mathrm{E}_{\max }=2.0 \mathrm{mmHg} / \mathrm{ma}, \mathrm{V}_{0}=10 \mathrm{ml}$. In addition, end-diastolic volume (EDV) was also kept unchanged in all simulations. A comparison of the hemodynamics resulting from the simulation of the different afterload conditions is summarized in Table 3.4. 


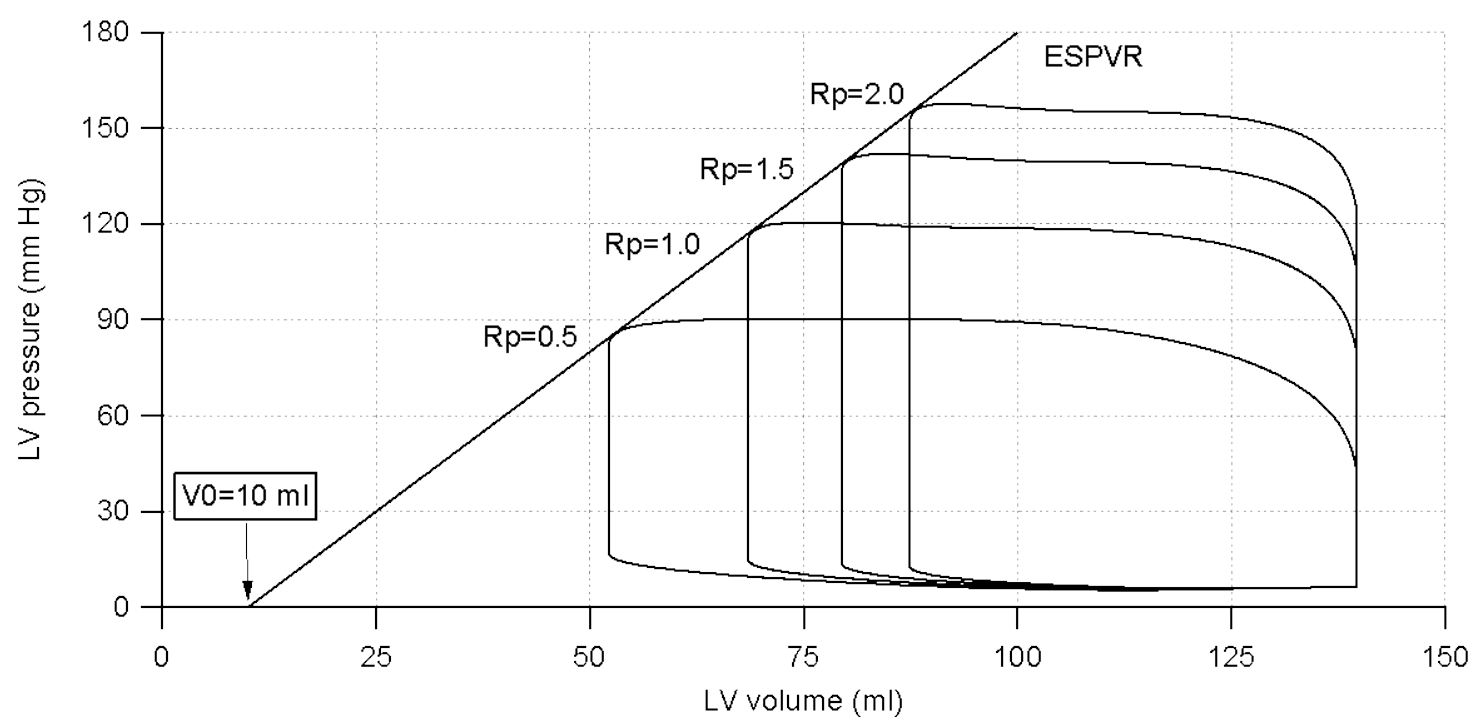

Figure 3.14. PV-loops for different systemic vascular resistance values (different afterload conditions). The PV-loops were all bounded from the upper left corner by the same ESPVR line, the slope of which representing $\mathrm{E}_{\max }$, or end-systolic elastance Ees.

\begin{tabular}{|c|c|c|c|c|c|}
\hline $\begin{array}{c}\text { Systemic resistance value } \\
(\mathrm{mmHg} / \mathrm{ml})\end{array}$ & $\begin{array}{c}\mathbf{P}_{\mathbf{s}} \\
(\mathrm{mm} \mathrm{Hg})\end{array}$ & $\begin{array}{c}\mathbf{P}_{\mathbf{d}} \\
(\mathrm{mm} \mathrm{Hg})\end{array}$ & $\begin{array}{c}\text { MAP } \\
(\mathrm{mm} \mathrm{Hg})\end{array}$ & $\begin{array}{c}\mathbf{C O} \\
(\mathrm{l} / \mathrm{min})\end{array}$ & $\begin{array}{c}\mathbf{S V} \\
(\mathrm{ml} / \mathrm{beat})\end{array}$ \\
\hline $\mathrm{R}_{\mathrm{p}}=0.5$ & 89.9 & 43.1 & 68.5 & 6.56 & 87.5 \\
\hline $\mathrm{R}_{\mathrm{p}}=1.0$ & 120.2 & 79.6 & 101.7 & 5.34 & 71.3 \\
\hline $\mathrm{R}_{\mathrm{p}}=1.5$ & 141.7 & 105.6 & 124.8 & 4.52 & 60.3 \\
\hline $\mathrm{R}_{\mathrm{p}}=2.0$ & 157.4 & 124.8 & 141.8 & 3.92 & 52.3 \\
\hline
\end{tabular}

Table 3.4. Summary of hemodynamics resulting from simulating the cardiovascular model with different afterload conditions (different systemic vascular resistance values). $\mathrm{P}_{\mathrm{s}}$ is systolic pressure, $\mathrm{P}_{\mathrm{d}}$ is diastolic pressure, MAP is mean arterial pressure, $\mathrm{CO}$ is cardiac output, and $\mathrm{SV}$ is stroke volume.

\subsubsection{Simulating Unhealthy Condition}

After validating the cardiovascular model by reproducing normal hemodynamic data when using normal parametric values for total peripheral resistance and arterial compliance (Table 3.3), we simulated hypertension by inducing an unhealthy condition. 
This was achieved by increasing the total peripheral resistance by $60 \%$ and decreasing the arterial compliance by $45 \%$ while keeping all other parameters unchanged, including heart rate. The resulting hemodynamic data are: systolic and diastolic pressures of 149.8 and $94.1 \mathrm{mmHg}$ respectively, mean arterial pressure of $123.3 \mathrm{mmHg}$, stroke volume of 56 $\mathrm{ml} / \mathrm{beat}$, cardiac output of $4.2 \mathrm{l} / \mathrm{min}$, and end-diastolic volume of $139.7 \mathrm{ml}$. We can notice an increase in all pressure readings, compared to the results of healthy condition, associated with a decrease in stroke volume and cardiac output. The corresponding waveforms for LVP, LAP, AoP, QA, LVV, and PV-loop are presented in Figure 3.15.
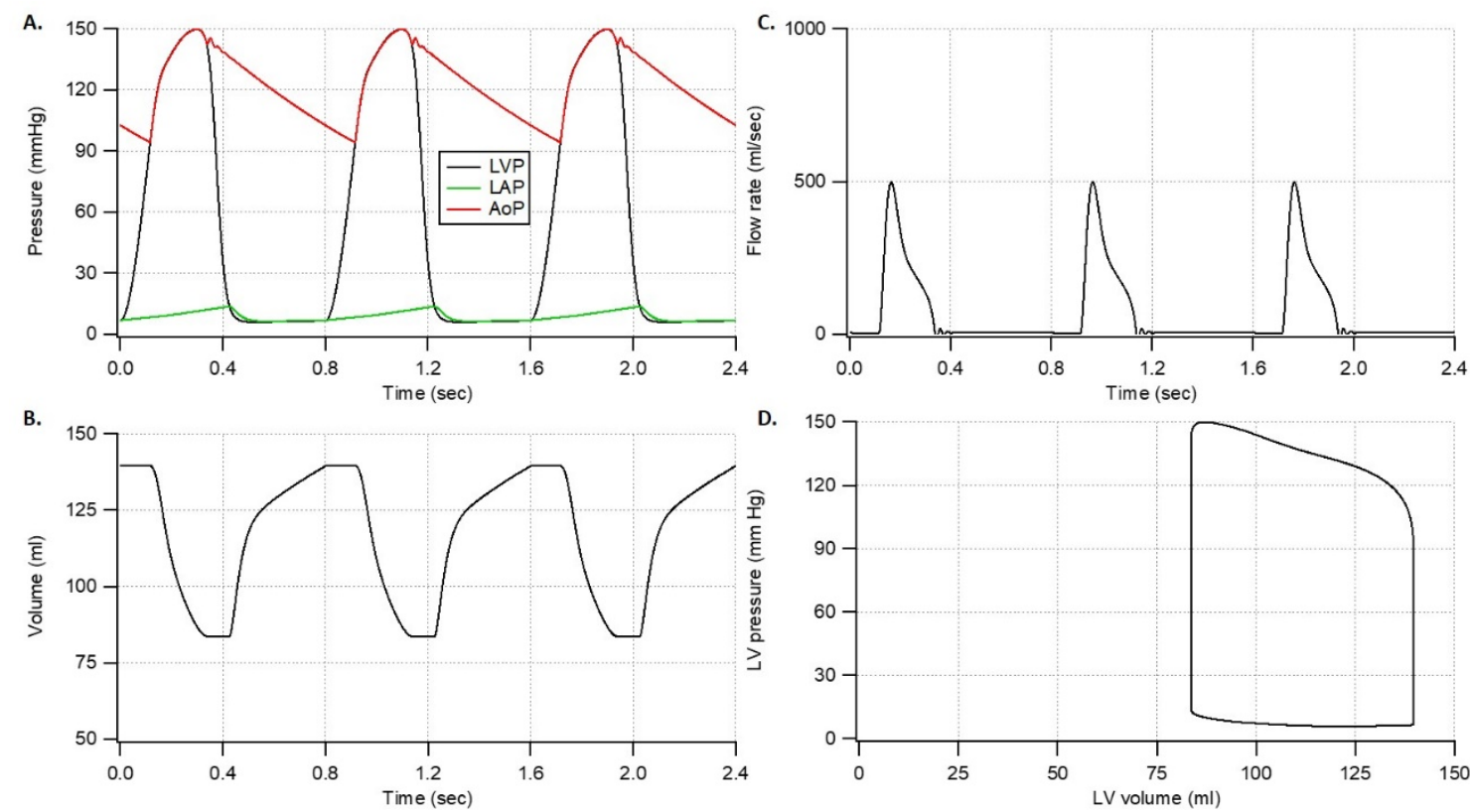

Figure 3.15. Waveforms of the hemodynamics resulting from simulating the cardiovascular model of induced unhealthy condition representing hypertension. Pressure waveforms of the left ventricle LVP, left atrium LAP, and arteries AP are shown in (A). Left ventricular volume LVV is shown in (B). Blood flow in the aorta $\mathrm{Q}_{\mathrm{A}}$ is shown in $(\mathrm{C})$. The resulting left ventricular pressure-volume relationship, PV-loop, is shown in (D). 


\subsection{Preliminary Studies for Treating RH}

Our preliminary studies to demonstrate opportunities to improve the high blood pressure in the unhealthy, hypertensive case included two methods. The first one utilizes the cardiac contractility modulation technique by showing the effect of decreasing the contractility force of the LV on blood pressure. The decrease in contractile force is achieved by decreasing the end-systolic elastance $\left(E_{e s}\right)$ or $E_{\max }$ which represents the measurement index for contractility $[63,64]$. The second method utilizes the addition of artificial compliance, as an attempt to increase the arterial compliance. The preliminary investigations for these two methods are discussed next.

\subsubsection{Using CCM to Decrease the Cardiac Contractility}

As discussed earlier, application of cardiac contractility modulation (CCM) signals can modulate the contractility force of the heart muscle. Studies have investigated the application of both positive and negative CCM signals and concluded that positive current signals enhance contractility and increase maximum $d P / d t$, while that negative signals show the opposite effect. A direct way to simulate the change in contractility is by altering the end-systolic elastance $E_{e s}$, which represents the maximum value $\left(E_{\max }\right)$ in the timevarying elastance function (Figure 3.4) that was used to model the left ventricle in our cardiovascular model (Figure 3.6). Increasing the value of $E_{\max }$ represents a case of stronger contraction, while decreasing $\mathrm{E}_{\max }$ represents a weaker heart contraction. In our analysis, we investigate the effect of decreasing the contractile force since our goal is to decrease the blood pressure; however, this is expected to be associated with some tradeoffs as we may end up with a lesser cardiac output due to expected weaker blood ejection. The results 
of different simulations to improve blood pressure using different intensity levels of negative CCM signals are demonstrated in Table 3.5. The column denoted "baseline" represents the unhealthy case of hypertension that we want to treat, while the other columns to the right simulate the effect of applying negative CCM signals of different intensities. This effect of weaker contractions is characterized by a $10 \%$ gradual decrement of the initial contractility index $E_{\max }$ that is used in the unhealthy (baseline) condition, where the right most column represents the weakest contractility with $\mathrm{E}_{\max }$ being decreased to $50 \%$ of its original value in the baseline case. Table 3.5 clearly shows that as the contractility index $E_{\max }$ is decreased, all the blood pressure readings improved showing a noticeable decrease; however, this is also associated with a significant decrease in the cardiac output.

\begin{tabular}{l|c|c|c|c|c|c}
\hline Hemodynamic variables & Baseline & $\mathbf{9 0 \%} \mathbf{E}_{\max }$ & $\mathbf{8 0 \%} \mathbf{E}_{\max }$ & $\mathbf{7 0 \%}_{\max }$ & $\mathbf{6 0 \%}_{\max }$ & $\mathbf{5 0 \%}_{\mathbf{m a x}}$ \\
\hline Systolic pressure (mmHg) & 149.8 & 143.9 & 137 & 129.2 & 120 & 109 \\
\hline Diastolic pressure (mmHg) & 94.1 & 90.3 & 86 & 81.1 & 75.3 & 68.6 \\
\hline MAP (mm Hg) & 123.3 & 118.2 & 112.4 & 105.7 & 98 & 88.8 \\
\hline SV (ml) & 56 & 53.5 & 50.6 & 47.3 & 43.5 & 39 \\
\hline CO (1/min) & 4.2 & 4 & 3.8 & 3.55 & 3.3 & 2.9 \\
\hline
\end{tabular}

Table 3.5. Effect of applying negative CCM signals. The column corresponding to baseline represents the unhealthy case that we want to treat by improving its blood pressure. The columns to its right represent the effect of applying negative CCM signals with different intensities, where $90 \% \mathrm{E}_{\max }$ means that contractility was decreased by $10 \%$ of its original value in baseline, and $50 \% \mathrm{E}_{\max }$ represents a contractility decrement of $50 \%$ of its original value in baseline.

\section{Decreasing Contractility While Increasing HR}

After investigating the effect of negative CCM signals on depressing contractility and therefore lowering the blood pressure, which was associated with a decrease in cardiac output, we investigated whether alteration of the heart rate, combined with the CCM effect, 
would make any further improvement. In this experiment, we chose the case when $\mathrm{E}_{\max }$ was decreased to $60 \%$ of its original value in baseline, which resulted the following hemodynamics: systolic, diastolic, and mean arterial pressures of 120, 75.3, and $98 \mathrm{~mm} \mathrm{Hg}$ respectively while $\mathrm{CO}$ is $3.3 \mathrm{l} / \mathrm{min}$ as shown in Table 3.5 . We then increased the heart rate from 75 beats/min to 80,90 , and 100 beats/min. The increase in HR from 75 to 100 beats/min increased the $\mathrm{CO}$ by $\sim 7 \%$, while at the same time increased the pressure values, especially the diastolic one, as shown in Table 3.6.

\begin{tabular}{l|c|c|c|c|c}
\hline \multirow{2}{*}{ Hemodynamic variables } & \multicolumn{2}{|c|}{ HR=75 } & \multicolumn{3}{c}{ 60\% E } \\
\cline { 2 - 6 } & Baseline & $\mathbf{6 0 \%} \mathbf{E}_{\max }$ & HR=80 & HR=90 & HR=100 \\
\hline systolic pressure $(\mathrm{mmHg})$ & 149.8 & 120 & 120.3 & 120.8 & 121.3 \\
\hline diastolic pressure $(\mathrm{mmHg})$ & 94.1 & 75.3 & 77.8 & 82 & 85.6 \\
\hline MAP $(\mathrm{mm} \mathrm{Hg})$ & 123.3 & 98 & 99.4 & 101.9 & 103.9 \\
\hline SV $(\mathrm{ml})$ & 56 & 43.5 & 41.5 & 37.9 & 34.8 \\
\hline $\mathrm{CO}(1 / \mathrm{min})$ & 4.2 & 3.26 & 3.32 & 3.41 & 3.48 \\
\hline
\end{tabular}

Table 3.6. Effect of increasing heart rate. HR was increased from 75 beats $/ \mathrm{min}$ to 80 , 90 , and 100 beats $/ \mathrm{min}$. Increasing the heart rate from 75 to 100 beat $/ \mathrm{min}$, while applying negative CCM signals to improve blood pressure, improved (increased) the cardiac output by $\sim 10 \%$ while showing a slight increase in blood pressures.

\subsubsection{Addition of Artificial Compliance}

In this preliminary study, we investigate the effect of adding an artificial compliance, in an attempt to simulate the expected benefit of increasing the total arterial compliance in improving blood pressure. An additional artificial compliance $\left(\mathrm{C}_{5}\right)$ is placed in parallel with the arterial compliance $\left(C_{3}\right)$ as shown in the modified circuit of the cardiovascular model in Figure 3.16. The overall arterial compliance is then equal to the addition of the two individual compliances $\mathrm{C}_{3}$ and $\mathrm{C}_{5}$. Table 3.7 shows the resulting 
hemodynamics that correspond to the simulations of adding different values for the artificial compliance $\mathrm{C}_{5}$.

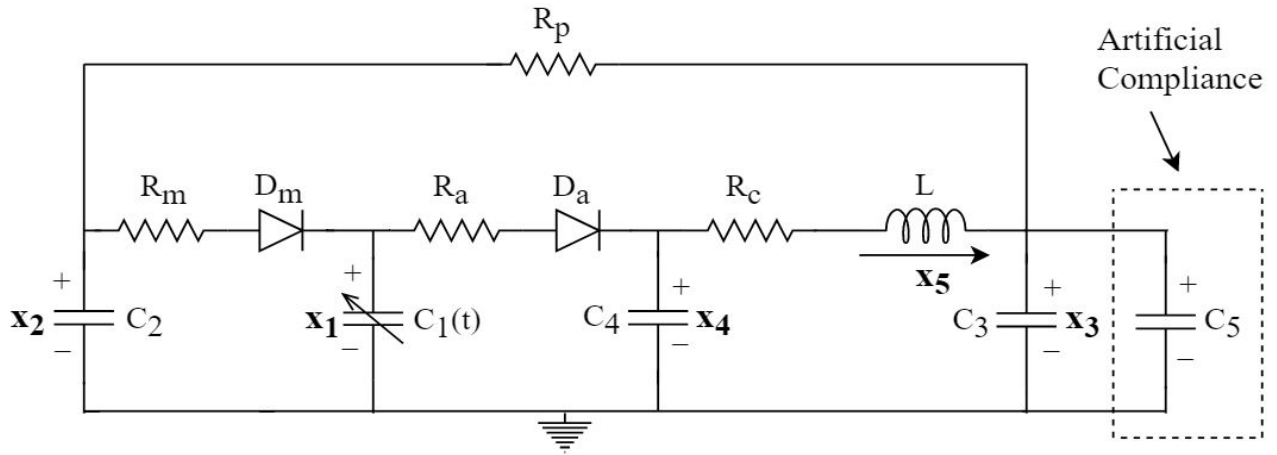

Figure 3.16. Cardiovascular model with the addition of artificial compliance $\mathrm{C}_{4}$.

\begin{tabular}{|c|c|c|c|c|c|c|}
\hline \multirow[b]{2}{*}{ Hemodynamic variables } & \multirow[b]{2}{*}{ Baseline } & \multicolumn{5}{|c|}{$\begin{array}{l}\text { Addition of different values for the artificial } \\
\text { compliance } C_{5}\end{array}$} \\
\hline & & $\begin{array}{l}20 \% \\
\text { of } \mathrm{C}_{3}\end{array}$ & $\begin{array}{l}40 \% \\
\text { of } \mathrm{C}_{3}\end{array}$ & $\begin{array}{l}60 \% \\
\text { of } \mathrm{C}_{3}\end{array}$ & $\begin{array}{l}80 \% \\
\text { of } \mathrm{C}_{3}\end{array}$ & $\begin{array}{l}100 \% \\
\text { of } \mathrm{C}_{3}\end{array}$ \\
\hline Systolic pressure (mmHg) & 149.8 & 143.9 & 139 & 135 & 131.5 & 128.5 \\
\hline Diastolic pressure $(\mathrm{mmHg})$ & 94.1 & 96.5 & 97.7 & 98.4 & 98.6 & 98.5 \\
\hline MAP (mm Hg) & 123.3 & 121.4 & 119.6 & 117.7 & 116 & 114.4 \\
\hline $\mathrm{SV}(\mathrm{ml})$ & 56 & 55.2 & 54.4 & 53.5 & 52.8 & 52 \\
\hline $\mathrm{CO}(1 / \mathrm{min})$ & 4.2 & 4.14 & 4.08 & 4 & 3.96 & 3.9 \\
\hline
\end{tabular}

Table 3.7. Effect of the addition of artificial compliance. Baseline represents the unhealthy case to be treated. The columns to its right represent additions of artificial compliance. $20 \%$ of $\mathrm{C}_{3}$ means an addition of an artificial compliance of a value equals $20 \%$ of the arterial compliance $\mathrm{C}_{3}$, while the right most column represents the addition of the largest artificial compliance of a value equals $\mathrm{C}_{3}$.

The columns in Table 3.7 compare the baseline, unhealthy condition, with cases showing the addition of an artificial compliance of different values. Each column to the right of the baseline represents a $20 \%$ gradual increment in the value of the added artificial compliance 
compared to the one on its left. The right most column corresponds to an addition of artificial compliance of a value that is equal to that of the arterial compliance $\mathrm{C}_{3}$, which represents the highest value for the added artificial compliance in this comparison. The table shows that the addition of artificial compliance decreases (improves) systolic and mean arterial pressures; however, it increases the diastolic pressure. On the other hand, the addition of artificial compliance has slightly decreased the cardiac output.

\subsection{Discussion}

A lumped-parameter electric circuit model describing the cardiovascular system was presented (Figure 3.6) and validated (Figure 3.13 and Figure 3.14). The model successfully reproduced expected hemodynamics, such as blood pressure and flow. We then simulated hypertension by inducing an unhealthy condition by increasing the systemic vascular resistance by $60 \%$ and decreasing the arterial compliance by $45 \%$ (Figure 3.15 ).

Our preliminary analysis for treating the induced hypertensive case included two studies. The first study involved changing the contraction kinematics of the left ventricle by decreasing the contractile force via negative amplitude current CCM signals. The effect of CCM signals showed an improvement (decrease) in blood pressure; however, this was associated with a decrease in cardiac output (Table 3.5). Decreasing the contractile force by $40 \%$ (i.e. to $60 \%$ of $\mathrm{E}_{\max }$ ) caused a decrease in blood pressure from $149.8 / 94.1 \mathrm{mmHg}$, in the "induced" unhealthy condition, to $120 / 75.3 \mathrm{mmHg}$; however, this was associated with a decrease in cardiac output by $\sim 21 \%$. We then investigated the effect of increasing the heart rate from 75 beats/min to 100 beats/min while applying the CCM signals and showed that this increased (improved) the $\mathrm{CO}$ by $\sim 7 \%$ (Table 3.6). The second preliminary 
study investigated the addition of artificial compliance which showed a significant improvement in lowering the systolic pressure and mean arterial pressure; however, diastolic pressure was slightly increased, while that cardiac output was minimally decreased (Table 3.7).

The cardiovascular model presented in this chapter showed to be a reasonable testbed for our research study. The two methods investigated in our preliminary analysis for treating resistant hypertension give an indication that hypertension could be controlled. LVADs have shown to be a promising technology as a long-term treatment option for heart failure patients, and we anticipate that such technology could be a feasible candidate for treating hypertension. This is simply because LVADs can control the flow of blood circulation via its controlled pump. However, the use of an LVAD with an intact heart does not seem to be a preferred treatment option for $\mathrm{RH}$ since it may disable the functionality of the intact left ventricle.

In the proceeding chapters, we introduce a novel approach for treating resistant hypertension. An accumulator device idea is implemented with the cardiovascular model that is presented in Figure 3.6. The working concept of this accumulator device and its influence on blood pressure waveforms, especially the aortic pressure, will be discussed next. 


\section{CHAPTER 4 Introducing the Accumulator Concept for Treating RH}

\subsection{Introduction}

Most hypertension treatment methods are based on reducing total peripheral resistance through lifestyle changes and drug treatment. However, RH patients, as mentioned earlier, do not respond to such pharmacological therapy. With the fact there is no control over the peripheral resistance for such class of patients, our focus is targeting the pumping action of the heart. Thus, in this work, we investigate to mechanically alter the way the blood is pumped into the aorta such that to reduce the systolic pressure, without reducing the cardiac output.

In this research study, we introduce the novel idea of implementing what we called the accumulator device and hope that such idea will serve as a successful candidate for treating $\mathrm{RH}$ in the future. This accumulator device is meant to regulate blood ejection into the aorta in such a way that systolic pressure is reduced, without reducing the CO. This is achieved by accumulating a partial amount of the blood pumped by the LV during systole and discharging it back to the aorta during diastole, thus providing another route between the LV and aorta. This new approach showed to reduce the systolic pressure, with the level of reduction in systolic pressure being dependent on the amount of blood accumulated during the ejection phase of the cardiac cycle. The reduction in systolic pressure is associated with an increase in diastolic pressure. This is intuitive since we are maintaining the $\mathrm{CO}$ in an unaltered state and that the peripheral resistance of the body is not changed, which should result in the same MAP. 
To the best of our knowledge, there is no existing treatment approach in the literature that treats hypertension by altering the way in which the heart pumps blood into the aorta. This new approach showed a significant improvement in reducing the systolic pressure, a finding that may be of interest to researchers for further investigations.

\subsection{Description}

The proposed accumulator device is illustrated by the basic schematic shown in Figure 4.1. The device comprises a chamber of a spring-loaded piston accumulator for blood accumulation, inlet and outlet ports, each associated with a unidirectional check valve to restrict the direction of blood flow, and additionally, a force source that is applied on the piston during discharging to control the blood discharging rate. The accumulator inlet is connected to the LV to allow for blood flow into the accumulator, and the outlet is connected to the aorta to allow for blood discharging back to the aorta, in a similar fashion to that of an LVAD.

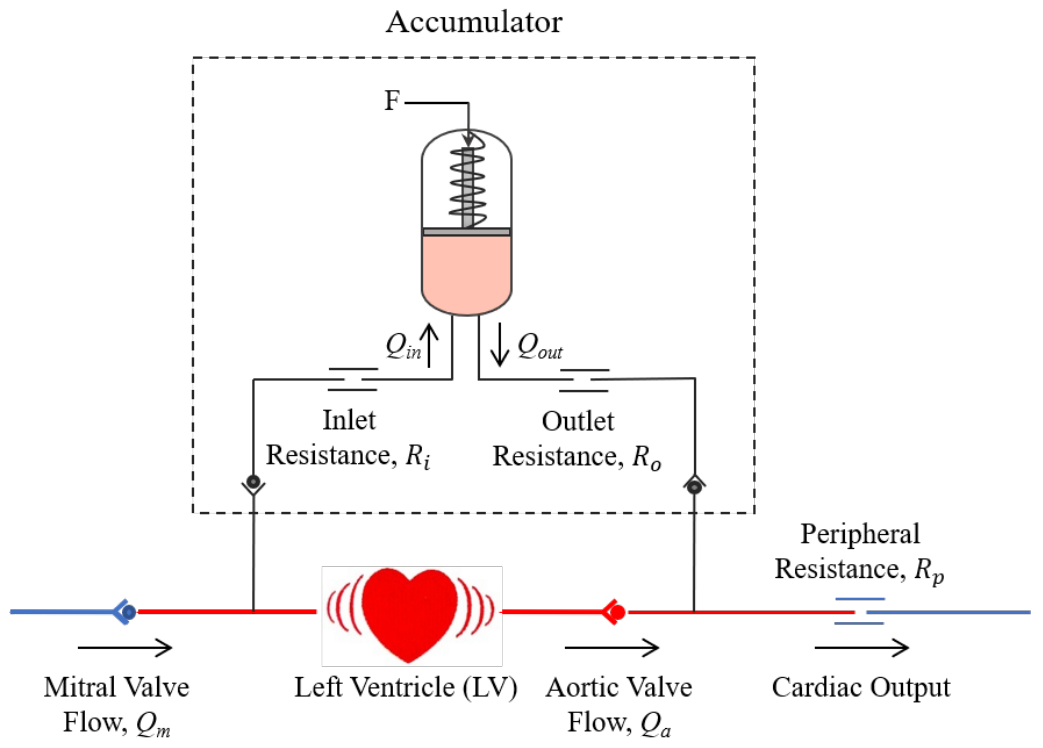

Figure 4.1. A schematic diagram illustrating the accumulator device concept. 
The electric circuit element that is analogous to the hydraulic accumulator is the capacitor as illustrated in Figure 4.2. A schematic describing the implementation of the accumulator device into the lumped parameter model of the cardiovascular system (Figure 3.6) is illustrated in Figure 4.3. Check valves are represented by switches, and the controlled force that is applied on the piston to control the blood flow rate is represented by the variable voltage source $U(t)$.

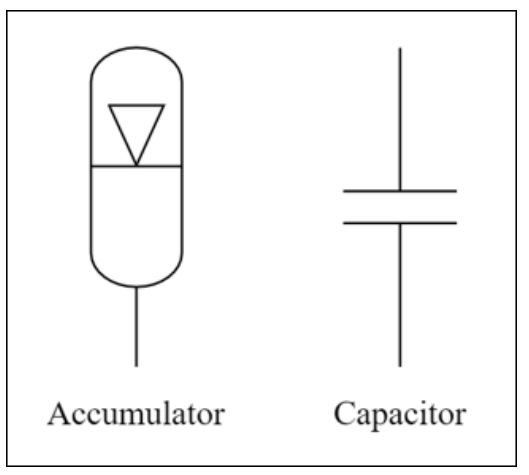

Figure 4.2. Analogy between hydraulic accumulator and electric capacitor.

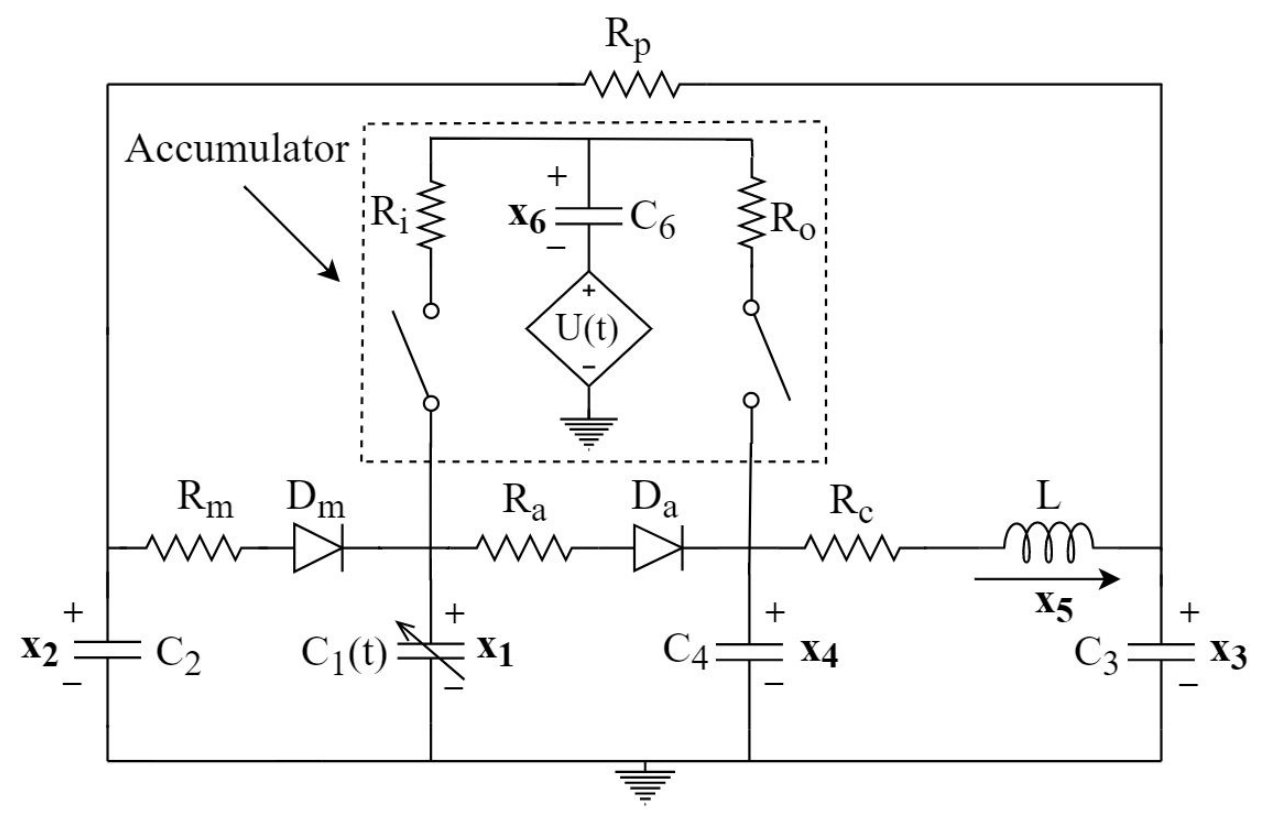

Figure 4.3. Implementation of the accumulator concept into the cardiovascular system lumped model. 
The check valve of the accumulator inlet is supposed to be open only during accumulation to allow for blood flow from the left ventricle to the accumulator, while that the other check valve (of the outlet) is closed. To discharge the accumulated blood back into the aorta, the check valve of the accumulator outlet should be open while that the inlet valve is closed. Accumulator filling occurs during the ejection phase when the aortic valve is open, which means that the blood that is pumped out of the left ventricle during systole has two routes: a natural route through the aortic valve and another route through the inlet of the accumulator device in order to fill the accumulator chamber. Both inlet and outlet valves are supposed to be closed during isovolumic contraction and relaxation phases, and so the accumulator should have no influence on the system during these two phases. We believe that this is essential to allow for switching timing. The accumulator should discharge the accumulated blood during the filling phase by opening the outlet valve and keeping the inlet valve closed. It is important that the discharging process is completely finished during this phase such that the accumulator is totally empty before the start of the next cardiac cycle. This cannot be accomplished without the need to an external input, such as a controlled force source, that can manipulate the discharging process (represented by $F$ in Figure 4.1 and $U(t)$ in Figure 4.3). Table 4.1 describes the status of the accumulator and its inlet and outlet valves during each phase of the cardiac cycle. 


\begin{tabular}{|c|c|c|c|c|}
\hline \multirow{2}{*}{\multicolumn{2}{|c|}{ Cardiac cycle phases }} & \multicolumn{2}{|c|}{ Accumulator valves state } & \multirow{3}{*}{$\begin{array}{c}\begin{array}{c}\text { Accumulator } \\
\text { status }\end{array} \\
\text { Empty }\end{array}$} \\
\hline & & Inlet & Outlet & \\
\hline Isovolumic contraction & \multirow{2}{*}{ Systole } & Closed & Closed & \\
\hline Ejection & & Open & Closed & Filling \\
\hline Isovolumic relaxation & \multirow{2}{*}{ Diastole } & Closed & Closed & Filled \\
\hline Filling & & Closed & Open & Discharging \\
\hline
\end{tabular}

Table 4.1. Status of the accumulator and its inlet and outlet valves during each phase of the cardiac cycle.

The selection of the capacitance value of $C_{6}$, which represents the capacitance of the accumulator, was based on the following equation:

$$
C=\frac{Q}{v}
$$

The above equation is the electric circuit version of equation (3.3), where $C$ is capacitance (in Coulomb/Volt, also called Farad), $Q$ is charge (in Coulomb), and $v$ is voltage (in Volts). Thus, the charge $Q$ and voltage $v$ are analogue to volume $V$ and pressure $P$ in equation (3.3); respectively, while the capacitance and compliance are equivalent. We roughly designed the capacitance value of $C_{6}$ such that the maximum value for $Q$ that we can obtain is an above average level of stroke volume $(\sim 80 \mathrm{ml})$, where this occurs when the voltage $v$ across $C_{6}$ is maximum (roughly approximated to be around the maximum left ventricular pressure of $\sim 160 \mathrm{mmHg}$ ). Thus, the value selected for $C_{6}$ was roughly obtained by applying equation (3.3) according to the following:

$$
C_{6}=\frac{80 \mathrm{ml}}{160 \mathrm{mmHg}}=0.5 \mathrm{ml} / \mathrm{mmHg}(\text { or Farad })
$$




\subsection{Methodology}

The cardiovascular system model with accumulator device (Figure 4.3) consists of 6 state variables, with the $6^{\text {th }}$ state variable representing the pressure inside the accumulator chamber (the voltage across the capacitor $C_{6}$ in its electric circuit analogue). The cardiovascular system with the accumulator is described by the following linear, timevarying, state-space system:

$$
\begin{aligned}
& \dot{x}(t)=A(t) x(t)+B(t) u(t) \\
& y(t)=C(t) x(t)+D(t) u(t)
\end{aligned}
$$

where $x(t)$ represents the state vector, $u(t)$ represents the input which is described by the voltage source $U(t)$ in Figure 4.3, and $y(t)$ represents the system's output, which is the flow rate of discharged blood from the accumulator device. $A(t)$, which is a 6x6 matrix, and $B(t)$, which is a $6 \times 1$ vector, represent the system's properties. On the other hand, $C(t)$, a $1 \times 6$ vector, and $D(t)$, a scalar, both describe our chosen output variable. The state variables of the system illustrated in Figure 4.3 are summarized in Table 4.2.

\begin{tabular}{c|l|c}
\hline $\begin{array}{c}\text { State-space } \\
\text { variable }\end{array}$ & \multicolumn{1}{|c}{ Physiological meaning (unit) } & Abbreviation \\
\hline$x_{1}$ & Left ventricular pressure $(\mathrm{mmHg})$ & LVP \\
\hline$x_{2}$ & Left atrial pressure $(\mathrm{mmHg})$ & LAP \\
\hline$x_{3}$ & Arterial pressure $(\mathrm{mmHg})$ & AP \\
\hline$x_{4}$ & Aortic pressure $(\mathrm{mmHg})$ & AoP \\
\hline$x_{5}$ & Aortic flow $(\mathrm{ml} / \mathrm{sec})$ & $\mathrm{Q}_{\mathrm{A}}$ \\
\hline$x_{6}$ & Pressure inside accumulator $(\mathrm{mmHg})$ & $\mathrm{P}_{\mathrm{acc}}$ \\
\hline
\end{tabular}

Table 4.2. State variables used in the cardiovascular system with accumulator. 


\section{Isovolumic contraction (and relaxation) phases}

As described earlier in chapter 3, during the isovolumic contraction and relaxation phases, both the mitral valve and aortic valve are closed (corresponding switches are open circuited). Figure 4.4 illustrates a schematic for the circuit model describing the cardiovascular system with the accumulator device during the isovolumic contraction (and relaxation) phases. A simplified circuit model for the system during these phases is shown in Figure 4.5. Both the inlet and outlet valves of the accumulator are closed (switches are open circuited), and so the implementation of the accumulator device should not have any impact on the system during the isovolumic contraction and relaxation phases.

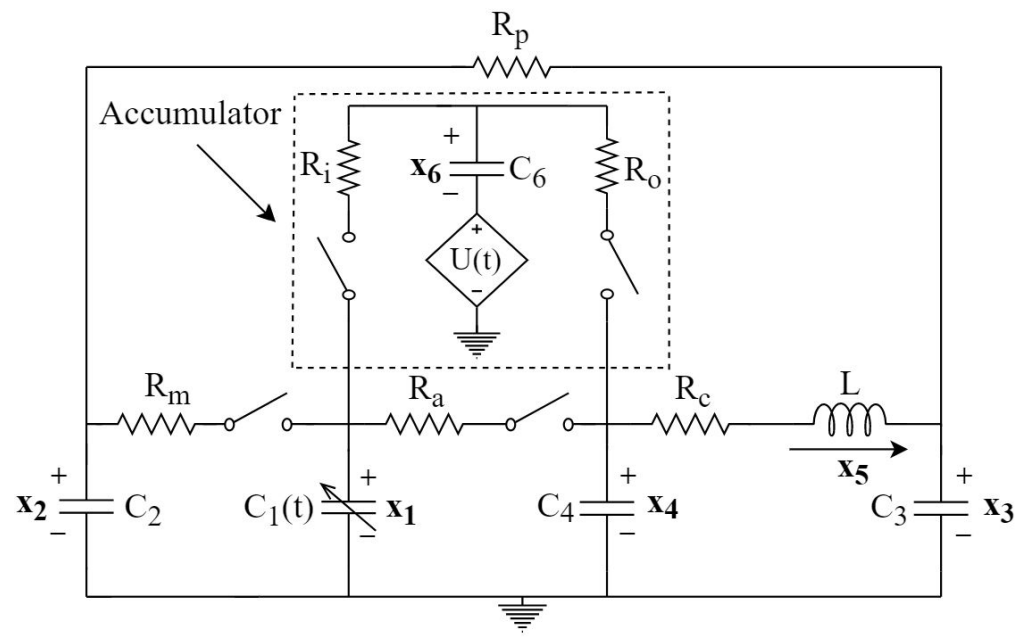

Figure 4.4. Equivalent circuit for the cardiovascular system with the accumulator device during the isovolumic contraction and relaxation phases. Both the mitral and aortic valves are closed (thus both switches are open circuited), and both the accumulator inlet and outlet valves are also closed (switches are open circuited).

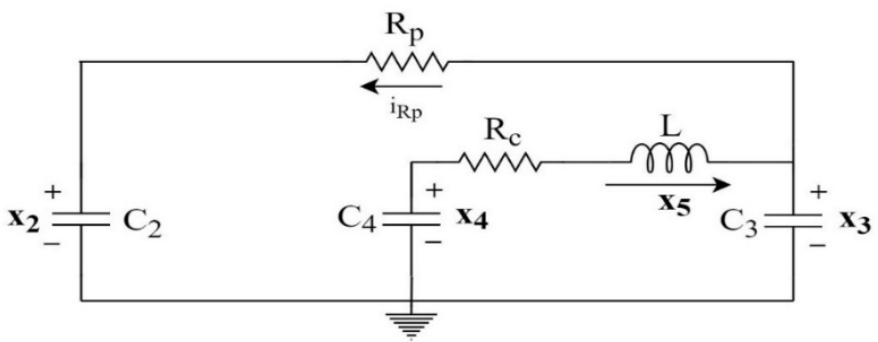

Figure 4.5. Simplified circuit model for the cardiovascular system with accumulator device during isovolumic contraction and relaxation phases. 
The circuit in Figure 4.5 is identical to that of the cardiovascular system with no accumulator (Figure 3.8) which was described in chapter 3. Since the accumulator is not affecting the system, the state equations for the cardiovascular system with the accumulator (Figure 4.3) during the isovolumic contraction and relaxation phases (Figure 4.4) follow the system equation derived in (3.18) with just one difference arising from the addition of the new state variable (pressure inside the accumulator, $x_{6}$ ) which can be described as:

$$
\dot{x}_{6}(t)=0
$$

The above equation was derived from the fact that there should be no change in the volume inside the accumulator due to the closure of both the inlet and outlet valves of the accumulator. Thus, the system equation describing the model in Figure 4.4 can now be written as follows:

$$
\left[\begin{array}{l}
\dot{x}_{1}(t) \\
\dot{x}_{2}(t) \\
\dot{x}_{3}(t) \\
\dot{x}_{4}(t) \\
\dot{x}_{5}(t) \\
\dot{x}_{6}(t)
\end{array}\right]=\left[\begin{array}{cccccc}
\frac{\dot{E}(t)}{E(t)} & 0 & 0 & 0 & 0 & 0 \\
0 & \frac{-1}{R_{p} C_{2}} & \frac{1}{R_{p} C_{2}} & 0 & 0 & 0 \\
0 & \frac{1}{R_{p} C_{3}} & \frac{-1}{R_{p} C_{3}} & 0 & \frac{1}{C_{3}} & 0 \\
0 & 0 & 0 & 0 & \frac{-1}{C_{4}} & 0 \\
0 & 0 & \frac{-1}{L} & \frac{1}{L} & \frac{-R_{c}}{L} & 0 \\
0 & 0 & 0 & 0 & 0 & 0
\end{array}\right] *\left[\begin{array}{l}
x_{1}(t) \\
x_{2}(t) \\
x_{3}(t) \\
x_{4}(t) \\
x_{5}(t) \\
x_{6}(t)
\end{array}\right]
$$




\section{Ejection phase}

The cardiovascular system with the accumulator (Figure 4.3) during the ejection phase is illustrated in Figure 4.6, and its equivalent simplified circuit is presented in Figure 4.7. During this phase, blood is ejected naturally from the left ventricle into the aorta through the aortic valve, which opens during this phase since the pressure inside the left ventricle exceeds that in the aorta. The addition of the accumulator device, with its inlet valve being open during this phase, while its outlet valve is being closed, allows for a new path for the blood that is pumped out of the left ventricle. Thus, the accumulator is filled with blood during this phase, with the accumulation amount being dependent mainly on the value of the inlet resistance $\left(R_{i}\right)$.

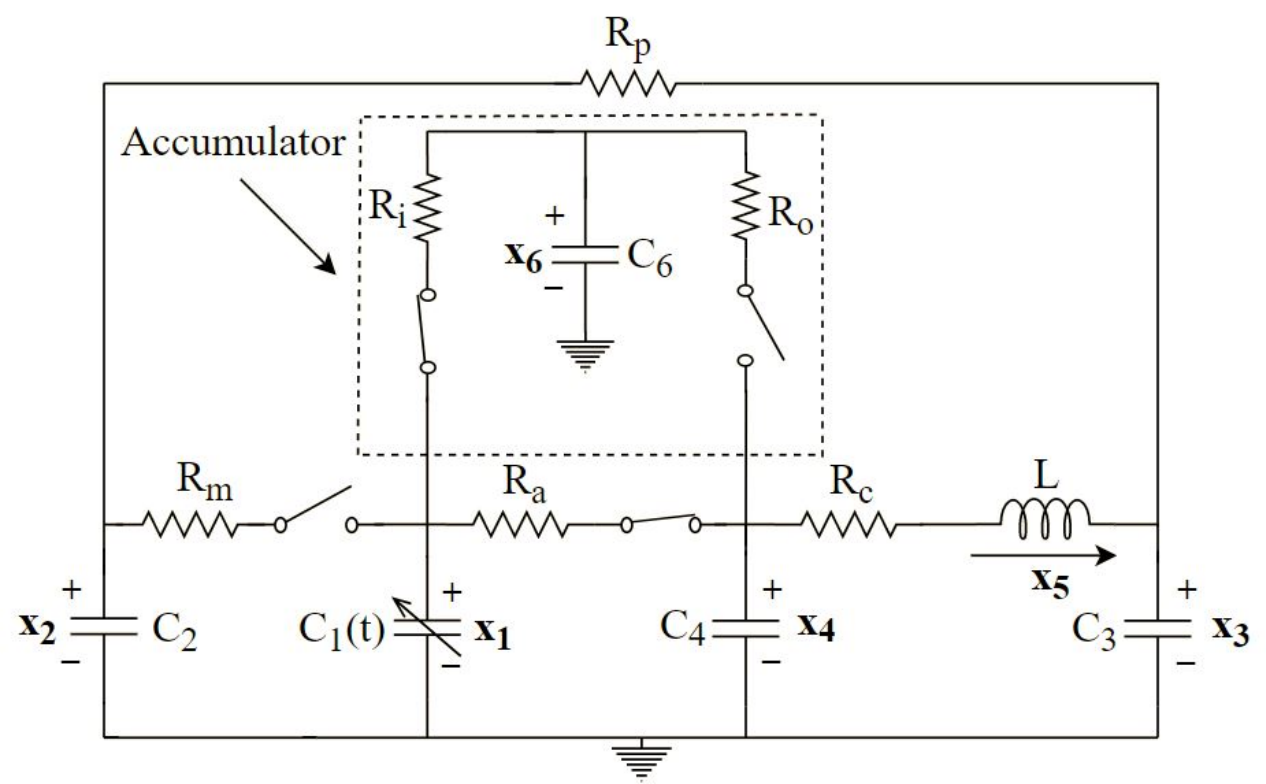

Figure 4.6. Equivalent circuit for the cardiovascular system with the accumulator device during ejection phase. The mitral valve is closed (switch is open circuited), whereas the aortic valve is open (switch is close circuited). The accumulator's inlet valve is open (switch is closed circuit) to allow for blood accumulation, whereas the outlet valve is closed (switch is open circuit). 


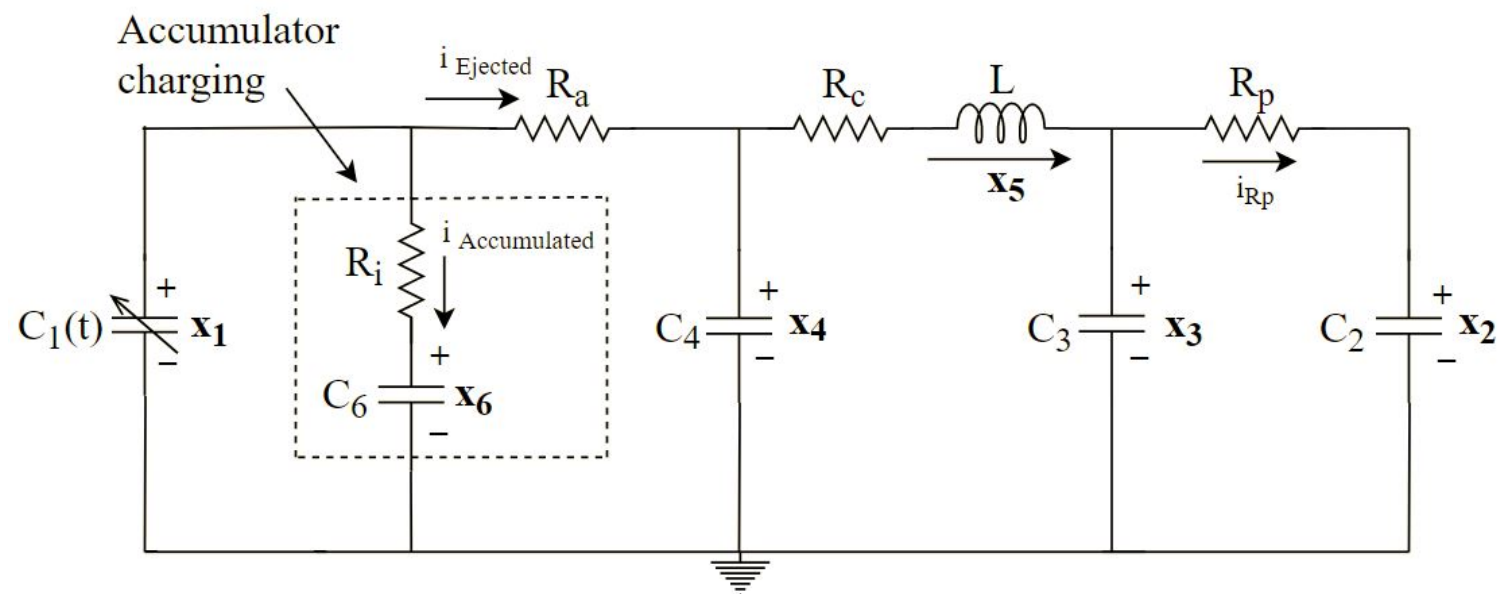

Figure 4.7. Simplified circuit model for the cardiovascular system with the accumulator device during the ejection phase.

Note that the voltage source $U(t)$ (or pressure pump F), which represents the driving force to the accumulator that pushes the accumulator piston downward in order to control and regulate pumping the blood out of the accumulator into the aorta, is not shown in the circuit of Figure 4.6 and Figure 4.7 (represented by applying a 0 volt voltage source, i.e. grounding). The reason for that is because we do not want to apply any force against the accumulation process during this phase such that to make the system less complicated since we can control the accumulation process by regulating the value of $R_{i}$. Thus, for the electric model presented in Figure 4.6, the flow rate of the blood being accumulated will be governed by the first order exponential increase in the pressure inside accumulator, which is dependent on the value of inlet resistance and compliance (capacitance) of the accumulator chamber (time constant $\tau=R_{i} * C_{6}$ ).

To derive the state equations for the dynamics describing the cardiovascular system with the accumulator device during the ejection phase, we will consider the simplified circuit model shown in Figure 4.7. Recalling the elastance function, equation (3.6): 


$$
E(t)=\frac{L V P(t)}{L V V(t)-V_{0}}
$$

and its derivative with respect to time, equation (3.12):

$$
L \dot{V} P(t)=\dot{x}_{1}(t)=\dot{E}(t) *\left(L V V(t)-V_{0}\right)+E(t) * L \dot{V} V(t)
$$

To solve for $\dot{x}_{1}(t)$, we need to find $L \dot{V} V(t)$, the rate of change in the left ventricular volume, during the ejection phase, which can be described as follows:

$$
L \dot{V} V(t)=Q_{\text {in }}-Q_{\text {out }}=0-\left(i_{\text {Ejected }}+i_{\text {accumulated }}\right)
$$

where $i_{E j e c t e d}=\frac{x_{1}(t)-x_{4}(t)}{R_{a}}$, and $i_{\text {accumulated }}=\frac{x_{1}(t)-x_{6}(t)}{R_{i}}$, thus:

$$
L \dot{V} V(t)=-\left(\frac{1}{R_{a}}+\frac{1}{R_{i}}\right) x_{1}(t)+\frac{1}{R_{a}} x_{4}(t)+\frac{1}{R_{i}} x_{6}(t)
$$

Hence the state equation for $x_{1}(t)$ becomes:

$$
\dot{x}_{1}(t)=\left(\frac{\dot{E}(t)}{E(t)}-E(t) *\left(\frac{1}{R_{a}}+\frac{1}{R_{i}}\right)\right) x_{1}(t)+\frac{E(t)}{R_{a}} x_{4}(t)+\frac{E(t)}{R_{i}} x_{6}(t)
$$

The current flowing through $R_{p}$ in the direction shown in Figure 4.7 is:

$$
i_{R_{p}}=C_{2} \dot{x}_{2}(t)=x_{5}(t)-C_{3} \dot{x}_{3}(t)
$$


Applying KVL to the right most loop in Figure 4.7 using the two definitions of $i_{R_{p}}$ in (4.7), we can find the state equations for $x_{2}(t)$ and $x_{3}(t)$ as follows:

$$
\begin{gathered}
\dot{x}_{2}(t)=\frac{-1}{R_{p} C_{2}} x_{2}(t)+\frac{1}{R_{p} C_{2}} x_{3}(t) \\
\dot{x}_{3}(t)=\frac{1}{R_{p} C_{3}} x_{2}(t)-\frac{1}{R_{p} C_{3}} x_{3}(t)+\frac{1}{C_{3}} x_{5}(t)
\end{gathered}
$$

Applying KCL to the node that is common between $R_{a}, R_{c}$, and $C_{4}$ in Figure 4.7, we obtain:

$$
\frac{x_{1}(t)-x_{4}(t)}{R_{a}}=C_{4} \dot{x}_{4}(t)+x_{5}(t)
$$

Re-arranging the above equation, we can solve for $\dot{x}_{4}(t)$ :

$$
\dot{x}_{4}(t)=\frac{1}{R_{a} C_{4}} x_{1}(t)-\frac{1}{R_{a} C_{4}} x_{4}(t)-\frac{1}{C_{4}} x_{5}(t)
$$

Applying KVL on the second loop from the right of Figure 4.7, we obtain:

$$
-x_{4}(t)+R_{c} x_{5}(t)+L * \dot{x}_{5}(t)+x_{3}(t)=0
$$

Solving for $\dot{x}_{5}(t)$, we obtain:

$$
\dot{x}_{5}(t)=\frac{-1}{L} x_{3}(t)+\frac{1}{L} x_{4}(t)-\frac{R_{c}}{L} x_{5}(t)
$$


Finally, applying the equation of the current flowing through a capacitor to solve for $\dot{x}_{6}(t)$, we obtain:

$$
\begin{gathered}
\frac{x_{1}(t)-x_{6}(t)}{R_{i}}=C_{6} x_{6}(t) \\
\dot{x}_{6}(t)=\frac{1}{R_{i} C_{6}} x_{1}(t)-\frac{1}{R_{i} C_{6}} x_{6}(t)
\end{gathered}
$$

Putting equations (4.6), (4.8), (4.9), (4.10), (4.11), and (4.12) together to solve for the matrix $A(t)$, where $\dot{x}(t)=A(t) * x(t)$, we obtain:

$$
\left[\begin{array}{c}
\dot{x}_{1}(t) \\
\dot{x}_{2}(t) \\
\dot{x}_{3}(t) \\
\dot{x}_{4}(t) \\
\dot{x}_{5}(t) \\
\dot{x}_{6}(t)
\end{array}\right]=\left[\begin{array}{cccccc}
\frac{\dot{E}(t)}{E(t)}-E(t)\left(\frac{1}{R_{a}}+\frac{1}{R_{i}}\right) & 0 & 0 & \frac{E(t)}{R_{a}} & 0 & \frac{E(t)}{R_{i}} \\
0 & \frac{-1}{R_{p} C_{2}} & \frac{1}{R_{p} C_{2}} & 0 & 0 & 0 \\
0 & \frac{1}{R_{p} C_{3}} & \frac{-1}{R_{p} C_{3}} & 0 & \frac{1}{C_{3}} & 0 \\
\frac{1}{R_{a} C_{4}} & 0 & 0 & \frac{-1}{R_{a} C_{4}} & \frac{-1}{C_{4}} & 0 \\
0 & 0 & \frac{-1}{L} & \frac{1}{L} & \frac{-R_{c}}{L} & 0 \\
\frac{1}{R_{i} C_{6}} & 0 & 0 & 0 & 0 & \frac{-1}{R_{i} C_{6}}
\end{array}\right] *\left[\begin{array}{l}
x_{1}(t) \\
x_{2}(t) \\
x_{3}(t) \\
x_{4}(t) \\
x_{5}(t) \\
x_{6}(t)
\end{array}\right]
$$




\section{Filling phase}

By the end of the ejection phase, the accumulator is filled with blood, with a quantity that depends on different factors where one of the most importance factors (that our study will focus on) is the accumulator's inlet resistance $R_{i}$. During the isovolumetric relaxation phase, which immediately follows the ejection phase, both accumulator valves are closed, which means there is no change in the volume of accumulated blood. The process of discharging the accumulated blood back to the aorta starts at the onset of the cardiac cycle's filling phase (when the mitral valve opens while the aortic valve is closed). The discharging process requires an external force in order to force the pumping out of the blood from the low-pressure side of the accumulator into the high-pressure side of the aorta. This external force is represented by the varying voltage source $U(t)$ as shown in the circuit of Figure 4.3. The external force will be controlled such that the blood discharging rate follows an optimized flow rate trajectory that ensures emptying the accumulator during this phase with the least disturbance possible in the resulting waveform profile of the aortic pressure.

Figure 4.8 illustrates the circuit describing the cardiovascular system with the accumulator device during the filling phase of the cardiac cycle. The equivalent simplified circuit is shown in Figure 4.9, where the voltage source $U(t)$ represents the input to the system. Figure 4.10 shows another version for the simplified circuit model where the accumulator is represented by a controlled current source instead of a varying voltage source in series with a capacitor as in Figure 4.9. 


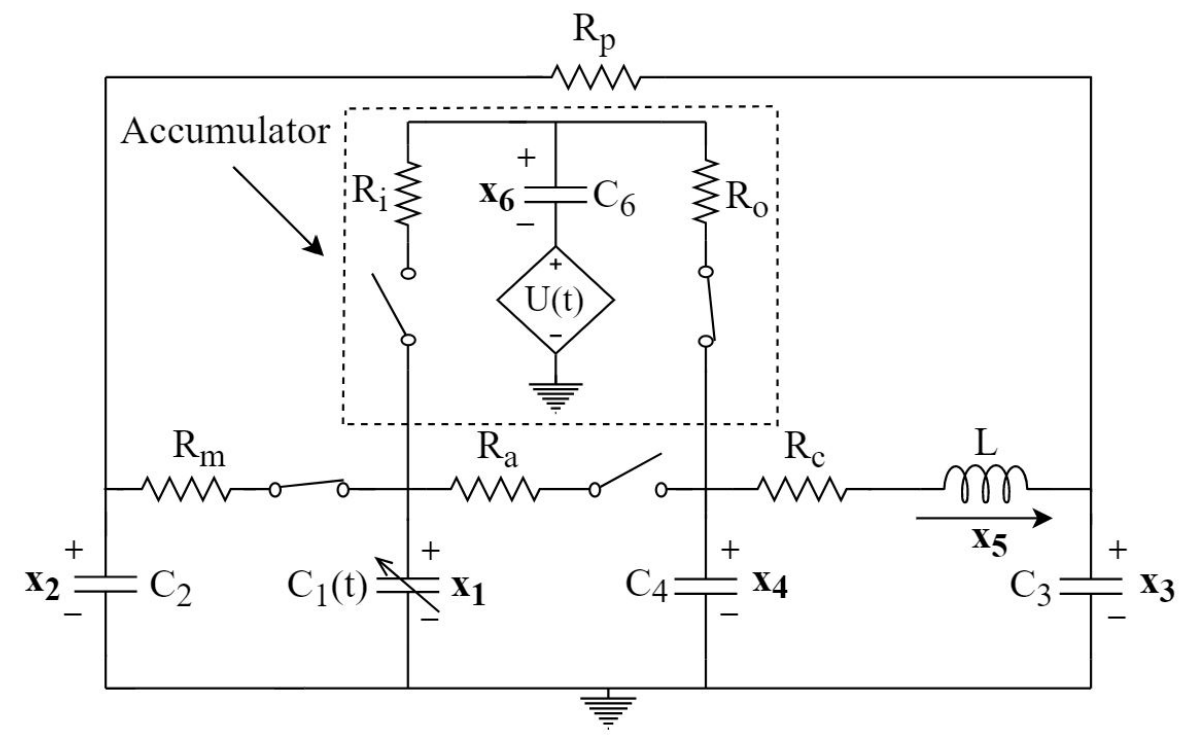

Figure 4.8. Equivalent circuit for the cardiovascular system with the accumulator device during the filling phase. The mitral valve is open (thus switch is close circuited), whereas the aortic valve is closed (thus switch is open circuited). The accumulator inlet valve is closed (switch is open circuit), whereas the accumulator's outlet valve is open (switch is closed circuit) to allow for blood to discharge back to the aorta.

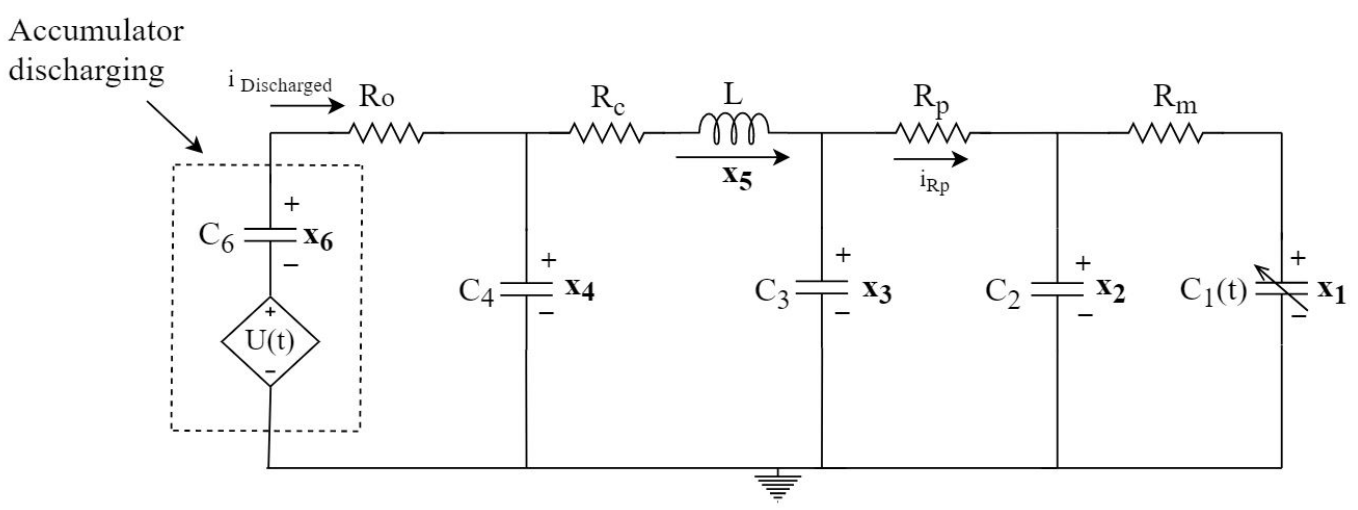

Figure 4.9. Simplified circuit model for the cardiovascular system with the accumulator device during the filling phase. 


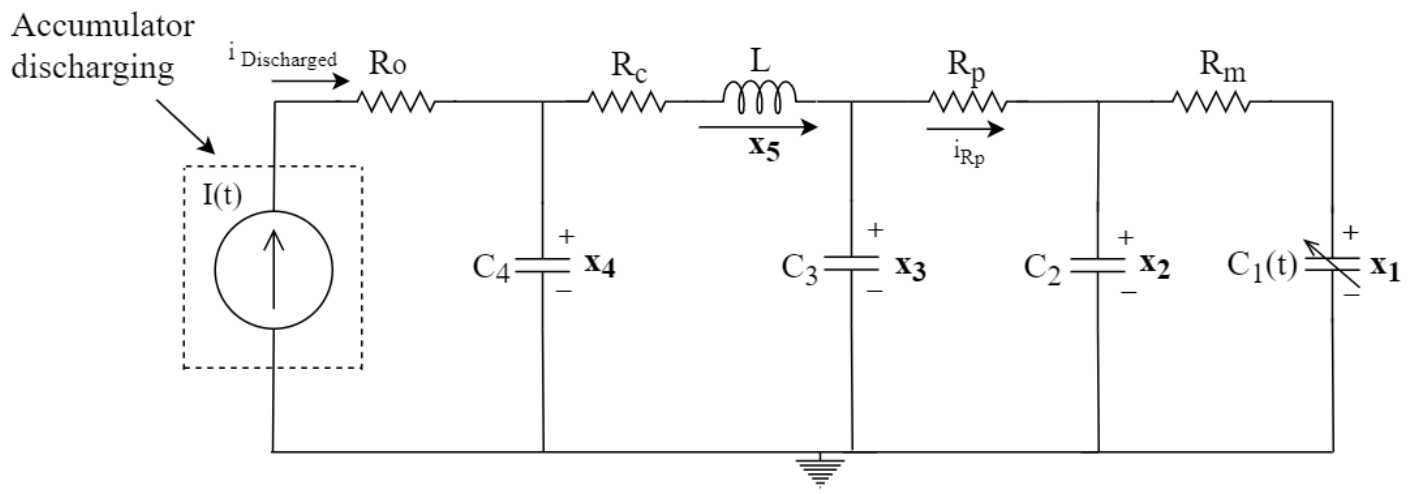

Figure 4.10. Simplified circuit model for the cardiovascular system with the accumulator device during the filling phase. We represent the accumulator here as a controlled current source instead of a varying voltage source in series with a capacitor that we aim to discharge.

We now derive the system equations for the circuit illustrated in Figure 4.9. Let us recall the derivative of the elastance function in equation (3.12):

$$
\dot{x}_{1}(t)=\dot{E}(t) *\left(L V V(t)-V_{0}\right)+E(t) * L \dot{V} V(t)
$$

where $L \dot{V} V(t)$ representing the rate of change in the left ventricular volume during the filling phase according to the following:

$$
L \dot{V} V(t)=Q_{\text {in }}-Q_{\text {out }}=\frac{x_{2}(t)-x_{1}(t)}{R_{m}}-0=\frac{x_{2}(t)-x_{1}(t)}{R_{m}}
$$

Thus, the equation for $\dot{x}_{1}(t)$ becomes:

$$
\dot{x}_{1}(t)=\left(\frac{\dot{E}(t)}{E(t)}-\frac{E(t)}{R_{m}}\right) x_{1}(t)+\frac{E(t)}{R_{m}} x_{2}(t)
$$

The current flowing through $R_{p}$ in the direction shown in Figure 4.9 can be described as:

$$
i_{R_{p}}=C_{2} \dot{x}_{2}(t)+\frac{x_{2}(t)-x_{1}(t)}{R_{m}}=x_{5}(t)-C_{3} \dot{x}_{3}(t)
$$


Applying KVL to the second loop from the right side of the circuit shown in Figure 4.9 using the two definitions of $i_{R_{p}}$ in equation (4.15), we obtain:

$$
\begin{gathered}
x_{2}(t)-x_{3}(t)+R_{p} *\left(C_{2} \dot{x}_{2}(t)+\frac{x_{2}(t)-x_{1}(t)}{R_{m}}\right)=0 \\
x_{2}(t)-x_{3}(t)+R_{p} *\left(x_{5}(t)-C_{3} \dot{x}_{3}(t)\right)=0
\end{gathered}
$$

Re-arranging the above equations, we obtain:

$$
\begin{gathered}
\dot{x}_{2}(t)=\frac{1}{R_{m} C_{2}} x_{1}(t)-\frac{1}{C_{2}} *\left(\frac{1}{R_{p}}+\frac{1}{R_{m}}\right) x_{2}(t)+\frac{1}{R_{p} C_{2}} x_{3}(t) \\
\dot{x}_{3}(t)=\frac{1}{R_{p} C_{3}} x_{2}(t)-\frac{1}{R_{p} C_{3}} x_{3}(t)+\frac{1}{C_{3}} x_{5}(t)
\end{gathered}
$$

Applying KCL to the node that is common between $R_{o}, R_{c}$, and $C_{4}$ in Figure 4.9, we obtain:

$$
\frac{x_{6}(t)+U(t)-x_{4}(t)}{R_{o}}=C_{4} \dot{x}_{4}(t)+x_{5}(t)
$$

Re-arranging the previous equation to solve for $\dot{x}_{4}(t)$, we obtain:

$$
\dot{x}_{4}(t)=\frac{-1}{R_{o} C_{4}} x_{4}(t)-\frac{1}{C_{4}} x_{5}(t)+\frac{1}{R_{o} C_{4}} x_{6}(t)+\frac{1}{R_{o} C_{4}} U(t)
$$


Applying KVL to the second loop from the left side of the circuit shown in Figure 4.9, we obtain:

$$
-x_{4}(t)+R_{c} x_{5}(t)+L * \dot{x}_{5}(t)+x_{3}(t)=0
$$

which can be re-arranged according to the following:

$$
\dot{x}_{5}(t)=\frac{-1}{L} x_{3}(t)+\frac{1}{L} x_{4}(t)-\frac{R_{c}}{L} x_{5}(t)
$$

To derive the equation for the last state, $\dot{x}_{6}(t)$, we simply apply the equation describing the current flowing through a capacitor, equation (3.9), as well as $\mathrm{Ohm}$ 's law, to obtain:

$$
C_{6} * \frac{d}{d t}\left(-x_{6}(t)\right)=\frac{U(t)+x_{6}(t)-x_{4}(t)}{R_{o}}
$$

which can be re-arranged according to the following:

$$
\dot{x}_{6}(t)=\frac{1}{R_{o} C_{6}} x_{4}(t)-\frac{1}{R_{o} C_{6}} x_{6}(t)-\frac{1}{R_{o} C_{6}} U(t)
$$

The output, $y(t)$, is represented by the current (blood) that is discharged by the accumulator as follows:

$$
y(t)=\frac{U(t)+x_{6}(t)-x_{4}(t)}{R_{o}}=\frac{1}{R_{o} C_{6}} x_{4}(t)-\frac{1}{R_{o} C_{6}} x_{6}(t)-\frac{1}{R_{o} C_{6}} U(t)
$$


Putting equations (4.14), (4.16), (4.17), (4.18), (4.19), (4.20), and (4.21) together, we can now describe the cardiovascular system with the accumulator device during filling phase (Figure 4.8) according to the following system equations:

$$
\dot{\boldsymbol{x}}(\boldsymbol{t})=\left[\begin{array}{cccccc}
\frac{\dot{E}(t)}{E(t)}-\frac{E(t)}{R_{m}} & \frac{E(t)}{R_{m}} & 0 & 0 & 0 & 0 \\
\frac{1}{R_{m} C_{2}} & \frac{-1}{C_{2}} *\left(\frac{1}{R_{p}}+\frac{1}{R_{m}}\right) & \frac{1}{R_{p} C_{2}} & 0 & 0 & 0 \\
0 & \frac{1}{R_{p} C_{3}} & \frac{-1}{R_{p} C_{3}} & 0 & \frac{1}{C_{3}} & 0 \\
0 & 0 & 0 & \frac{-1}{R_{o} C_{4}} & \frac{-1}{C_{4}} & \frac{1}{R_{o} C_{4}} \\
0 & 0 & \frac{-1}{L} & \frac{1}{L} & \frac{-R_{c}}{L} & 0 \\
\boldsymbol{y}(\boldsymbol{t}) & =\left[\begin{array}{cccccc}
0 & 0 & 0 & \frac{1}{R_{o}} & 0 & \frac{-1}{R_{o}}
\end{array}\right] \boldsymbol{x}(\boldsymbol{t})+\left[\frac{1}{R_{o}}\right] \boldsymbol{u}(\boldsymbol{t}) & \frac{1}{R_{o} C_{6}} & 0 & \frac{-1}{R_{o} C_{6}}
\end{array}\right]+\left[\begin{array}{c}
0 \\
0 \\
0 \\
1 \\
\frac{R_{o} C_{4}}{0} \\
\frac{-1}{R_{o} C_{6}}
\end{array}\right] \boldsymbol{u}(\boldsymbol{t})
$$

The system in (4.22) requires a controller that can manipulate the control input $U(t)$ in order to achieve a desired output $y(t)$, which represents the actual flow rate of the blood discharged from the accumulator device back to the arterial system. The controller design will be the subject of the next chapter, and so in this section, we will assume that the actual flow rate is already known without investigating any control mechanism to achieve it. Thus, we refer to the circuit in Figure 4.10, where we considered our input as a controlled current source with a pre-determined desired signal of current (discharged blood flow rate). This is going to be a signal of finite flow rate that is determined by two key factors: the total amount of blood that was accumulated during the ejection phase that needs to be redelivered back to the body, and the time interval, i.e. $t_{0}$ and $t_{f}$, of the discharging process which occurs during the filling phase of the cardiac cycle. The area under the curve of this 
signal of flow rate should be equal to the total volume of accumulated blood to be discharged.

The system in Figure 4.10, which considers the current source as the driving force of the system, can be described according to the following system equation:

$$
\left[\begin{array}{l}
\dot{x}_{1}(t) \\
\dot{x}_{2}(t) \\
\dot{x}_{3}(t) \\
\dot{x}_{4}(t) \\
\dot{x}_{5}(t)
\end{array}\right]=\left[\begin{array}{ccccc}
\frac{\dot{E}(t)}{E(t)}-\frac{E(t)}{R_{m}} & \frac{E(t)}{R_{m}} & 0 & 0 & 0 \\
\frac{1}{R_{m} C_{2}} & \frac{-1}{C_{2}} *\left(\frac{1}{R_{p}}+\frac{1}{R_{m}}\right) & \frac{1}{R_{p} C_{2}} & 0 & 0 \\
0 & \frac{1}{R_{p} C_{3}} & \frac{-1}{R_{p} C_{3}} & 0 & \frac{1}{C_{3}} \\
0 & 0 & 0 & 0 & \frac{-1}{C_{4}} \\
0 & 0 & \frac{-1}{L} & \frac{1}{L} & \frac{-R_{c}}{L}
\end{array}\right] *\left[\begin{array}{c}
x_{1}(t) \\
x_{2}(t) \\
x_{3}(t) \\
x_{4}(t) \\
x_{5}(t)
\end{array}\right]+\left[\begin{array}{c}
0 \\
0 \\
0 \\
\frac{1}{C_{4}} \\
0
\end{array}\right] \boldsymbol{I}(\boldsymbol{t})
$$

where $I(t)$ in the above system represents the desired current (discharged blood flow rate) signal in the system in Figure 4.10, which was also labeled as $i_{\text {Discharged }}$. Note that the only differences between the system equations in (4.23), which describe the system in Figure 4.10, and the system equations in (4.22), which describe the system in Figure 4.9, are the absence of the sixth state which represents the voltage across $C_{6}$ (the pressure inside the accumulator in its mechanical analogue) and the equation describing $\dot{x}_{4}(t)$, where $x_{4}(t)$ represents the voltage across $C_{4}$ (aortic pressure). Note that the absence of the sixth state $x_{6}(t)$ in the system described in (4.23) is equivalent to showing it with an additional row and column of zeros in the $A(t)$ matrix and a sixth element of zero in the $B$ vector. To derive the equation for $\dot{x}_{4}(t), \mathrm{KCL}$ was applied to the node joining $R_{o}, R_{c}$, and $C_{4}$ according to the following:

$$
I(t)=i_{\text {Discharged }}=C_{4} \dot{x}_{4}(t)+x_{5}(t)
$$


Re-arranging the previous equation, we obtain:

$$
\dot{x}_{4}(t)=\frac{-1}{C_{4}} x_{5}(t)+\frac{1}{C_{4}} I(t)
$$

\subsection{Simulation Results}

We first test our hypothesis of the novel idea that implementing the accumulator device into the cardiovascular system, as illustrated in Figure 4.1 and Figure 4.3, could help in improving blood pressure measurements. This can be illustrated by showing how accumulating a partial amount of the blood being pumped out of the left ventricle during the ejection phase, while assuring the re-delivery of this accumulated blood back to the arterial system during the filling phase (i.e. before the end of the cardiac cycle), can cause some hemodynamical changes. We will investigate the relationship between the amount of blood accumulated, which is mainly governed by the resistance of the accumulator inlet $\left(R_{i}\right)$, and the corresponding effect on pressure and end-diastolic volume. We will also investigate the influence of implementing the accumulator idea on modulating the time duration of cardiac cycle phases. We finally investigate the effect of the waveform shape of flow rate for the blood being discharged by the accumulator on the corresponding waveform profile of the aortic pressure. This is done by applying different flow rate waveform shapes for the discharged blood, such as the sinusoidal and step functions as well as additional functions.

The simulation work was implemented in MATLAB using numerical computations to solve the state-space equations describing the cardiovascular system with the 
accumulator device of Figure 4.3. The following parametric values were used: heart rate 75 beats $/$ min (corresponding to cardiac cycle period of $0.8 \mathrm{sec}$ ), $E_{\max }=2.0 \mathrm{mmHg} / \mathrm{ml}$, $E_{\min }=0.05 \mathrm{mmHg} / \mathrm{ml}$, and $V_{0}=10 \mathrm{ml}$. The other parametric values followed those shown earlier in Table 3.3.

In this section, we analyze the cardiovascular system model with the accumulator device in Figure 4.3 which was described by different system equations, depending on the phase of the cardiac cycle. We saw that the equation in (4.4) described the system behavior during the isovolumic contraction and relaxation phases, the equation in (4.13) described the system behavior during the ejection phase, and the equation in (4.22) described the system behavior during the filling phase.

As a reminder, the controller design will be discussed in the next chapter, and so in this section, we will assume that the signal of actual flow rate of the blood being discharged by the accumulator is known. This will be a signal that is generated theoretically based on using closed-form mathematical functions such that the resulting flow rate signal reflects the desired period of discharging duration, with a discharged amount of blood that is exactly equal to the amount accumulated during the ejection phase. The system equation in (4.22) which describes the dynamical behavior during filling phase, during which the control input $U(t)$ controls the actual flow rate of discharged blood from the accumulator, will not be used now and instead we describe the filling phase by the equivalent system equation in (4.23), whose input is the current source $I(t)$ that we will assume for it to be a known signal of flow rate.

We start our analysis by inducing an unhealthy condition, which will be considered the baseline for our analysis. The unhealthy condition is induced from the same case that 
was introduced earlier (Figure 3.15) but with an increased cardiac output that reflects the normal range for cardiac output. The increase in cardiac output was achieved by changing the simulation initial conditions such that the end-diastolic volume is increased. This step was followed to emulate the action of the body's regulatory mechanism to increase the cardiac output by allowing for more blood flow to the heart (i.e. increasing the end-diastolic volume), also known as venous return $[58,62]$. The last piece of information relates to what is called the Frank-Starling law of the heart, which states that when increased quantities of blood flow into the heart, this stretches the heart muscle more causing more force of contraction which consequently, increases the amount of ejected blood [62]. The resulting hemodynamics for our baseline condition of induced unhealthy case are: systolic and diastolic pressures of 167.8 and $105.4 \mathrm{mmHg}$ respectively, mean arterial pressure of $138.1 \mathrm{mmHg}$, stroke volume of $62.7 \mathrm{ml} /$ beat, cardiac output of $4.7 \mathrm{l} / \mathrm{min}$, and enddiastolic volume of $155.3 \mathrm{ml}$. The corresponding waveforms for LVP, LAP, AoP, LVV, $\mathrm{Q}_{\mathrm{A}}$, and PV-loop are presented in Figure 4.11.
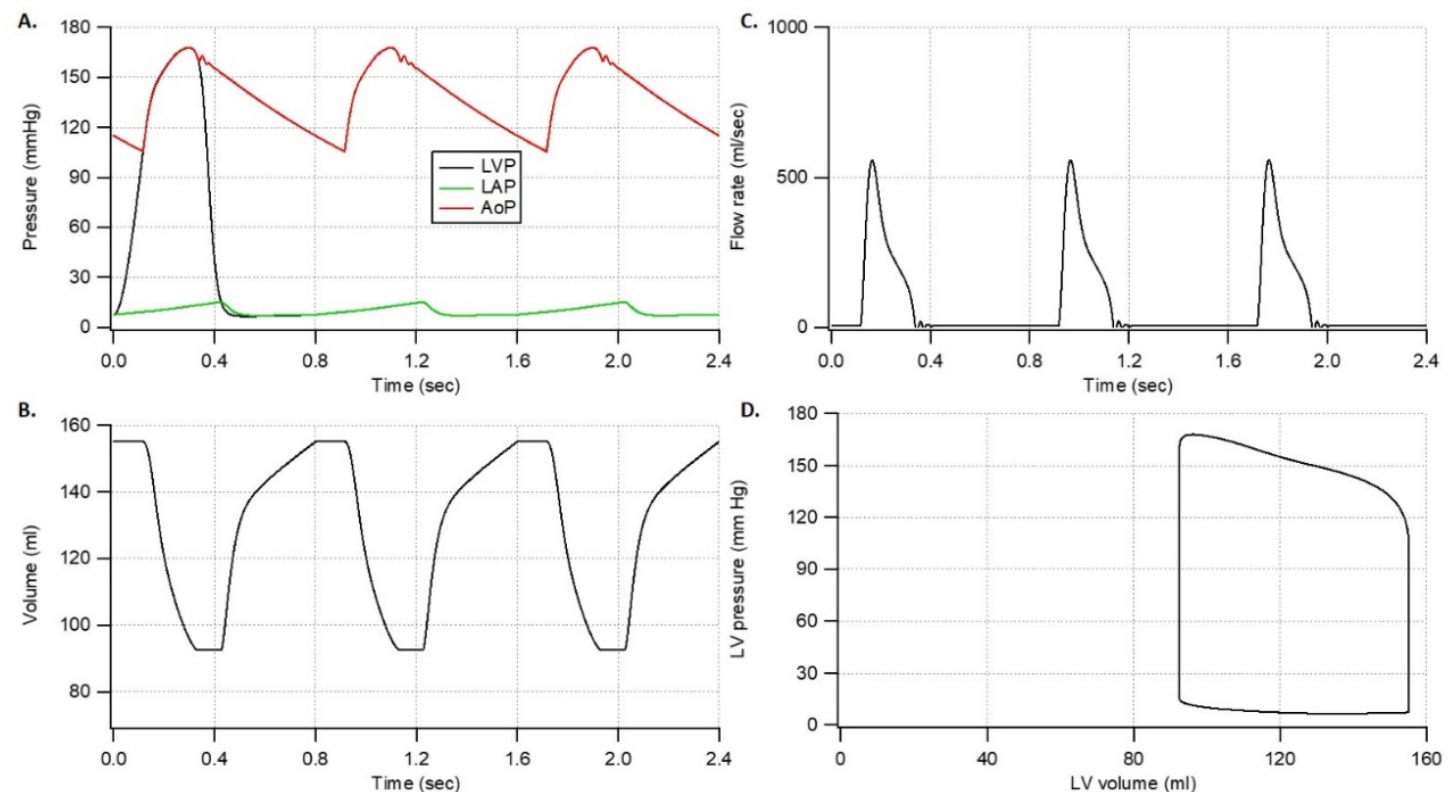

Figure 4.11. Hemodynamics waveforms of our baseline unhealthy condition. 


\section{Testing the Hypothesis That the Accumulator Device Improves Hypertension}

To investigate the implementation of the accumulator device to treat the unhealthy case of the hemodynamics waveforms shown in Figure 4.11, we simulated our system in MATLAB using different values for the accumulator inlet resistance $R_{i}$. Table 4.3 summarizes the results of trying different values for $R_{i}$. Note that as $R_{i}$ decreases, blood accumulation increases, whereas the ejected blood through aortic valve decreases. This caused a reduction in the systolic pressure and an increase in the diastolic pressure, while the MAP was minimally decreased as $R_{i}$ decreased. The first line in Table 4.3 corresponds to the unhealthy condition that we want to treat, which represents our baseline. The inlet resistance value of $0.5 \Omega$ resulted in the lowest systolic pressure and highest diastolic pressure in the data shown in the table, comparing a pressure of $167.8 / 105.4 \mathrm{mmHg}$ in the baseline to $144.1 / 123.2 \mathrm{mmHg}$, in the case of implementing the accumulator device with $R_{i}=0.5 \Omega$. Regardless of the increase in diastolic pressure, which is logical as we will see later, there is a significant reduction in the systolic pressure of $23.7 \mathrm{mmHg}$ (a decrease of $\sim 14 \%)$. When the inlet resistance was decreased more $(<0.5 \Omega)$, i.e. allowing for more accumulation and lesser ejection through aortic valve, the situation became worse compared to the case when $R_{i}=0.5 \Omega$. Both end-diastolic volume (EDV) and cardiac output (CO) decreased as $R_{i}$ decreased, with the decrease in $\mathrm{CO}$ being minimal compared to the decrease in EDV. 


\begin{tabular}{|c|c|c|c|c|c|c|c|c|}
\hline $\begin{array}{c}\text { Accumulator } \\
\text { resistance } \\
\boldsymbol{R}_{\boldsymbol{i}} \\
(O h m s)\end{array}$ & $\begin{array}{c}\text { Ps } \\
\mathrm{mmHg}\end{array}$ & $\begin{array}{c}\text { Pd } \\
\mathrm{mmHg}\end{array}$ & $\begin{array}{c}\text { MAP } \\
\mathrm{mmHg}\end{array}$ & $\begin{array}{c}\mathbf{C O} \\
\mathrm{L} / \mathrm{min}\end{array}$ & $\begin{array}{c}\mathbf{S V} \\
\mathrm{ml} / \text { beat }\end{array}$ & $\begin{array}{c}\text { Ejected } \\
\text { blood } \\
\text { ml/beat }\end{array}$ & $\begin{array}{c}\text { Accumulated } \\
\text { blood } \\
\mathrm{ml} / \text { beat }\end{array}$ & $\begin{array}{c}\text { EDV } \\
\mathrm{ml}\end{array}$ \\
\hline $\begin{array}{c}\text { without } \\
\text { accumulator }\end{array}$ & 167.8 & 105.4 & 138.1 & 4.7 & 62.7 & NA & NA & 155.3 \\
\hline 1000 & 167.8 & 105.5 & 138.1 & 4.7 & 62.7 & 62.7 & 0 & 155.3 \\
\hline 100 & 167.6 & 105.6 & 138.1 & 4.7 & 62.7 & 62.4 & 0.3 & 155.1 \\
\hline 10 & 165.7 & 107.1 & 138 & 4.7 & 62.7 & 59.46 & 3.24 & 154 \\
\hline 5 & 163.7 & 108.7 & 137.9 & 4.7 & 62.66 & 56.44 & 6.22 & 152.7 \\
\hline 2 & 158.6 & 112.6 & 137.6 & 4.69 & 62.57 & 48.66 & 13.9 & 149.7 \\
\hline 1 & 152.3 & 117.3 & 137 & 4.68 & 62.39 & 38.79 & 23.6 & 145.9 \\
\hline 0.75 & 149 & 119.7 & 136.7 & 4.67 & 62.27 & 33.73 & 28.5 & 144 \\
\hline 0.65 & 147.4 & 120.9 & 136.5 & 4.66 & 62.2 & 31.1 & 31.1 & 143 \\
\hline 0.5 & 144.1 & 123.2 & 136.1 & 4.65 & 62.05 & 25.96 & 36.1 & 141.1 \\
\hline 0.25 & 151.1 & 119.8 & 135.2 & 4.63 & 61.71 & 12.49 & 49.2 & 136.1 \\
\hline 0.15 & 156.1 & 114.9 & 134.8 & 4.62 & 61.55 & 4.78 & 56.8 & 133.1 \\
\hline
\end{tabular}

Table 4.3. Hemodynamics of different cases resulting from the implementation of accumulator device with different values for inlet resistance $R_{i}$. Ps, Pd, and MAP stand for systolic, diastolic, and mean arterial pressures respectively. $\mathrm{CO}$ and SV stand for cardiac output and stroke volume. Ejected blood refers to the amount of blood ejected through the aortic valve during the ejection phase, whereas accumulated blood refers to the amount of blood accumulated by the accumulator during the ejection phase and discharged back to the aorta during the filling phase. EDV stands for end-diastolic volume. These simulations were based on heart rate of 75 beats/min.

In another experiment, we investigated the case when the cardiac output was kept unchanged, i.e. keeping the $\mathrm{CO}$ in each case to be equal to that of the baseline condition to avoid the slight decrease in $\mathrm{CO}$ as $R_{i}$ decreases, as we noticed in Table 4.3. This was achieved by changing the initial conditions for simulating each case of $R_{i}$ (the initial conditions used to simulate the cases in Table 4.3 were all identical). Table 4.4 summarizes the results when the $\mathrm{CO}$ was kept unchanged, i.e. $4.7 \mathrm{~L} / \mathrm{min}$ in all the cases. This action 
minimally increased all pressure readings and EDV compared to those in Table 4.3, while the value of $R_{i}=0.5 \Omega$ still resulting in the lowest systolic pressure of $145.6 \mathrm{mmHg}$ (when the $\mathrm{CO}$ is $4.7 \mathrm{~L} / \mathrm{min}$ ), compared to $144.1 \mathrm{mmHg}$ (when the $\mathrm{CO}$ is $4.65 \mathrm{~L} / \mathrm{min}$ as in Table 4.3).

\begin{tabular}{|c|c|c|c|c|c|c|c|c|}
\hline $\begin{array}{c}\text { Accumulator } \\
\text { resistance } \\
\boldsymbol{R}_{\boldsymbol{i}} \\
(\text { Ohms })\end{array}$ & $\begin{array}{c}\mathbf{P s} \\
\mathrm{mmHg}\end{array}$ & $\begin{array}{c}\mathbf{P d} \\
\mathrm{mmHg}\end{array}$ & $\begin{array}{c}\text { MAP } \\
\mathrm{mmHg}\end{array}$ & $\begin{array}{c}\mathbf{C O} \\
\mathrm{L} / \mathrm{min}\end{array}$ & $\begin{array}{c}\mathbf{S V} \\
\mathrm{ml} / \text { beat }\end{array}$ & $\begin{array}{c}\text { Ejected } \\
\text { blood } \\
\mathrm{ml} / \mathrm{beat}\end{array}$ & $\begin{array}{c}\text { Accumulated } \\
\text { blood } \\
\mathrm{ml} / \mathrm{beat}\end{array}$ & $\begin{array}{c}\mathbf{E D V} \\
\mathrm{ml}\end{array}$ \\
\hline $\begin{array}{c}\text { without } \\
\text { accumulator }\end{array}$ & 167.8 & 105.4 & 138.1 & 4.7 & 62.7 & $\mathrm{NA}$ & $\mathrm{NA}$ & 155.3 \\
\hline $\mathbf{1 0 0 0}$ & 167.8 & 105.5 & 138.1 & 4.7 & 62.7 & 62.7 & 0 & 155.3 \\
\hline $\mathbf{1 0 0}$ & 167.6 & 105.6 & 138.1 & 4.7 & 62.7 & 62.4 & 0.3 & 155.1 \\
\hline $\mathbf{1 0}$ & 165.7 & 107.1 & 138 & 4.7 & 62.7 & 59.46 & 3.24 & 154 \\
\hline $\mathbf{5}$ & 163.8 & 108.8 & 138 & 4.7 & 62.72 & 56.49 & 6.23 & 152.9 \\
\hline $\mathbf{2}$ & 159 & 112.9 & 137.9 & 4.7 & 62.72 & 48.77 & 13.9 & 150 \\
\hline $\mathbf{1}$ & 153.1 & 118 & 138 & 4.7 & 62.72 & 39 & 23.7 & 146.6 \\
\hline $\mathbf{0 . 7 5}$ & 150 & 120.6 & 137.7 & 4.7 & 62.72 & 33.97 & 28.7 & 145 \\
\hline $\mathbf{0 . 6 5}$ & 148.6 & 121.9 & 137.7 & 4.7 & 62.7 & 31.4 & 31.4 & 144 \\
\hline $\mathbf{0 . 5}$ & 145.6 & 124.5 & 137.6 & 4.7 & 62.72 & 26.24 & 36.5 & 142.5 \\
\hline $\mathbf{0 . 2 5}$ & 153.5 & 121.8 & 137.4 & 4.7 & 62.72 & 12.69 & 50 & 138.1 \\
\hline $\mathbf{0 . 1 5}$ & 159 & 117.1 & 137.3 & 4.7 & 62.72 & 4.87 & 57.9 & 135.4 \\
\hline
\end{tabular}

Table 4.4. Hemodynamics for different cases resulting from the implementation of the accumulator device with different values of inlet resistance $R_{i}$, while keeping similar cardiac output. 
Figure 4.12 demonstrates the hemodynamical changes caused by implementing the accumulator device. The figure compares the hemodynamics of the unhealthy case with the case of implementing the accumulator device with an inlet resistance value $\left(R_{i}\right)$ of $1 \Omega$. The upper panel shows a decrease in systolic pressure from 167.8 to $152.3 \mathrm{mmHg}$, while the diastolic pressure increased from 105.4 to $117.3 \mathrm{mmHg}$. EDV, represented by the upper flat lines of the waveforms in the middle panel of Figure 4.12, decreased from 155.3 (unhealthy case) to $145.9 \mathrm{ml}$ (treated case). The lower panel describes how blood is delivered to the arterial system during the cardiac cycle. The dashed line represents the flow rate profile of the $62.7 \mathrm{ml} /$ beat $\mathrm{SV}$ of the unhealthy condition which was completely delivered during the ejection phase through the aortic valve, whereas the solid lines show how the flow of a similar amount of stroke volume was split. The solid red line corresponds to the $38.79 \mathrm{ml}$ of blood that was ejected through the aortic valve during the ejection phase, while the solid blue line represents an accumulation of $23.6 \mathrm{ml}$ during the ejection phase that was re-delivered by the accumulator back to the arterial system during the filling phase of the cardiac cycle, creating a total of $62.39 \mathrm{ml} \mathrm{SV}$. 

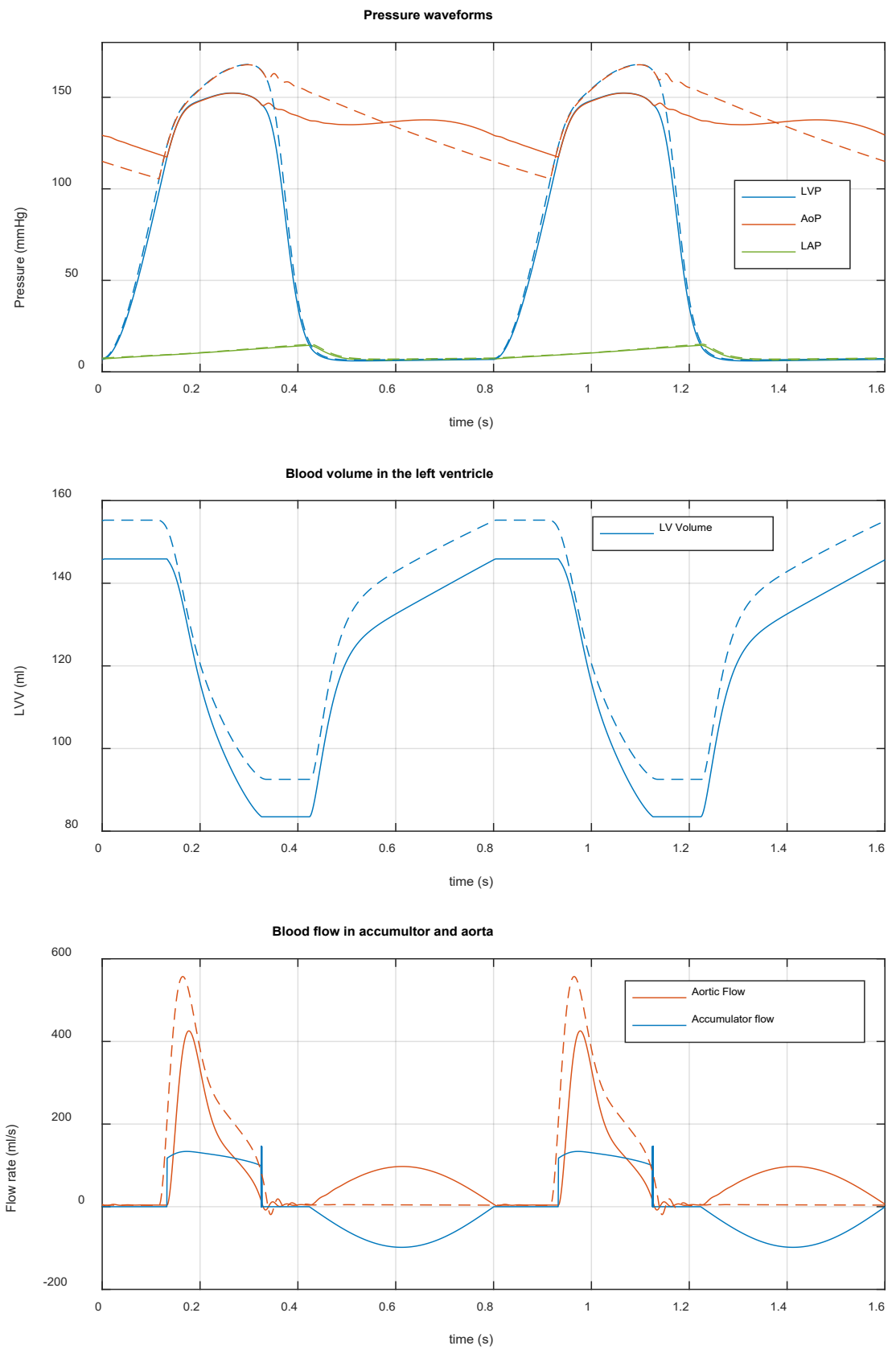

Figure 4.12. Waveforms for the hemodynamics of the unhealthy case (dashed lines) and the case of implementing the accumulator device idea with $R_{i}=1 \Omega$ (solid lines). 
Figure 4.13 and Figure 4.14 extend the comparison more by comparing the baseline with 3 other cases of different values for $R_{i}(5,1$, and $0.5 \Omega)$. Figure 4.13 demonstrates the changes in aortic pressure and flow, whereas Figure 4.14 demonstrates the changes in LV pressure, pressure build up inside the accumulator, and accumulator blood flow.
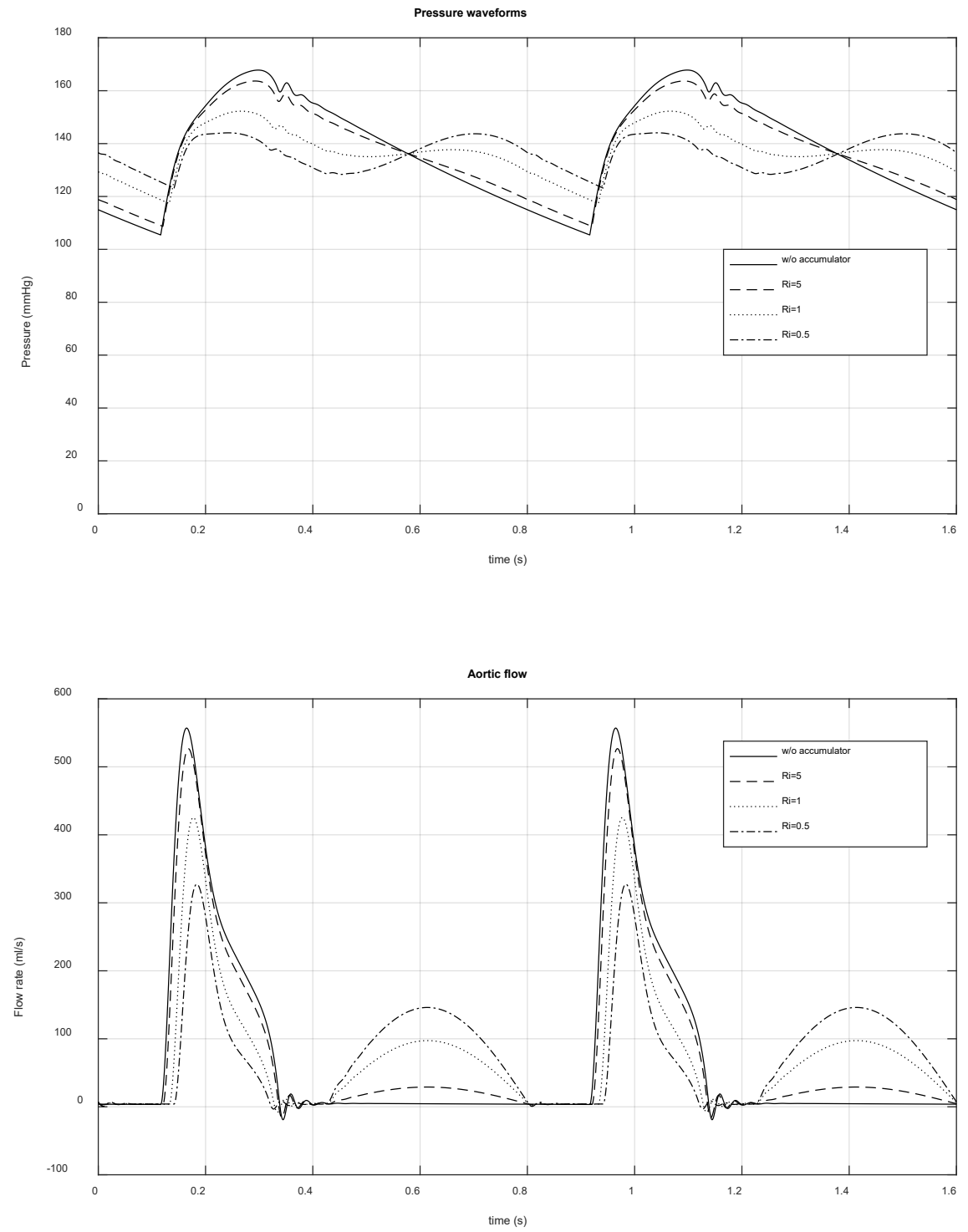

Figure 4.13. Aortic pressure and flow rate in the unhealthy case (w/o accumulator) and 3 cases representing the implementation of the accumulator device with different values for $R_{i}$. 

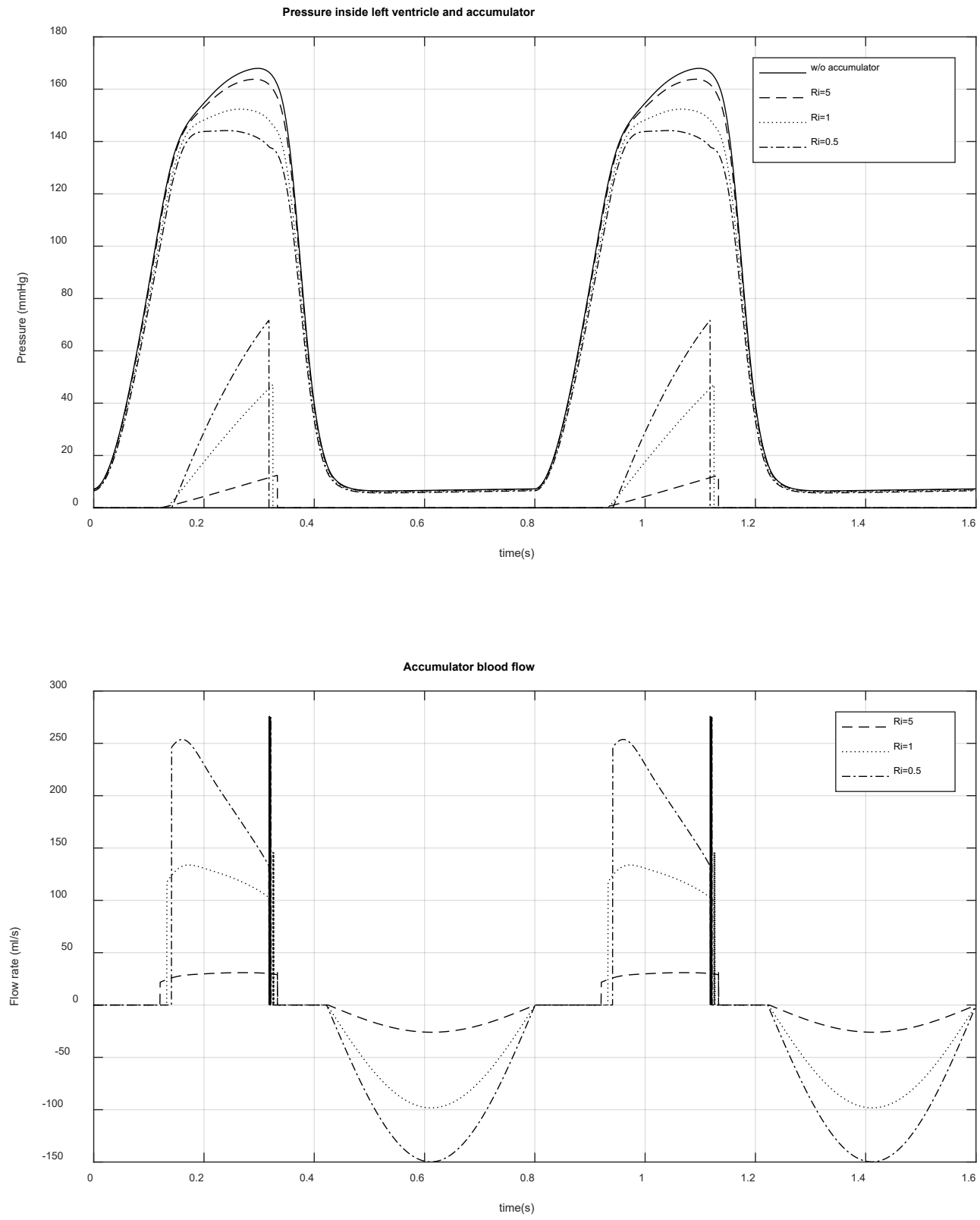

Figure 4.14. Accumulator influence on LV pressure and pressure build up inside the accumulator chamber (upper panel) and accumulator blood flow (lower panel). 
A more detailed comparison that can explain the direct influence of the selection of the value of $R_{i}$ is illustrated in Figure 4.15, Figure 4.16, and Figure 4.17, where the baseline condition (unhealthy case) is compared with 6 cases representing different values for $R_{i}$ $(5,2,1,0.75,0.5$, and $0.25 \Omega)$. Figure 4.15 demonstrates the influence of changing $R_{i}$ on the resulting aortic pressure, Figure 4.16 demonstrates the influence on LV pressure as well as the pressure inside the accumulator chamber, and Figure 4.17 demonstrates the influence on aortic flow and blood accumulation.

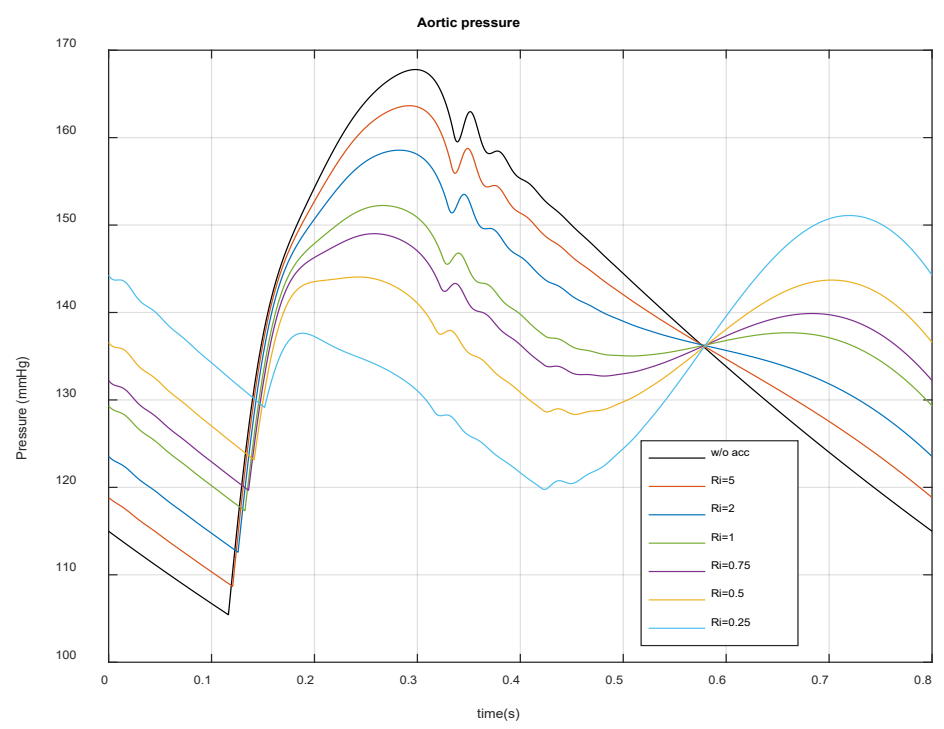

Figure 4.15. Influence of $R_{i}$ value on aortic pressure.

Note that the behavior becomes a little odd when $R_{i}$ becomes very small (i.e. $<0.5$ ). By looking at the aortic pressure waveform that corresponds to $R_{i}=0.25 \Omega$ in Figure 4.15, the diastolic pressure, unlike the pressure waveforms that correspond to greater $R_{i}$ values, is no longer equal to the pressure at the time of the aortic valve opening but rather, the pressure goes to lower values after the ejection phase, specifically during the isovolumic relaxation phase. This is because the amount of blood being ejected into the aorta prior to the onset of discharging the accumulator is very small. 


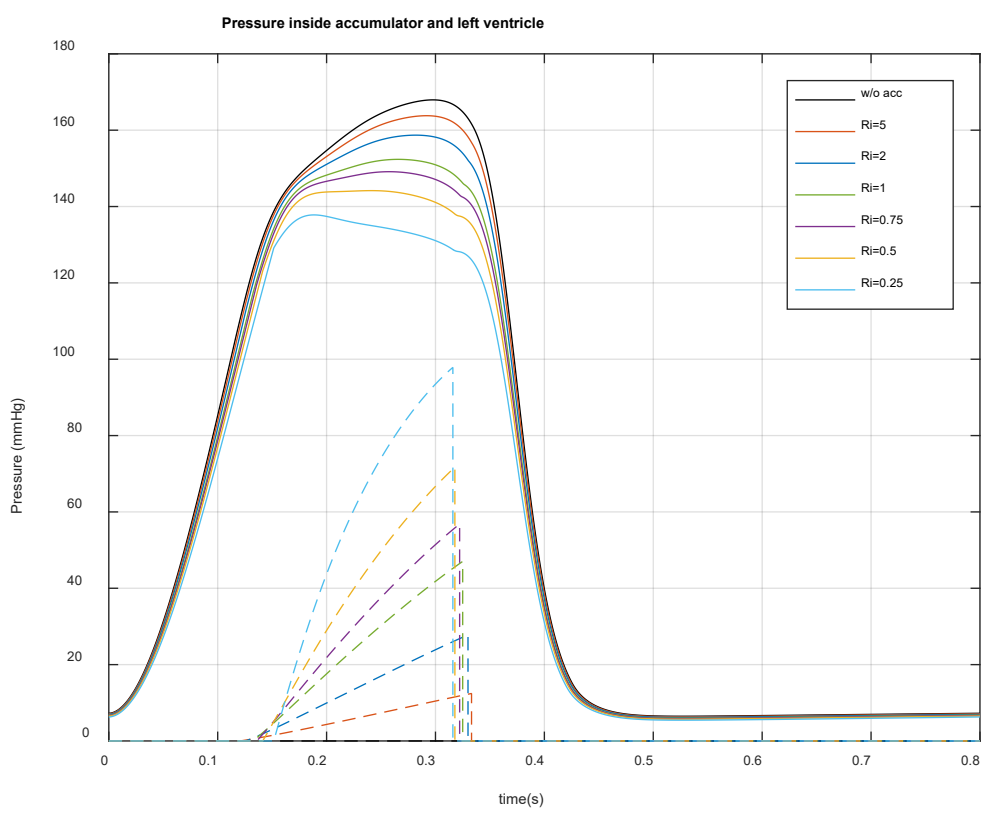

Figure 4.16. Influence of $R_{i}$ value on $\mathrm{LV}$ and accumulator pressures. Solid lines represent the left-ventricular pressure, whereas dashed lines represent the respective pressure inside the accumulator.

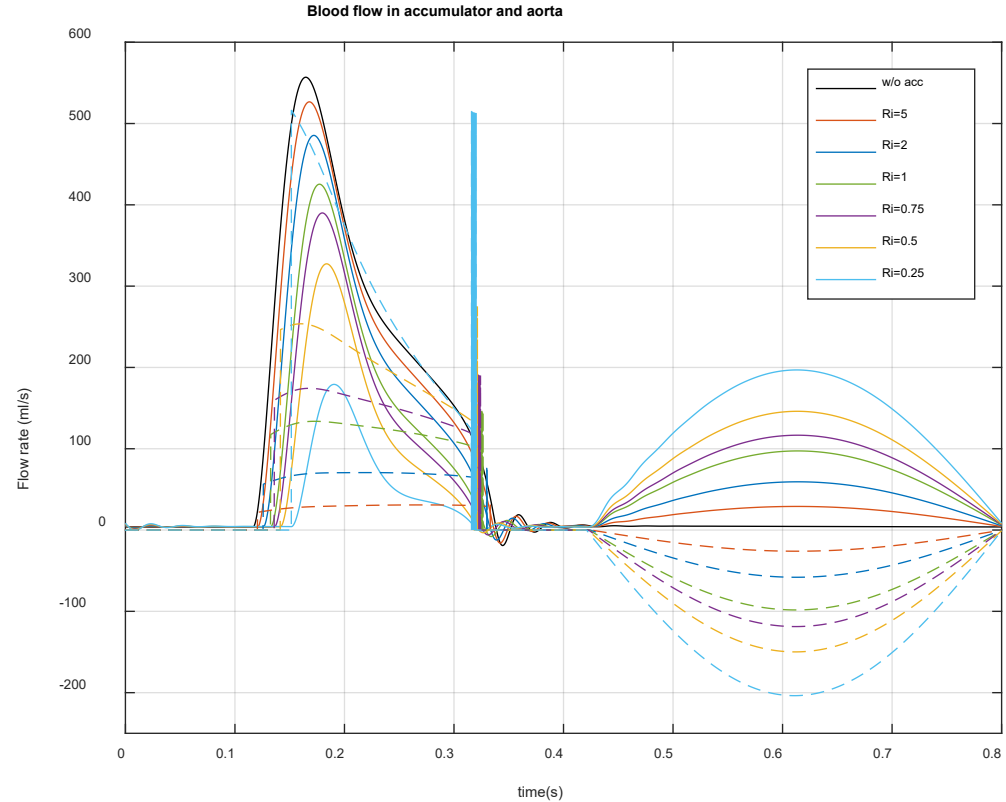

Figure 4.17. Influence of $R_{i}$ value on blood flow in the accumulator and aorta. Solid lines represent the blood flowing in the aorta (both through aortic valve and the accumulator discharge), whereas dashed lines represent the accumulator's blood flow (accumulation and discharging). 
Figure 4.18 shows a graphical demonstration for the influence of implementing the accumulator device on systolic, diastolic, and mean arterial pressures. As mentioned earlier, the systolic (diastolic) pressure decreases (increases) as $R_{i}$ decreases (i.e. blood accumulation increases); however, when $R_{i}$ becomes smaller than $0.5 \Omega$, the relationships are reversed.

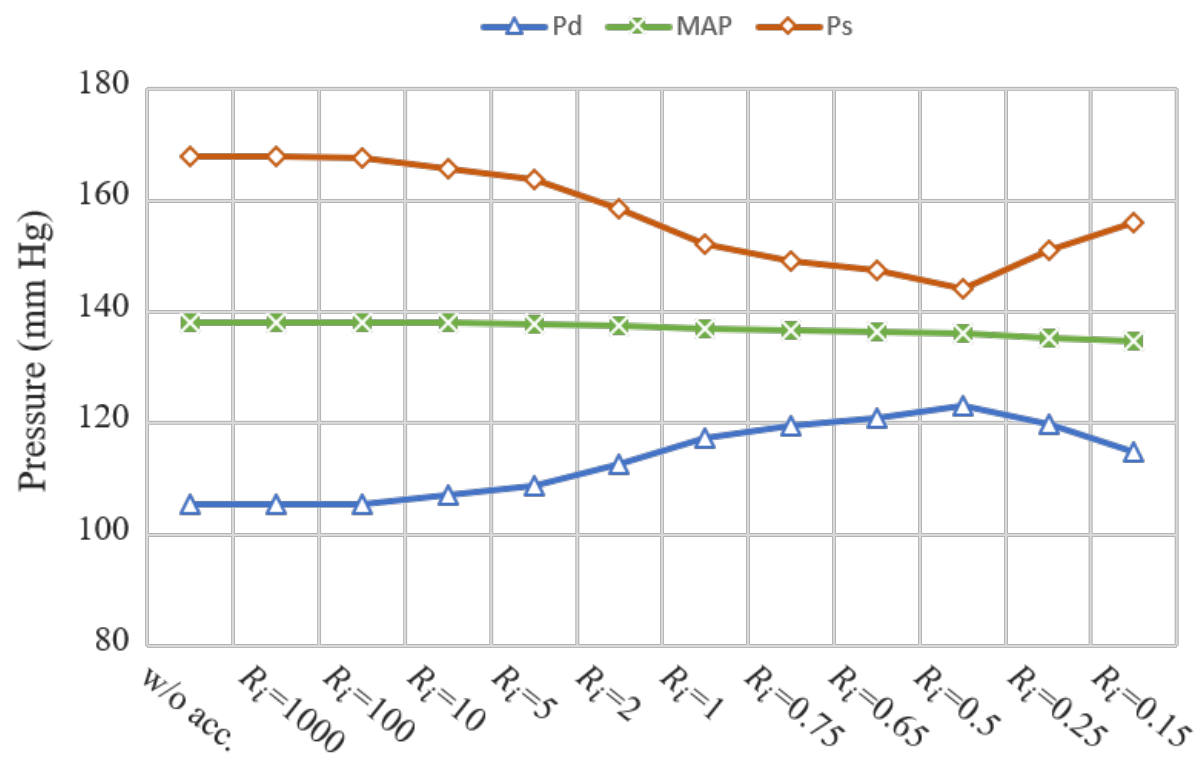

Accumulator inlet resistance $(\Omega)$

Figure 4.18. Influence of changing $R_{i}$ value on blood pressure.

Figure 4.19 illustrates another graphical relationship that demonstrates the influence of changing $R_{i}$ on the ratio of the amount of blood ejected through the aortic valve and the amount accumulated. The smaller the $R_{i}$, the more accumulation and less ejection through the aortic valve and vice versa. The graph in Figure 4.20 demonstrates another important relationship that relates between the amount of blood accumulation and pulse pressure. As $R_{i}$ decreases (equivalent to more blood accumulation), the pulse pressure (difference 
between systolic pressure and diastolic pressure) decreases. The relationship reverses at very small $R_{i}$ values (i.e. large values of blood accumulation).

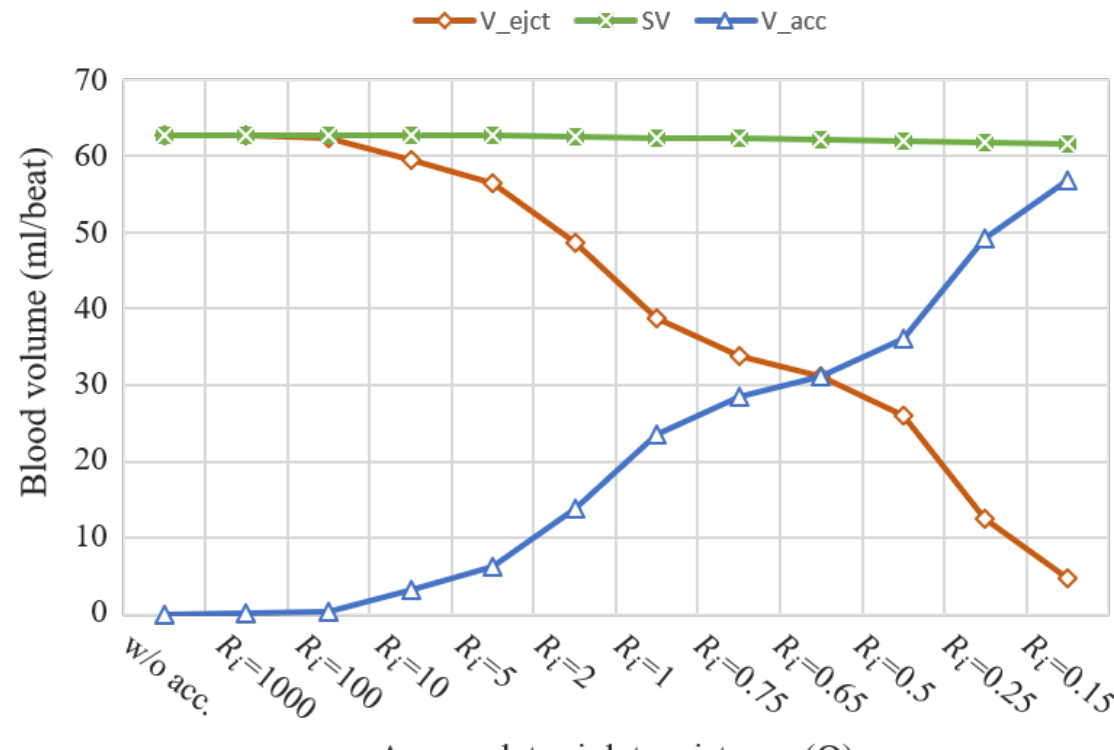

Accumulator inlet resistance $(\Omega)$

Figure 4.19. Influence of changing $R_{i}$ value on blood ejection.

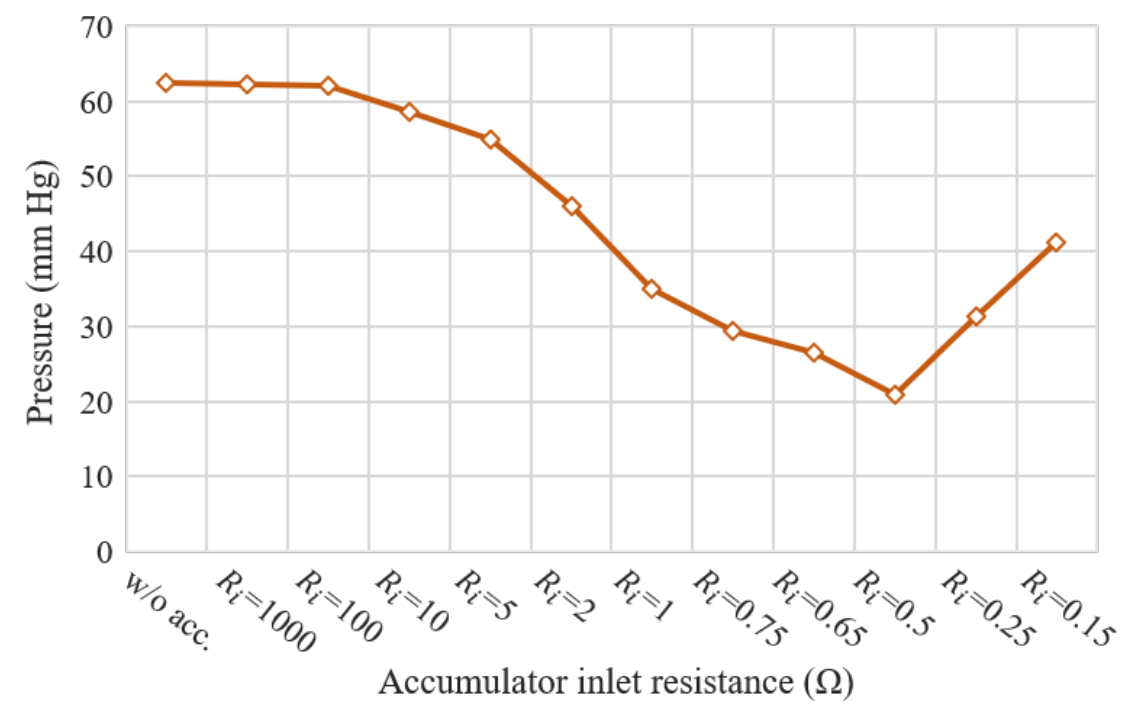

Figure 4.20. Influence of changing $R_{i}$ value on pulse pressure (PP). 
Figure 4.21 illustrates a relationship between $R_{i}$ and end-diastolic volume. As $R_{i}$ decreases (i.e. blood accumulation increases), EDV decreases; this could be because of increased accumulation that may cause some delay in the blood circulation during the cardiac cycle.

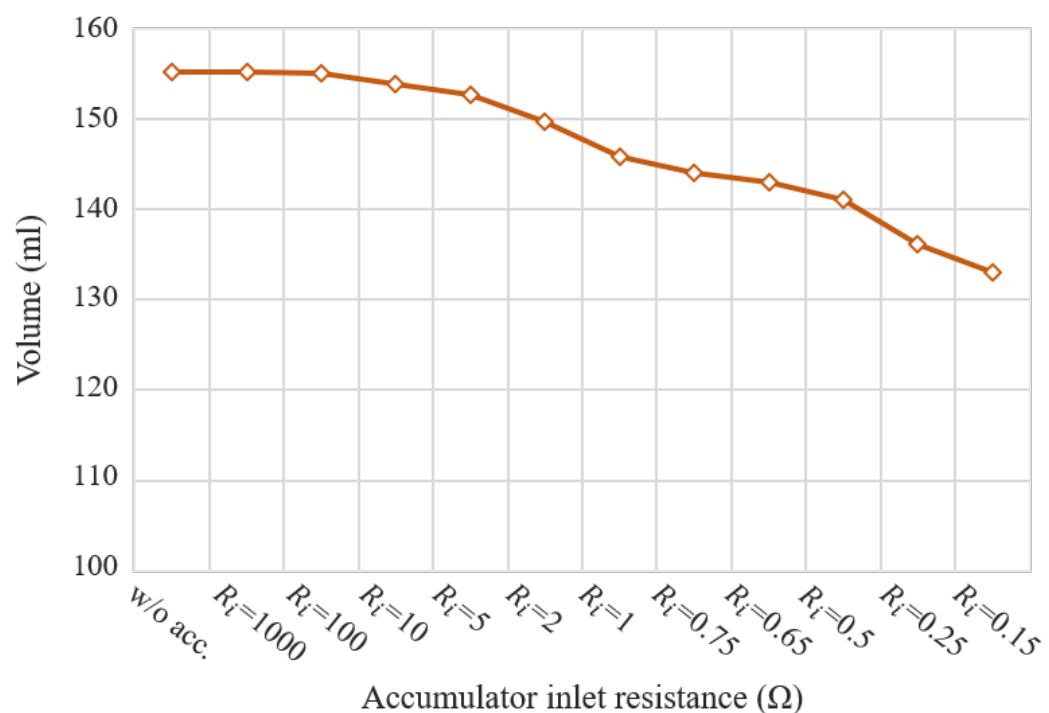

Figure 4.21. Influence of changing $R_{i}$ value on EDV.

\section{Accumulator Device Modulates the Duration of Cardiac Cycle Phases}

Another observation was recorded which relates to the influence of implementing the accumulator device on the duration of cardiac cycle phases. Table 4.5 and Figure 4.22 summarize the results for different values of $R_{i}$ as well as the baseline condition. It can be noticed that the ejection phase duration decreases as $R_{i}$ decreases. This is intuitive since as we decrease $R_{i}$, we allow for more accumulation (i.e. the additional path for blood flow through the accumulator inlet becomes of a smaller resistance). Accordingly, this accelerates the pressure drop inside the LV and consequently, shortens the ejection phase duration by shutting off the aortic valve sooner. On the other hand, the isovolumic contraction phase duration increased as $R_{i}$ decreases. This is also intuitive since as $R_{i}$ 
decreases, the diastolic pressure increases (Figure 4.18) which results in a longer wait time for the LVP to build up until it is sufficient to overcome the pressure in the aorta (the diastolic pressure), the moment when the aortic valve opens. The filling phase and isovolumic relaxation phase durations showed a slight increase as $R_{i}$ decreases.

\begin{tabular}{|c|c|c|c|c|c|}
\hline \multirow{2}{*}{$\begin{array}{c}\text { Accumulator } \\
\text { resistance } \\
\boldsymbol{R}_{\boldsymbol{i}}(\text { Ohms })\end{array}$} & \multicolumn{5}{|c|}{ Cardiac cycle phases duration (in seconds) } \\
\cline { 2 - 6 } & $\begin{array}{c}\text { Isovolumic } \\
\text { contraction }\end{array}$ & Ejection & $\begin{array}{c}\text { Isovolumic } \\
\text { relaxation }\end{array}$ & Filling & Total \\
\hline $\begin{array}{c}\text { without } \\
\text { accumulator }\end{array}$ & 0.111 & 0.219 & 0.091 & 0.379 & 0.8 \\
\hline $\mathbf{1 0 0 0}$ & 0.111 & 0.219 & 0.091 & 0.379 & 0.8 \\
\hline $\mathbf{1 0 0}$ & 0.112 & 0.218 & 0.091 & 0.379 & 0.8 \\
\hline $\mathbf{1 0}$ & 0.113 & 0.216 & 0.091 & 0.380 & 0.8 \\
\hline $\mathbf{5}$ & 0.115 & 0.213 & 0.092 & 0.380 & 0.8 \\
\hline $\mathbf{2}$ & 0.121 & 0.204 & 0.094 & 0.381 & 0.8 \\
\hline $\mathbf{1}$ & 0.127 & 0.193 & 0.098 & 0.382 & 0.8 \\
\hline $\mathbf{0 . 7 5}$ & 0.130 & 0.187 & 0.100 & 0.383 & 0.8 \\
\hline $\mathbf{0 . 6 5}$ & 0.132 & 0.183 & 0.102 & 0.383 & 0.8 \\
\hline $\mathbf{0 . 5}$ & 0.135 & 0.177 & 0.104 & 0.384 & 0.8 \\
\hline $\mathbf{0 . 2 5}$ & 0.146 & 0.164 & 0.105 & 0.385 & 0.8 \\
\hline $\mathbf{0 . 1 5}$ & 0.152 & 0.168 & 0.094 & 0.386 & 0.8 \\
\hline
\end{tabular}

Table 4.5. Influence of changing $R_{i}$ value on the duration of cardiac cycle phases. 


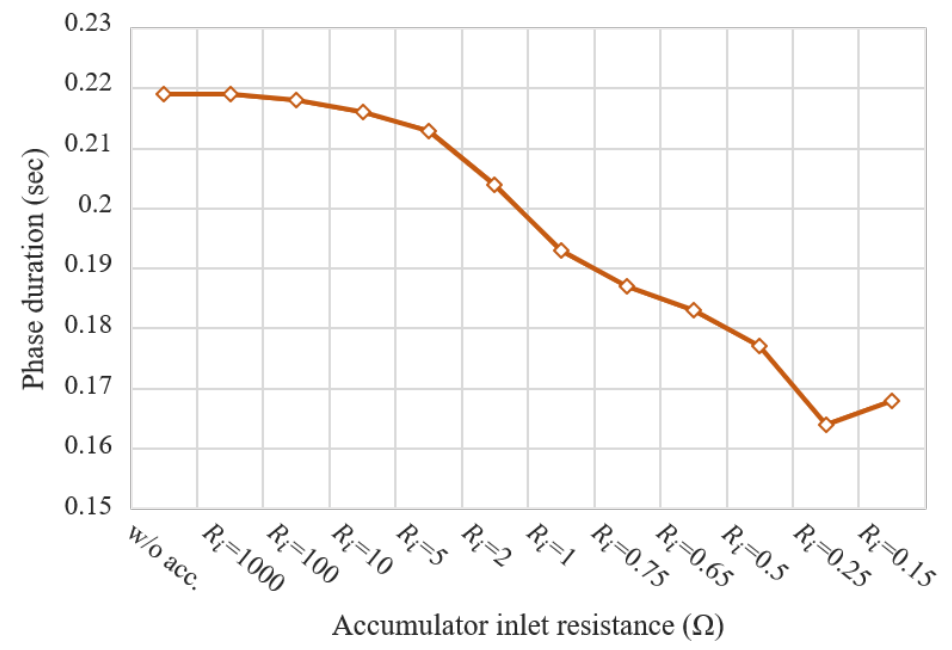

Figure 4.22. Influence of changing $R_{i}$ value on the duration of ejection phase.

\section{Testing Different Waveform Shapes of Flow Rate for Blood Discharged by the}

\section{Accumulator}

In this section, we investigate the response exhibited by the aorta in response to the shape of flow rate waveform for the blood that is discharged by the accumulator during the filling phase. As mentioned earlier, we are assuming in this section that the discharge flow rate $(I(t)$ in Figure 4.10) is known. This will serve as a preliminary analysis for the design of the controller that will drive the control input $U(t)$ (Figure 4.8 and Figure 4.9) in order to achieve a desired flow rate for blood discharge. In the following sections, we will investigate four different waveform profiles that are generated from well-known mathematical functions. To generate such waveforms, it is required that the actual volume of blood that was accumulated during ejection phase is known, and we will call this $V_{a c c}$. In addition to knowing $V_{a c c}$, the actual time interval during which the accumulator will discharge blood should also be known, we will refer to this as $\left[t_{0}, t_{f}\right]$, where $t_{0}$ and $t_{f}$ stand for the beginning and ending of discharging time, respectively. As discussed earlier, 
$t_{0}$ represents the onset of the filling phase of the cardiac cycle, whereas $t_{f}$ represents the end of the respective cardiac cycle. The generated flow rate signals were all based on a cardiac cycle duration of 0.8 seconds (corresponding to heart rate of 75 beats $/ \mathrm{min}$ ). The flow rate signals are generated such that the area under the curve is equal to $V_{a c c}$.

\section{Step function}

This is one of the simplest signal forms where the function is a constant value for all $t \in$ $\left[t_{0}, t_{f}\right]$. The step function signal of flow rate for a known $V_{a c c}$ looks like the following:

$$
F R(t)=\frac{V_{a c c}}{\left(t_{f}-t_{0}\right)}, \quad t_{0}<t<t_{f}
$$

\section{Trapezoidal function}

This function is somehow similar to the step function in the sense that it remains constant during almost the entire time interval, except for the short periods of time that follow $t_{0}$ and precedes $t_{f}$. Thus, it allows for a rising and falling time to switch between zero and the peak value and vice versa. This step is essential since it might not be practical to achieve a pure step function with the discontinuities occurring from the sudden sharp rise and fall as

in the step function. For the sake of simplicity, we will assume that the rising time and falling time are equal, and we will call this $t_{r}$. A typical trapezoidal function is illustrated in Figure 4.23. 


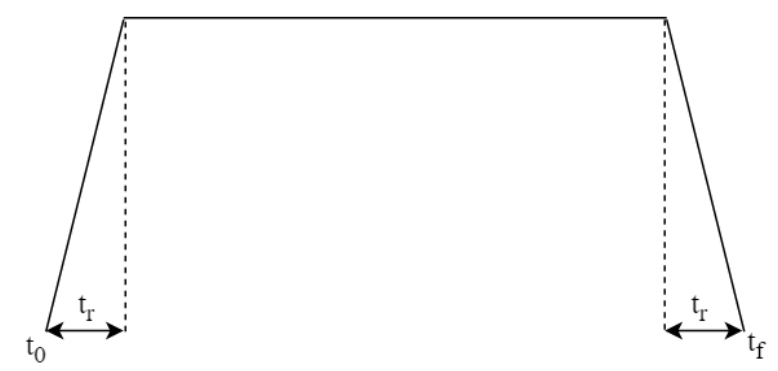

Figure 4.23. A typical shape for a trapezoidal function.

The trapezoid-function signal of blood discharge flow rate was generated according to the following formula:

$$
F R(t)=\left\{\begin{array}{lr}
\frac{\left(t-t_{0}\right)}{t_{r}} * \frac{V_{a c c}}{\left(t_{f}-t_{0}-t_{r}\right)}, & t_{0} \leq t<t_{0}+t_{r} \\
\frac{V_{a c c}}{\left(t_{f}-t_{0}-t_{r}\right)}, & t_{0}+t_{r} \leq t<t_{f}-t_{r} \\
\frac{V_{a c c}}{\left(t_{f}-t_{0}-t_{r}\right)} *\left[1-\frac{\left(t-t_{f}+t_{r}\right)}{t_{r}}\right], & t_{f}-t_{r} \leq t \leq t_{f}
\end{array}\right.
$$

\section{Sinusoidal function}

This is one of the most popular signal representations. The area under the curve of a positive half-period of a sine wave is:

$$
\int_{t_{0}}^{t_{f}} \sin \left(\frac{\pi\left(t-t_{0}\right)}{t_{f}-t_{0}}\right) d t=\frac{2 *\left(t_{f}-t_{0}\right)}{\pi}
$$

Thus, to generate a sine-wave signal of flow rate with an area under the curve being equal to $V_{a c c}$, the following formula is used:

$$
F R(t)=\frac{V_{a c c}}{\frac{2 *\left(t_{f}-t_{0}\right)}{\pi}} * \sin \left(\frac{\pi\left(t-t_{0}\right)}{t_{f}-t_{0}}\right), \quad t_{0}<t<t_{f}
$$




\section{Cycloidal function}

A typical cycloidal function is illustrated in Figure 4.24. The area under the arc of a cycloid, that starts at $t_{0}$ and ends at $t_{f}$, is $3 \pi r^{2}$, where $r$ represents the radius of the disk rolling in Figure 4.24 which is equal to $r=\frac{t_{f}-t_{0}}{2 \pi}$

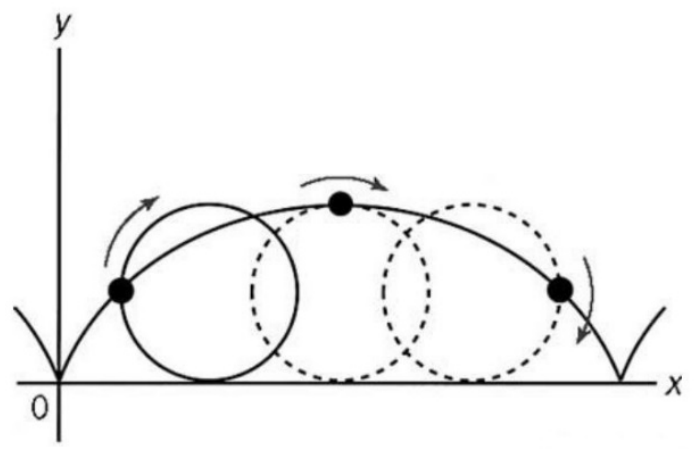

Figure 4.24. A typical shape for a cycloid.

Obtaining a cycloidal function is a little tricky since both the $\mathrm{x}$-axis variable (time) and $\mathrm{y}$ axis variable (flow rate) are functions of the angle $\theta$ which varies between 0 and $2 \pi$ according to the following equation:

$$
\left\{\begin{array}{lc}
t(\theta)=r(\theta-\sin \theta) & 0 \leq \theta \leq 2 \pi \\
F R(\theta)=\frac{V_{a c c}}{3 \pi r^{2}} * r(1-\cos \theta), & 0 \leq \theta \leq 2 \pi
\end{array}\right.
$$

However, the above equation is not sufficient to generate the desired cycloidal function of flow rate since we want to know the flow rate at a specific time (not angle) as our numerical simulation relies on exact steps of time. To resolve this issue, we used the numerical solver function in MATLAB to first solve for the angle $\theta$ that gives a specific time step and then plugging in this $\theta$ into the second equation of (4.28) to obtain the corresponding flow rate 
for that specific time (after making necessary rescaling such that the total area under the cycloidal curve is equal to the volume of accumulated blood to be discharged $V_{a c c}$ ).

Figure 4.25 demonstrates the generated step, trapezoidal, sinusoidal, and cycloidal flow rate signals to discharge an amount of blood accumulation of $27.4 \mathrm{ml}$, which corresponds to the case when $R_{i}=0.8 \Omega$. Figure 4.26, Figure 4.27, Figure 4.28, and Figure 4.29 respectively demonstrate the response of aortic pressure and flow due to applying a step, trapezoidal, sinusoidal, and cycloidal flow rate signal for blood that is discharged by the accumulator. We can notice that regardless of the shape of the flow rate signal, the systolic and diastolic pressures remained unaffected. The only difference is shown on the profile of the resulting aortic pressure and flow rate. The step function flow rate (Figure 4.26) caused a significant oscillatory behavior (ripples) in the resulting pressure and flow waveforms. This is possibly due to the response of the inductive element to the sudden sharp switching from zero to a peak value and vice versa. The other 3 waveform shapes performed well, with the sinusoidal and cycloidal flow rate signals resulting in smoother profiles in the respective aortic pressure waveforms compared to the one resulting from the trapezoidal signal. 


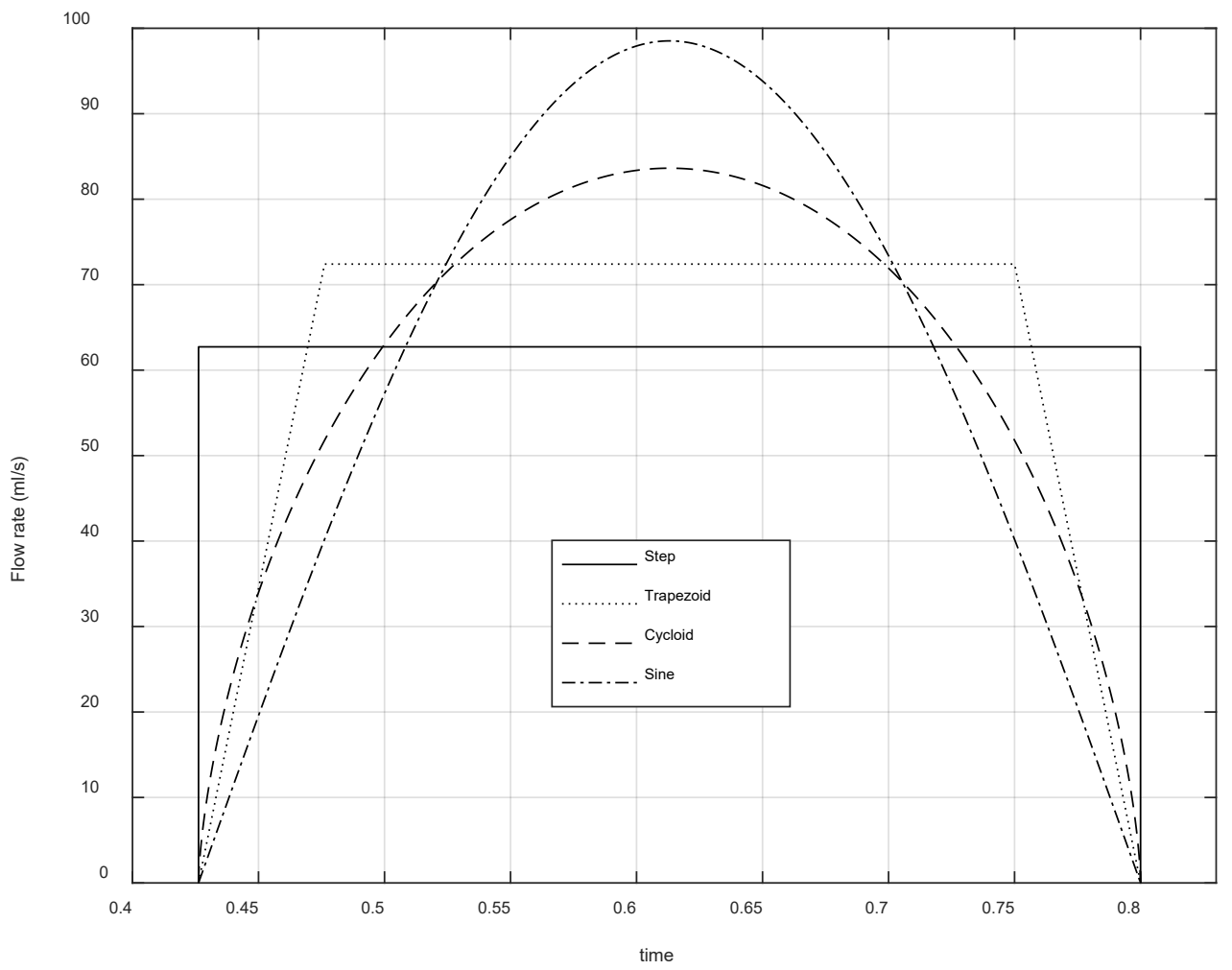

Figure 4.25. Generating the 4 signals of flow rate to discharge a blood accumulation of $27.4 \mathrm{ml}$ that corresponds to the case when $R_{i}=0.8 \Omega$. 

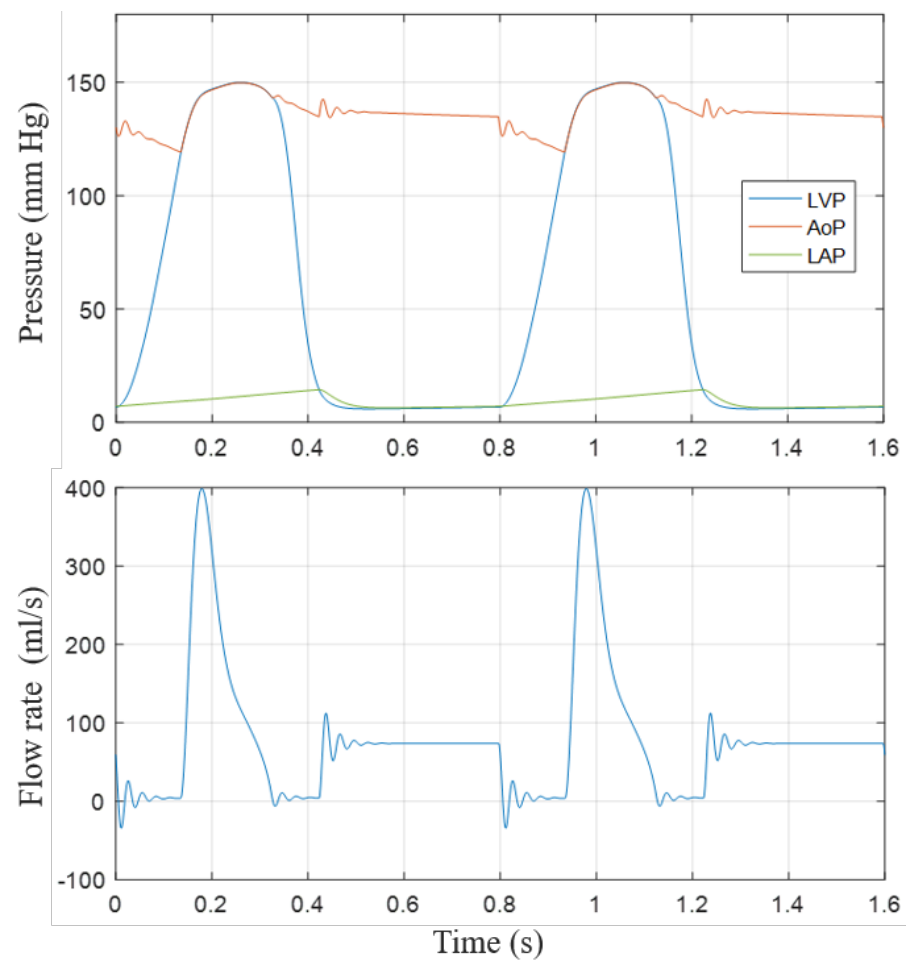

Figure 4.26. Response to a step function flow rate of discharged blood.
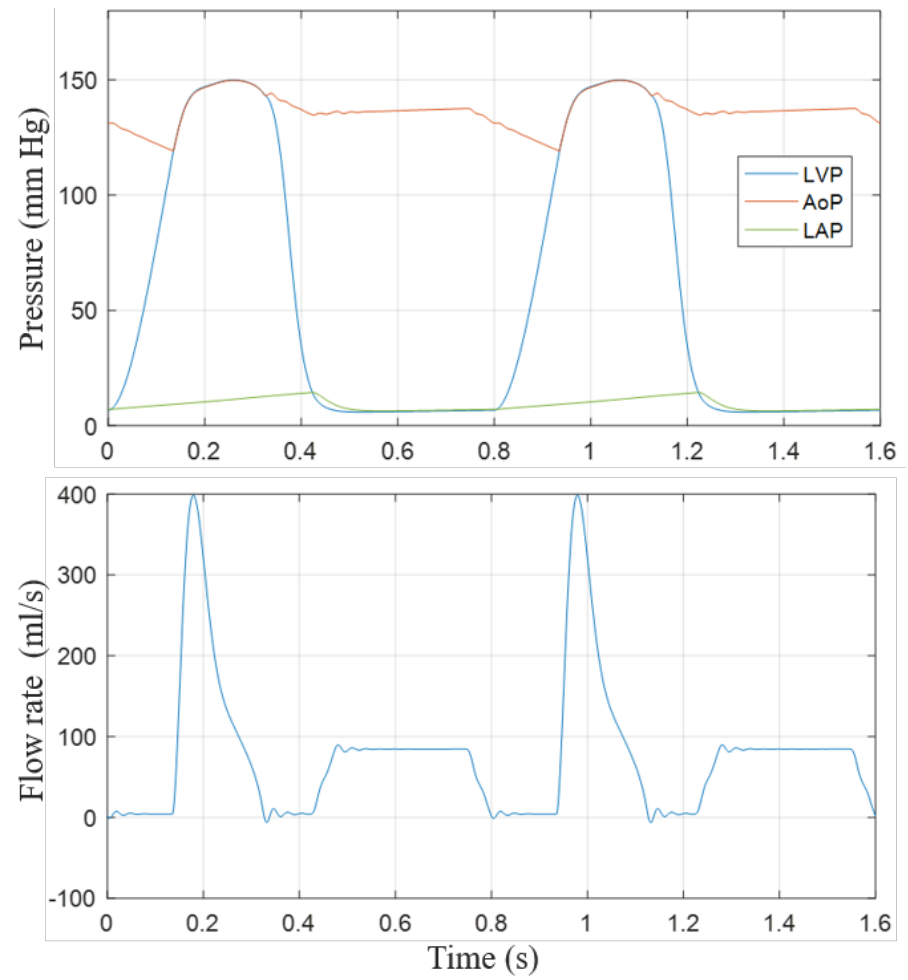

Figure 4.27. Response to a trapezoidal function flow rate of discharged blood. 

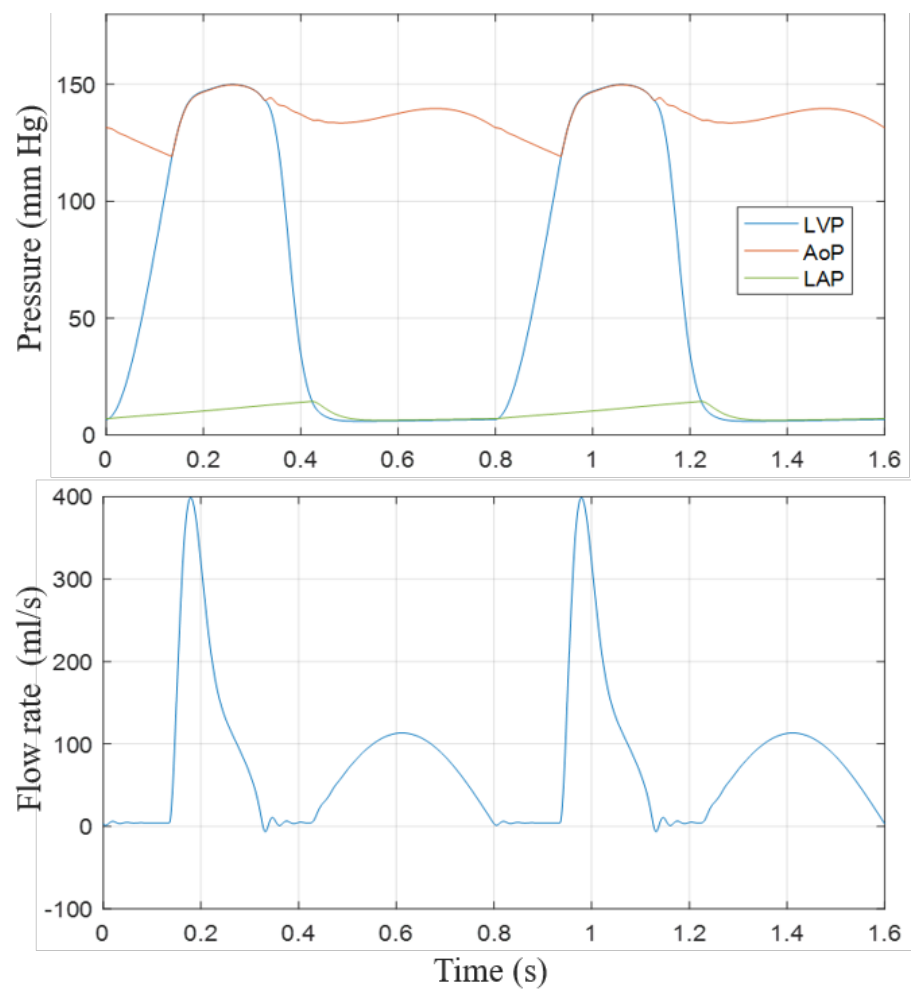

Figure 4.28. Response to a sinusoidal function flow rate of discharged blood.
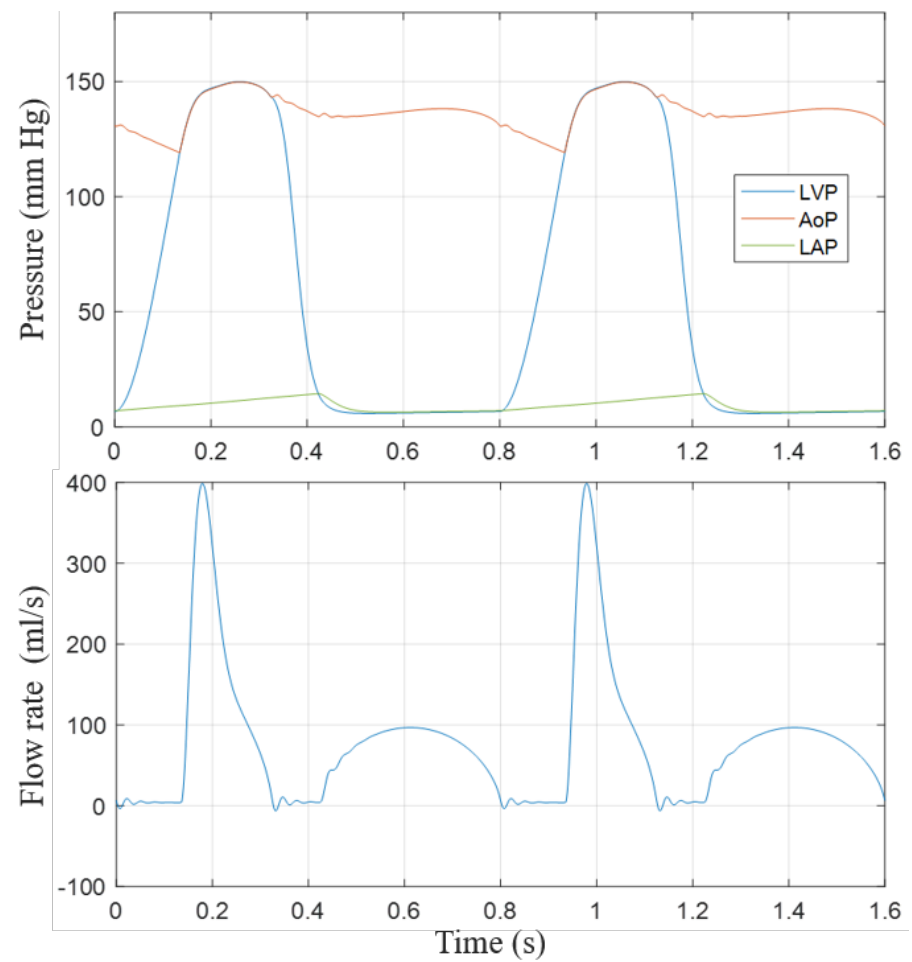

Figure 4.29. Response to a cycloidal-like function flow rate of discharged blood. 


\subsection{Discussion}

The modeling work related to implementing the accumulator device idea revealed promising results. The results in Table 4.3 indicate that the value of the accumulator's inlet resistance $\left(R_{i}\right)$, which governs the amount of blood accumulation, is the main determining factor influencing the hemodynamic changes when the accumulator device was incorporated with the cardiovascular system (Figure 4.3). The decrease in systolic pressure when $R_{i}$ was $0.5 \Omega$ compared to the systolic pressure of the unhealthy condition was significant, with a drop from 167.8 (in the unhealthy case) to $144.1 \mathrm{mmHg}$ (a decrease of $\sim 14 \%$ ). Although this caused a slight decrease in the cardiac output (from $4.7 \mathrm{~L} / \mathrm{min}$, in the unhealthy case, to $4.65 \mathrm{~L} / \mathrm{min}$ ), when the simulation was repeated with necessary adjustments in the initial conditions in order to achieve the same $\mathrm{CO}$ of $4.7 \mathrm{~L} / \mathrm{min}$, the systolic pressure still showed a significant decrease from 167.8 to $145.6 \mathrm{mmHg}$ (a decrease of $\sim 13 \%$ ), as shown in Table 4.4 . The diastolic pressure, on the other hand, showed to increase as $R_{i}$ decreases. This is expected since mean arterial pressure (MAP) is supposed to remain unchanged since there is no change occurring to the peripheral resistance and that the $\mathrm{CO}$ is unchanged, therefore, the decrease in systolic pressure is balanced by an increase in diastolic pressure. Another face for the influence of blood accumulation on the changes occurring to blood pressure is illustrated by the graph of Figure 4.20. The conclusion that can be drawn here is that the accumulator device basically works on reducing the pulse pressure, while maintaining MAP unchanged, much similar to the effect of adding artificial compliance that was discussed in chapter 3.

End-diastolic volume (EDV), on the other hand, also showed a decrease as the value of the accumulator's inlet resistance $\left(R_{i}\right)$ was decreased (i.e. blood accumulation is 
increased). We think that this is an important insight. With the fact that the contractility characteristics of the LV (such as the contractility index $E_{\mathrm{es}}$ ) are unchanged, the addition of the new path created by the accumulator for the blood that is pumped out of the LV due to contraction is expected to cause an increase in CO. However, this did not happen as the CO remained almost constant (minimally decreased as $R_{i}$ decreased and blood accumulation increased). We predict that the decrease in EDV is the balancing factor in this phenomena since, according to Frank-Starling law, as the end-diastolic volume decreases, the stretching in the LV muscle decreases which results in a lesser contraction force and consequently, a lesser amount of blood ejection. Therefore, the addition of an extra path for the blood leaving the LV was compensated by a decrease in EDV and consequently, the resulting $\mathrm{CO}$ was kept almost unchanged.

The implementation of the accumulator device also showed an influence on modulating the duration of cardiac cycle phases (Table 4.5 and Figure 4.22). The ejection phase duration was decreased as $R_{i}$ decreased (blood accumulation increased); this is possibly due to the addition of a new path for blood flow that is created by the accumulator, which is expected to accelerate emptying the LV and consequently, lowering its pressure faster which should cause the aortic valve to close faster. On the other hand, the duration of the isovolumic contraction phase was increased as $R_{i}$ decreased (blood accumulation increased). This is possibly due to the increase in diastolic pressure as $R_{i}$ is decreased, which means that the LV will need to wait for a longer time until the pressure inside its chamber increases sufficiently such that to exceed the pressure in the aorta (which is the diastolic pressure in normal cases) for the aortic valve to open. 
Since our control input $U(t)$ (Figure 4.3), or pressure pump $F$ (Figure 4.1), is the force that can control the profile of flow rate for the blood that is discharged by the accumulator, we investigated the response due to the application of given different waveform shapes of flow rate. The step-function flow rate caused significant oscillatory behavior (ripples) in the resulting aortic pressure waveform, most possibly resulting from the inductive component that represents blood inertia, due to the instant sharp jump from zero to a peak level of flow rate and vice versa. This gives an impression that the step-like function of flow rate is not desirable for the control input to achieve when discharging the accumulator. The other three functions (trapezoidal, sinusoidal, and cycloidal) all performed well compared to the step function signal. 


\section{CHAPTER 5 Controller Design}

The implementation of the accumulator concept requires a controller to regulate the process of discharging the accumulated blood back to the body. It is crucial that the whole amount of accumulated blood is re-delivered to the aorta within a specified time frame, which we decided during the filling phase of the cardiac cycle. This is essential for two reasons: to make sure that the stroke volume is not affected and to avoid any accumulation build up by emptying the accumulator completely prior to the onset of the next cardiac cycle accumulation. In addition, it is of great importance that the accumulated blood is not discharged at a random flow rate but rather follows a trajectory of an optimal desired flow rate.

In this chapter, we investigate the design of a controller that can meet the requirements mentioned above. Optimal control theory represents a mature mathematical discipline that can be applied to solve numerous engineering applications. We will apply the linear quadratic tracking (LQT) technique to design a controller that can maintain a flow rate for discharged blood as close as possible to a desired flow rate trajectory. It is important to remember that we are dealing with a time-varying system of the cardiovascular model that is presented in Figure 4.3, and so one should appreciate the existence of such modern control techniques to solve such kind of problems, where the use of classical control theory might be associated with some complications. 


\subsection{Introduction}

Optimal control was born in 1697 (over 300 years ago) when Johann Bernoulli, the great mathematician, published his solution to the brachystochrone problem [65]. The roots of optimal control theory originate from calculus of variations which was developed during the $16^{\text {th }}$ and $17^{\text {th }}$ century [66]. L. S. Pontryagin was behind the most important contribution to optimal control systems in his work of the maximum principle in 1956 [67]. In this chapter, we apply the linear quadratic tracking (LQT) technique to maintain the output (the actual flow rate of discharged blood from accumulator device) as close as possible to a desired flow rate. The cardiovascular system model with the accumulator device (Figure 4.3) was described according to the following linear, time-varying, system:

$$
\begin{aligned}
\dot{x}(t) & =A(t) x(t)+B(t) u(t) \\
y(t) & =C(t) x(t)+D(t) u(t)
\end{aligned}
$$

Our system is a single input single output (SISO) one, where $x(t)$ is the $6^{\text {th }}$ order state vector (state variables are listed in Table 4.2), $u(t)$ is the input (represented by $U(t)$ in Figure 4.3), and $y(t)$ is the output (actual flow rate of blood discharged from the accumulator). The vectors $B(t)$ and $C(t)$ and the scalar $D(t)$ are time invariant, and the state matrix $A(t)$ is the only time varying component in our system, where this time variance is arising from the time-varying elastance function of the left ventricle (Figure 3.4). Thus, for the sake of simplicity, we can re-write the system in (5.1) as follows:

$$
\begin{aligned}
& \dot{x}(t)=A(t) x(t)+B u(t) \\
& y(t)=C x(t)+d u(t)
\end{aligned}
$$




\subsection{Methodology}

The objective here is to control the system in Figure 4.3, which is described by the equations in (5.2), such that the output $y(t)$ tracks a desired output $z(t)$ with the least possible error during the interval $\left[t_{0}, t_{f}\right] . z(t)$ here represents a desired waveform profile for the flow rate of the blood that is discharged by the accumulator device. The procedure used to solve this linear quadratic tracking (LQT) problem followed the one presented in [66], which is called the Pontryagin Minimum Principle; however, the derivation work listed below was made by the author of this document from scratch. The equations in [66] were derived based on a system that has no feedforward term $D(t)$, unlike our system which includes this term as shown in equations (4.22) and (5.1). The reader is referred to the appendix for more details related to the following derivation work.

We start by defining the error $e(t)$ according to the following:

$$
e(t)=z(t)-y(t)
$$

Note that $e(t)$ is scalar since we are dealing with a single input single output system.

We then choose our performance index according to the following:

$$
J=\frac{1}{2} e^{T}\left(t_{f}\right) F\left(t_{f}\right) e\left(t_{f}\right)+\frac{1}{2} \int_{t_{0}}^{t_{f}}\left[e^{T}(t) Q(t) e^{T}(t)+u^{T}(t) R(t) u(t)\right] d t
$$

where $Q(t)$ and $R(t)$ are called the error weighted coefficient and control weighted coefficient, respectively. $F\left(t_{f}\right)$ is called the terminal cost weighted coefficient. Since $Q(t)$ and $R(t)$ are scalar functions that will be chosen to be constant values, then $Q(t), R(t)$, 
and $F\left(t_{f}\right)$ are represented next by the scalars $q, r$, and $f$, respectively. Equation (5.4) can now be simplified more since all its variables are scalars according to the following:

$$
J=\frac{1}{2} f\left[e\left(t_{f}\right)\right]^{2}+\frac{1}{2} \int_{t_{0}}^{t_{f}}\left(q[e(t)]^{2}+r[u(t)]^{2}\right) d t
$$

The error in equation (5.3) can be re-written as follows:

$$
e(t)=z(t)-C x(t)-d u(t)
$$

Now we can apply the Pontryagin Minimum Principle according to the steps presented next:

- Step 1: Formulating the Hamiltonian function:

$$
\begin{aligned}
H(x(t), u(t), \lambda(t)) & \\
& =\frac{1}{2} q[z(t)-C x(t)-d u(t)]^{2}+\frac{1}{2} r[u(t)]^{2} \\
& +\lambda^{T}(t)[A(t) x(t)+B u(t)]
\end{aligned}
$$

where $\lambda(t)$ is called the co-state vector, the superscript " ()$^{T}$ " denotes the transpose of a matrix.

- Step 2: Finding the open-loop optimal control from the Hamiltonian:

$$
\frac{d H}{d u}=0 \longrightarrow q *(-d) *[z(t)-C x(t)-d u(t)]+r u(t)+B^{T} \lambda(t)=0
$$


We can now write the equation for the optimal control:

$$
u^{*}(t)=\frac{-1}{r+q d^{2}}\left[q d C x^{*}(t)+B^{T} \lambda^{*}(t)-q d z(t)\right]
$$

the superscript " ()$^{*}$ " means "optimal".

- Step 3: Finding the state and co-state systems from the Hamiltonian:

$$
\dot{x}(t)=\frac{d H}{d \lambda}=A(t) x(t)+B u(t)
$$

Plugging in the optimal control in (5.9) into (5.10), the optimal state equation becomes:

$$
\dot{x}^{*}(t)=\left[A(t)-\frac{q d}{r+q d^{2}} B C\right] x^{*}(t)-\frac{B B^{T}}{r+q d^{2}} \lambda^{*}(t)+\frac{d q}{r+q d^{2}} B z(t)
$$

The optimal co-state equation can now be found from the Hamiltonian (5.7) according to the following:

$$
\begin{aligned}
\dot{\lambda}^{*}(t)=-\frac{d H}{d x} & =\left(\frac{q^{2} d^{2}}{r+q d^{2}} C^{T} C-q C^{T} C\right) x^{*}(t) \\
& +\left(\frac{q d}{r+q d^{2}} C^{T} B^{T}-A^{T}(t)\right) \lambda^{*}(t)+\left(q C^{T}-\frac{q^{2} d^{2}}{r+q d^{2}} C^{T}\right) z(t)
\end{aligned}
$$

For the sake of simplicity, let us define:

$$
\begin{aligned}
& \alpha=\frac{q d}{r+q d^{2}} \\
& \beta=\frac{q^{2} d^{2}}{r+q d^{2}} \\
& V=\frac{1}{r+q d^{2}} B^{T}
\end{aligned}
$$


Combining the optimal state equation (5.11) and optimal co-state equation (5.12) and using the relations defined in (5.13), we can now obtain the Hamiltonian canonical system:

$$
\left[\begin{array}{c}
\dot{x}^{*}(t) \\
\dot{\lambda}^{*}(t)
\end{array}\right]=\left[\begin{array}{cc}
A(t)-\alpha B C & -B V \\
(\beta-q) C^{T} C & \alpha C^{T} B^{T}-A^{T}(t)
\end{array}\right]\left[\begin{array}{l}
x^{*}(t) \\
\lambda^{*}(t)
\end{array}\right]+\left[\begin{array}{c}
\alpha B \\
(q-\beta) C^{T}
\end{array}\right] z(t)
$$

The above system includes $2 n$ differential equations (12 differential equations in our case since our cardiovascular system has 6 state variables).

The boundary conditions of the Hamiltonian canonical system in (5.14) are given by the initial condition of the states:

$$
x\left(t=t_{0}\right)=x\left(t_{0}\right)
$$

and the final condition comes from the co-states, where the final time $t_{f}$ being specified, according to the following:

$$
\begin{aligned}
\lambda\left(t_{f}\right)=\frac{\partial}{\partial x\left(t_{f}\right)} & {\left[\frac{1}{2} f\left(t_{f}\right)\left[e\left(t_{f}\right)\right]^{2}\right]=\frac{\partial}{\partial x\left(t_{f}\right)}\left[\frac{1}{2} f\left[z\left(t_{f}\right)-C x\left(t_{f}\right)-d u\left(t_{f}\right)\right]^{2}\right] } \\
& =-f C^{T}\left[z\left(t_{f}\right)-C x\left(t_{f}\right)-d u\left(t_{f}\right)\right] \\
& =f C^{T} C x\left(t_{f}\right)+f C^{T} d u\left(t_{f}\right)-f C^{T} z\left(t_{f}\right)
\end{aligned}
$$

Plugging in the expression for $u^{*}(t)$ in (5.9) into the above equation, we get:

$$
\begin{gathered}
\lambda^{*}\left(t_{f}\right)=\left(I+f d C^{T} V\right)^{-1} f(1-\alpha d) C^{T} C x^{*}\left(t_{f}\right) \\
-\left(I+f d C^{T} V\right)^{-1} f(1-\alpha d) C^{T} z\left(t_{f}\right)
\end{gathered}
$$

where $I$ is the identity matrix of size $6 \times 6$. 
- Step 4: Finding Riccati and Vector equations: the co-state boundary condition in (5.16) and the solution of the Hamiltonian canonical system (5.14) indicate that there is a relationship between the state and co-state according to the following:

$$
\lambda^{*}(t)=P(t) x^{*}(t)-g(t)
$$

where $P(t)$ is an $n \times n$ symmetric matrix (6x6 in our cardiovascular system model) and $g(t)$ is an $n \times 1$ vector ( $6 \times 1$ in our case), both need to be determined so as to satisfy the system in (5.14). This can be accomplished by deriving two key equations called differential Riccati equation (DRE) and vector differential equation, which will be shown next.

Starting with differentiating (5.17) with respect to time, we obtain:

$$
\dot{\lambda}^{*}(t)=\dot{P}(t) x^{*}(t)+P(t) \dot{x}^{*}(t)-\dot{g}(t)
$$

Substituting for $\dot{x}^{*}(t)$ and $\dot{\lambda}^{*}(t)$ from (5.14) and for $\lambda^{*}(t)$ from (5.17) into (5.18) lead to the following two important relationships:

$$
\begin{gathered}
\dot{P}(t)=P(t)(\alpha B C-A(t)+B V P(t))+(\beta-q) C^{T} C+\left(\alpha C^{T} B^{T}-A^{T}\right) P(t) \\
\dot{g}(t)=\left(P(t) B V+\alpha C^{T} B^{T}-A^{T}\right) g(t)+\left(\alpha P(t) B-q C^{T}+\beta C^{T}\right) z(t)
\end{gathered}
$$

where equation (5.19) is called the matrix differential Riccati equation (DRE) and equation (5.20) is called the vector differential equation. 
Since $P(t)$ is symmetric, equations (5.19) and (5.20) result in a set of $n(n+1) / 2+n$ first-order differential equations with the following boundary conditions:

$$
\begin{gathered}
P\left(t_{f}\right)=\left(I+f d C^{T} V\right)^{-1} f(1-\alpha d) C^{T} C \\
g\left(t_{f}\right)=\left(I+f d C^{T} V\right)^{-1} f(1-\alpha d) C^{T} z\left(t_{f}\right)
\end{gathered}
$$

Therefore, the $n(n+1) / 2+n$ first-order differential equations (21 equations in our case) resulting from the matrix DRE (5.19) and the vector differential equation (5.20) can be solved backward using the boundary conditions in (5.21) and (5.22).

- Step 5: Finding the closed-loop optimal control by plugging in the optimal co-state (5.17) into (5.9), we obtain:

$$
u^{*}(t)=-\frac{1}{r+q d^{2}}\left[q d C x^{*}(t)+B^{T}\left(P(t) x^{*}(t)-g(t)\right)-q d z(t)\right]
$$

- Step 6: Finding the optimal state by plugging in the optimal control (5.23) into the state equation (5.10), we obtain:

$$
\begin{gathered}
\dot{x}^{*}(t)=\left(A(t)-\frac{q d}{r+q d^{2}} B C-\frac{1}{r+q d^{2}} B B^{T} P(t)\right) x^{*}(t) \\
+\frac{1}{r+q d^{2}} B B^{T} g(t)+\frac{q d}{r+q d^{2}} B z(t)
\end{gathered}
$$

It can be noted that computing the Riccati coefficient matrix $P(t)$ does not depend on the desired output $z(t)$. Once the problem is specified in terms of the plant matrices $A(t)$, $B(t), C(t)$, and $D(t)$, the final time $t_{f}$, and the cost functional coefficients $f\left(t_{f}\right), q(t)$, 
and $r(t)$, the matrix function $P(t)$ can be completely computed offline. On the other hand, computing the vector $g(t)$ depends on the desired output $z(t)$.

Figure 5.1 demonstrates a block diagram for the plant (5.2) and the closed-loop optimal control (5.23).

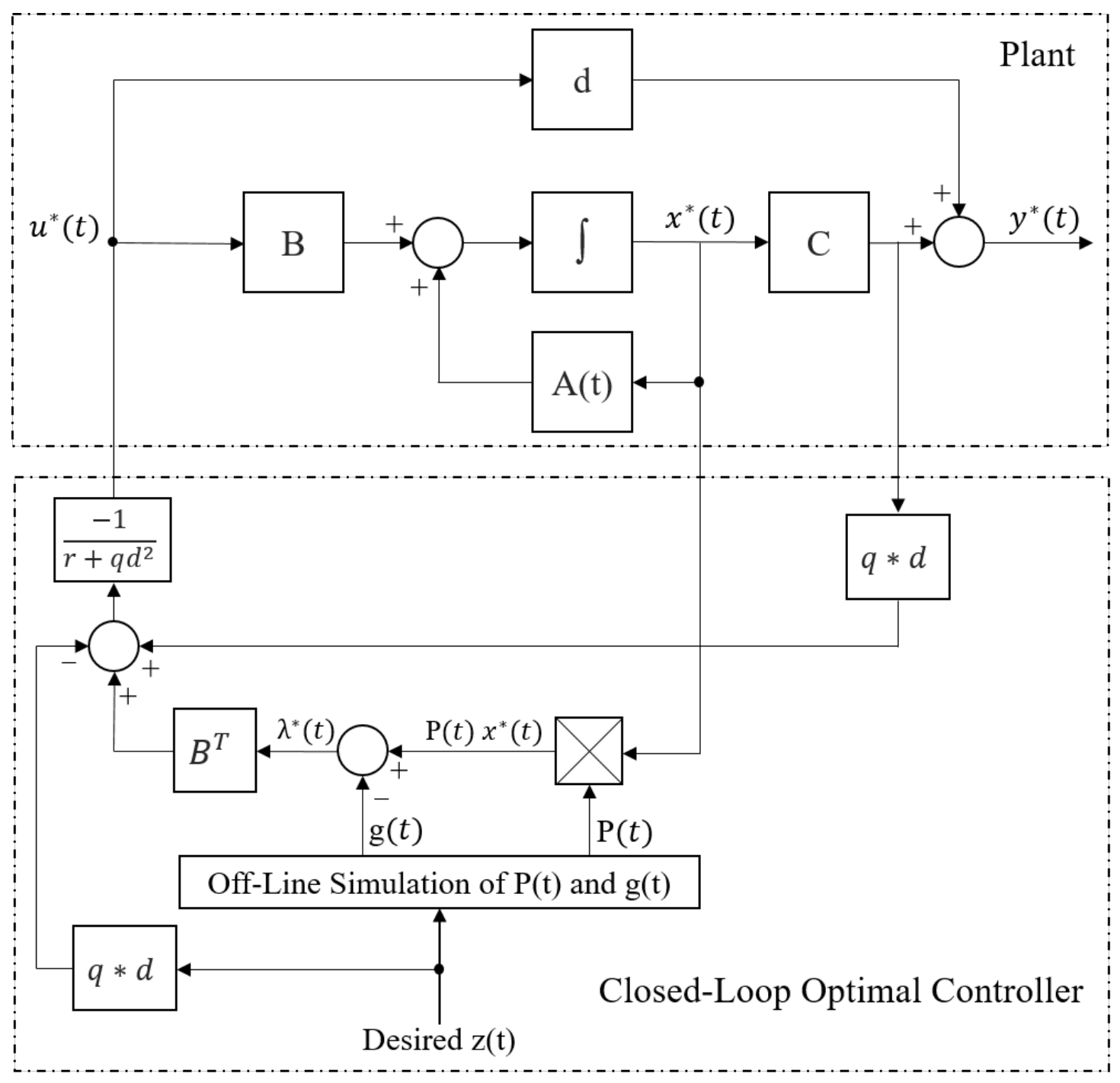

Figure 5.1. Implementation of the tracking system optimal controller. 


\subsection{Simulation Results and Discussion}

The four generated signals of flow rate in Figure 4.25 will now be considered as

desired signals, $z(t)$ that we want our control input $U(t)$ of Figure 4.9 to generate. We will apply the linear quadratic tracking (LQT) technique that was discussed earlier and demonstrated in Figure 5.1 to achieve such desired signals. This requires tuning the ratio of $Q$ and $R$ (the error and control weighted coefficients, respectively), where our concern here is to penalize the error more. Figure 5.2 illustrates the performance of the optimal controller in tracking the four signals of flow rate, that were presented in Figure 4.25, for different $Q / R$ ratios $(10,100$, and 1000). It can be noted that when the ratio is small (such as 10), the performance is poor, whereas when $Q / R$ is large (such as 1000), the actual signal generated by the optimal controller almost overlapped the desired one. Table 5.1 presents a more detailed performance comparison. It can be noted that a $Q / R$ ratio of 1 resulted in a back flow of $11.71 \mathrm{ml}$ (i.e. negative flow, however this should not occur if we used unidirectional check valves). In contrast, at extremely large $Q / R$ ratios, the accumulator completely discharged the whole amount of accumulated blood. 

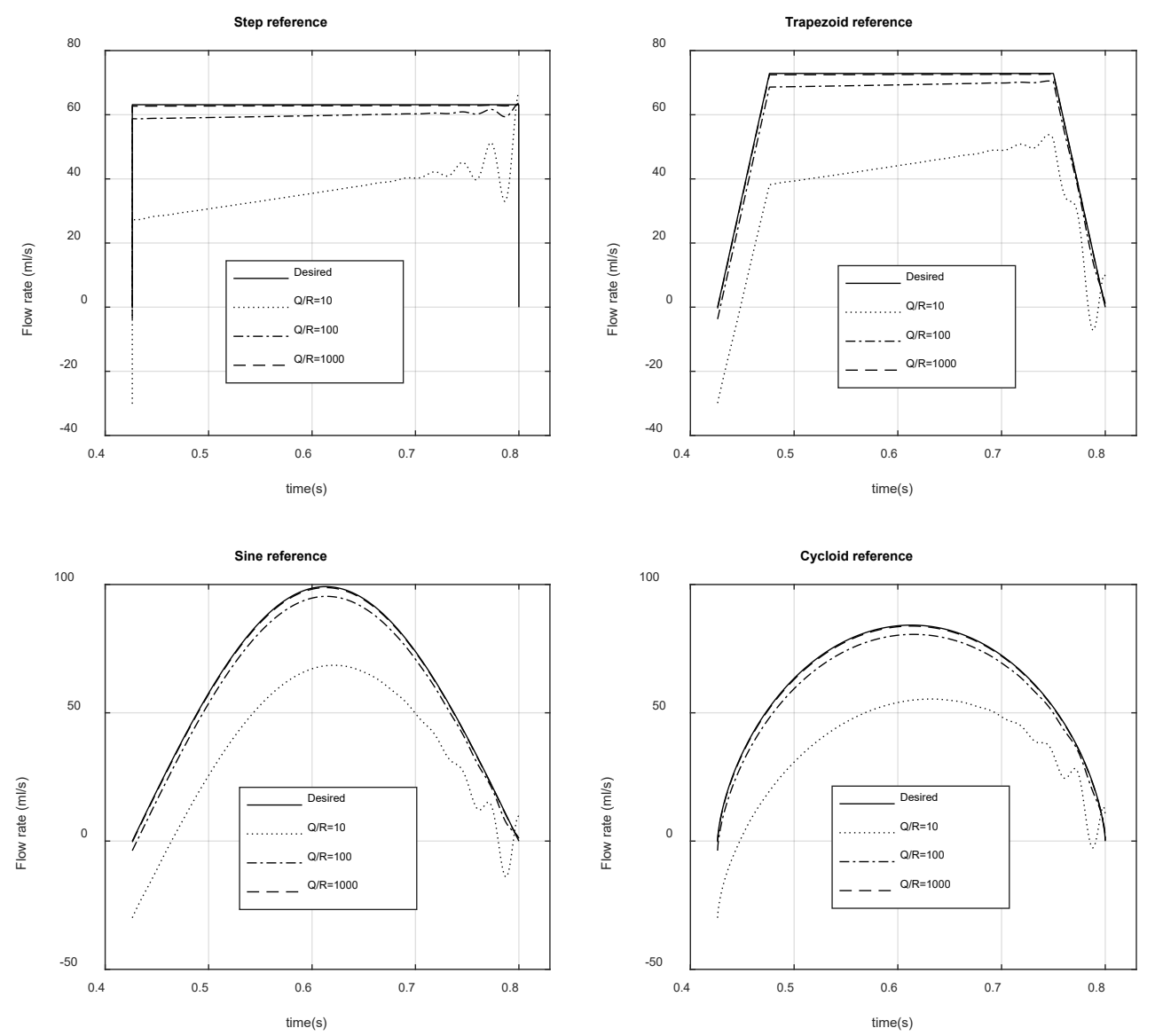

Figure 5.2. Performance of the optimal controller in tracking a desired signal. Four reference signals were tracked: step, trapezoidal, sinusoidal, and cycloidal. Solid lines represent desired signals $z(t)$, whereas dashed lines represent actual tracking signals $y(t)$ at different $Q / R$ ratios. 


\begin{tabular}{|c|c|c|c|c|c|c|c|c|}
\hline \multirow{2}{*}{$\begin{array}{c}\boldsymbol{Q} / \boldsymbol{R} \\
\text { ratio }\end{array}$} & \multicolumn{2}{|c|}{ Step } & \multicolumn{2}{|c|}{ Trapezoid } & \multicolumn{2}{c|}{ Sine } & \multicolumn{3}{c|}{ Cycloid } \\
\cline { 2 - 10 } & $\begin{array}{c}\text { Discharged } \\
\text { blood } \\
(\mathrm{ml})\end{array}$ & $\%$ & $\begin{array}{c}\text { Discharged } \\
\text { blood } \\
(\mathrm{ml})\end{array}$ & $\%$ & $\begin{array}{c}\text { Discharged } \\
\text { blood } \\
(\mathrm{ml})\end{array}$ & $\%$ & $\begin{array}{c}\text { Discharged } \\
\text { blood } \\
(\mathrm{ml})\end{array}$ & $\%$ \\
\hline $\mathbf{1}$ & -11.71 & -49.6 & -11.77 & -49.88 & -11.82 & -50.08 & -11.79 & -49.95 \\
\hline $\mathbf{1 0}$ & 13.58 & 57.54 & 13.54 & 57.36 & 13.51 & 57.26 & 13.53 & 57.32 \\
\hline $\mathbf{1 0 0}$ & 22.356 & 94.73 & 22.35 & 94.7 & 22.348 & 94.69 & 22.35 & 94.7 \\
\hline $\mathbf{1 0 0 0}$ & 23.47 & 99.46 & 23.47 & 99.46 & 23.47 & 99.46 & 23.47 & 99.46 \\
\hline $\mathbf{1 0 0 0 0}$ & 23.588 & 99.95 & 23.587 & 99.94 & 23.587 & 99.94 & 23.587 & 99.94 \\
\hline $\mathbf{1 0 0 0 0 0}$ & 23.599 & 99.99 & 23.599 & 99.99 & 23.599 & 99.99 & 23.599 & 99.99 \\
\hline $\mathbf{1 0 0 0 0 0 0}$ & 23.6 & 99.999 & 23.6 & 99.999 & 23.6 & 99.999 & 23.6 & 99.999 \\
\hline
\end{tabular}

Table 5.1. Comparison of optimal control performance in tracking desired signals using different $Q / R$ ratios.

Figure 5.3 shows the actual "optimal" control signal $U(t)$ that drives the system in order to track the desired signals when the ratio of $\mathrm{Q} / \mathrm{R}$ is 1000 (i.e. actual flow rate signal almost overlapping the desired one as shown in Figure 5.2). This control signal represents a voltage supply (if we consider Figure 4.3) or pressure source (if we consider Figure 4.1). 

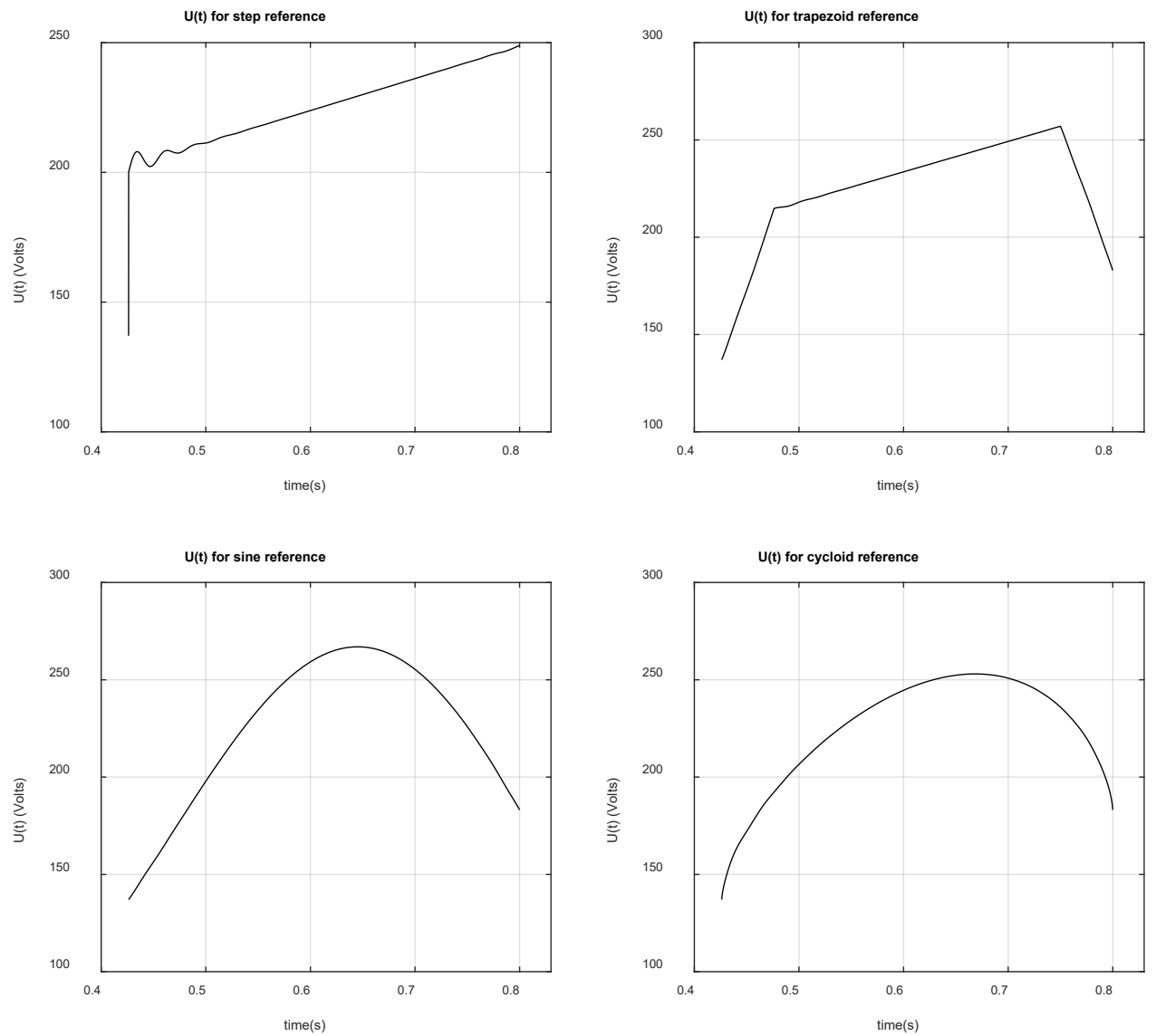

Figure 5.3. Waveform profiles for the control input $U(t)$ that drives the system to track a specific desired reference trajectory. 


\section{CHAPTER 6 Conclusions}

\subsection{Conclusions}

Hypertension contributes to cardiovascular morbidity and mortality worldwide. While many hypertensive patients respond to drug therapy, a growing number of these cases are referred to as resistant hypertension (RH), when patients cannot control their blood pressure at goal levels despite the use of multiple antihypertensive medications, including a diuretic. There are approximately 14.4 million people in America that are diagnosed with $\mathrm{RH}$, and so the research in this field may offer an opportunity to improve life for millions of RH patients.

There is no existing treatment option for $\mathrm{RH}$ that deals with altering the way in which the heart pumps blood into the aorta. The underlying hypothesis that RH may be controlled by altering the way blood is pumped into the aorta remains untested. We introduced a novel idea of implementing what we called the accumulator device that is represented by the conceptual schematic diagram in Figure 4.1. The proposed accumulator device is aimed to regulate the blood ejection from the LV into the aorta in such a way to normalize the systolic pressure below maximum levels, without reducing the cardiac output. It is our hope that this new approach proves to become a successful option for treating $\mathrm{RH}$ in the future.

The simulation work related to this novel idea revealed promising preliminary results. In one of the simulation cases (that corresponds to $R_{i}=0.5 \Omega$ ), the implementation of the accumulator device idea to treat an unhealthy condition caused a significant 
reduction of $23.7 \mathrm{mmHg}(\sim 14 \%)$ in the systolic pressure, while maintaining the $\mathrm{CO}$ almost unchanged (Table 4.3). This case corresponds to accumulating an amount of $36.1 \mathrm{ml}$ $(\sim 58 \%)$ of the $62.05 \mathrm{ml} \mathrm{SV}$, which was discharged by the accumulator back to the aorta during the filling phase, while that the remaining $(25.96 \mathrm{ml})$ was ejected naturally through the aortic valve during the ejection phase.

There are several insights that we can report based on the performed simulation work. We report that as the accumulator's inlet resistance decreases (i.e. allowing for more blood accumulation), systolic pressure decreases, and diastolic pressure increases. In other words, as blood accumulation increases (equivalent to decreasing $R_{i}$ ), the pulse pressure (difference between systolic and diastolic pressures) decreases. However, this relationship reveres when blood accumulation becomes too large, such as cases when $R_{i}=0.25$ and 0.15 $\Omega$ in Table 4.3. This is because we are pressurizing the aorta much more during diastole by delivering a large amount of accumulated blood while ignoring utilizing ejecting reasonable amounts of blood through the aortic valve during the systolic phase.

Another important insight relates to end-diastolic volume (EDV) which decreases as $R_{i}$ decreases (i.e. blood accumulation increases). We predict that this decrease in EDV creates the necessary balance to maintain the $\mathrm{CO}$ unchanged. The addition of an extra path by the accumulator for the blood leaving the LV should facilitate pumping the blood out of the LV during contraction, and this is expected to increase the CO. However, according to Frank-Starling law, as EDV decreases, stretching in the LV muscle decreases which results in lesser contraction force and consequently, a lesser amount of blood ejection. Therefore, the decrease in EDV (i.e. lesser contraction force) serves as a compensation for 
the facilitation of the blood leaving the LV due to the addition of the extra path, and consequently, $\mathrm{CO}$ is maintained almost unaltered.

A third insight relates to the influence of implementing the accumulator device idea on modulating the duration of cardiac cycle phases. We report that as $R_{i}$ decreases (i.e. allowing for more blood accumulation), the ejection phase duration decreases and the isovolumic contraction phase duration increases. The decrease in the ejection phase duration could possibly be due to creating the new path by the accumulator, which is expected to accelerate the process of pumping blood out of the LV, which consequently causes the aortic valve to close in a shorter duration of time. The increase in the isovolumic contraction phase could possibly be because the diastolic pressure increases as $R_{i}$ decreases, which means that the LV will need to wait for a longer time until the pressure inside its chamber exceeds that in the aorta (which is the diastolic pressure in normal conditions), before that the aortic valve opens.

The last insight relates to investigating the response of aortic pressure and flow due to applying different waveform shapes for the flow rate signal of the blood that is discharged by the accumulator back to the aorta during the filling phase. Among the four tested flow rate signals, the step function did not seem to be practical due to the oscillatory behavior shown in the waveform profile of the resulting aortic pressure. The trapezoidal, sinusoidal, and cycloidal-like functions of flow rate signals performed well compared to the step function flow rate signal.

Optimal control theory was then applied, using the linear quadratic tracking technique, to design a controller that can drive the control input such that the accumulator discharges blood according to a desired trajectory of flow rate. The simulation work shows 
that the designed controller performs well when the ratio of the error weighted coefficient to the control weighted coefficient $(Q / R)$ is large.

\subsection{Future Work}

Our recommendations for future research will be summarized in bullet points as follows:

- The ultimate goal of this research work is to convert the accumulator device idea into a real, physical device, and we hope that it becomes a successful treatment option for $\mathrm{RH}$ patients in the future.

- This requires continued development of this idea and through planning of designing and building a device for in-vivo experiments.

- Extending the accumulator device idea such that it adapts to changes in the heart rate.

- The use of switches in the accumulator's inlet and outlet was for demonstration purposes; however, this might not be practical, and so one should think of better alternatives.

- The accumulator represented by the schematic diagram in Figure 4.1 was of a very basic design and was just for illustration purposes; however, we should think of better and more practical accumulator designs.

- Energy dissipation by the accumulator's inlet and outlet resistances $\left(R_{i}\right.$ and $\left.R_{o}\right)$ should be investigated. 
- The current designed controller requires sensing some of the system's states such as the aortic pressure; we may want to think of other design methods, such as the Kalman filter and others that may limit the need for using sensors.

- It will be beneficial to investigate the sizing of the actual accumulator device such that it is customized based on the patient's need, in the sense that it is fabricated in the smallest size possible.

- Cardiac contractility modulation (CCM), which can modulate the contraction force of heart muscle, represents a promising modern technology for treating heart failure patients, especially those with reduced ejection fraction. Although experimental work related to $\mathrm{CCM}$ revealed that negative-amplitude signals showed to decrease the contractile force, which accordingly should decrease pressure and $\mathrm{CO}$, researchers' focus is just targeting the positive signals since they seek to enhance the contractile force to improve the ejection fraction in heart failure patients. We believe that the application of negative CCM signals is worthy to investigate if the patient's body can sacrifice lowering the $\mathrm{CO}$ for the sake of reducing blood pressure measurements. The preliminary analysis done in chapter 3 showed that the application of negative CCM signals reduced $\mathrm{CO}$ and pressure readings. Therefore, it might be worthy to consider investigating the incorporation of negative CCM signals application (which mainly works on reducing blood pressure by reducing the $\mathrm{CO}$ ) with the accumulator device idea (which mainly works on reducing more the systolic blood pressure by reducing the pulse pressure while maintain MAP) in the future work of such research direction.

- The left-ventricular assist device (LVAD) has become an increasingly prevalent therapy for long-term use (destination therapy) as an alternative to a heart transplant in 
heart failure patients. To the best of our knowledge, there is no work in the literature that utilized the use of this technology in treating resistant hypertension. We anticipate that the LVAD could serve as a possible treatment candidate for RH by designing a controlled pump that can regulate the flow of blood in such a way to achieve a desired reference signal of blood pressure while maintaining a desired $\mathrm{CO}$. Therefore, it might also be worthy to investigate this research direction. 


\title{
GLOSSARY
}

(definitions are taken from Wikipedia)

\begin{abstract}
Absolute refractory: The period immediately following the firing of a nerve fiber when it cannot be stimulated no matter how great the applied stimulus is.

Action potential: $\quad$ A brief change in voltage (membrane potential) across the cell membrane of heart cells.

Afterload: $\quad$ The stress in the wall of the left ventricle during ejection. In other word, it is the end load against which the heart contracts to eject blood.
\end{abstract}

Aorta:

The main artery in the human body, originating from the left ventricle of the heart and extending down to the abdomen, where it splits into two smaller arteries.

Aortic valve:

A valve in the human heart between the left ventricle and the aorta.

Artery:

A blood vessel that takes blood from the heart to all parts of the body.

Atrium (right and

The upper chamber in the heart that receives blood. When the left): heart muscle contracts, it pumps blood to the ventricle.

Baroreceptor reflex: One of the body's homeostatic mechanisms that helps to maintain blood pressure at nearly constant levels. It provides a rapid negative feedback loop in which an elevated blood pressure reflexively causes heart rate and blood pressure to both decrease; similarly, decreased blood pressure decreases baroreflex activation and causes heart rate to increase and to restore blood pressure levels.

Cardiac cycle: $\quad$ The sequence of mechanical and electrical events that repeats with every heartbeat.

Cardiac diastole: The part of the cardiac cycle when the heart refills with blood following systole.

Cardiac output: The volume of blood being pumped by the heart per minute.

Cardiac systole: The part of the cardiac cycle when a heart chamber contracts. 
Carotid artery:

Contractility:

Compliance:

Destination therapy:

\author{
Diastolic blood \\ pressure:
}

Ejection fraction

(EF):

Heart rate:

Hemodynamics:

Inertance:

Kirchhoff's circuit

laws:

Mitral valve:

Preload:

Pulse pressure:
An artery on each side of the head and neck supplying blood to the brain.

The innate ability of the heart muscle to contract.

The ability of a hollow organ (vessel) to distend and increase volume with increasing transmural pressure or the tendency of a hollow organ to resist recoil toward its original dimensions on application of a distending or compressing force. It is the reciprocal of "elastance".

A therapy that is final rather than being a transitional stage until another therapy.

Minimum pressure in the arteries, which occurs near the beginning of the cardiac cycle when the ventricles are filled with blood.

The fraction of blood ejected from a ventricle of the heart in each heartbeat.

The speed of the heartbeat measured by the number of contractions of the heart per minute (bpm).

The dynamics of blood flow that explain the physical laws that govern the flow of blood in the blood vessels.

A measure of the pressure difference in a fluid required to cause a unit change in the rate of change of volumetric flowrate with time.

Two equalities that deal with the current and voltage in the lumped element model of electrical circuits.

A valve that lies between the left atrium and the left ventricle.

The initial stretching of the cardiomyocytes prior to contraction. Parameters such as ventricular end diastolic volume or pressure are typical measurements for describing preload.

The difference between the systolic and diastolic pressure readings. 
Sinus (SA) node: Known as the heart's natural pacemaker. It is a group of specialized cells located in the wall of the right atrium that have the ability to spontaneously produce electrical pulses (action potentials) that travel through the heart's conduction system to activate heart contraction.

Stroke volume (SV): The volume of blood pumped from the left ventricle per beat. Systolic blood The highest blood pressure measured in a blood vessel. It pressure: occurs when the heart contracts with each heartbeat.

Vein: Blood vessels that carry blood toward the heart.

Ventricle (right and The pumping chambers of the heart.

left): 


\section{REFERENCES}

[1] S. J. Taler, S. C. Textor, and J. E. Augustine, "Resistant hypertension: comparing hemodynamic management to specialist care," Hypertension, vol. 39, no. 5, pp. 982-988, 2002.

[2] E. J. Benjamin et al., "Heart disease and stroke statistics-2017 update: a report from the American Heart Association," Circulation, vol. 135, no. 10, pp. e146e603, 2017.

[3] K. A. Illig et al., "An implantable carotid sinus stimulator for drug-resistant hypertension: surgical technique and short-term outcome from the multicenter phase II Rheos feasibility trial," Journal of vascular surgery, vol. 44, no. 6, pp. 1213-1218. e1, 2006.

[4] N. Manring, Hydraulic control systems. Wiley, 2005.

[5] N. D. Manring, Fluid power pumps and motors: analysis, design and control. McGraw Hill Professional, 2013.

[6] N. C. Tehsam, "The electrical activity of the heart and the Van Der Pol equation as its mathematical model."

[7] S. Hans and J. P. Reilly, "Resistant hypertension in 2017," Current opinion in cardiology, vol. 32, no. 4, pp. 389-396, 2017.

[8] S. D. Navaneethan, T. E. Lohmeier, and J. D. Bisognano, "Baroreflex stimulation: A novel treatment option for resistant hypertension," Journal of the American Society of Hypertension, vol. 3, no. 1, pp. 69-74, 2009.

[9] A. Carlsten, B. FOLKOW, G. Grimby, and C. A. HAMBERGER, "Cardiovascular effects of direct stimulation of the carotid sinus nerve in man," Acta Physiologica, vol. 44, no. 2, pp. 138-145, 1958.

[10] J. P. Reilly and C. J. White, "Renal Denervation for Resistant Hypertension," Progress in Cardiovascular Diseases, vol. 59, no. 3, pp. 295-302, 2016.

[11] A. E. Burchell, M. D. Lobo, N. Sulke, P. A. Sobotka, and J. F. R. Paton, "Arteriovenous anastomosis: Is this the way to control hypertension?," Hypertension, vol. 64, no. 1, pp. 6-12, 2014.

[12] M. D. Lobo et al., "Central arteriovenous anastomosis for the treatment of patients with uncontrolled hypertension (the ROX CONTROL HTN study): a randomised controlled trial," The Lancet, vol. 385, no. 9978, pp. 1634-1641, 2015. 
[13] Y.-C. Yu, "Mathematical modeling of the cardiovascular system and its control mechanisms," ed: Oxford, UK: Eolss Publishers, 2011.

[14] S. A. Haddad and W. A. Serdijn, "The evolution of pacemakers: An electronics perspective," Ultra low-power biomedical signal processing, pp. 13-31, 2009.

[15] D. J. Goldstein, M. C. Oz, and E. A. Rose, "Implantable Left Ventricular Assist Devices," New England Journal of Medicine, vol. 339, no. 21, pp. 1522-1533, 1998/11/19 1998 .

[16] D. Mancini and P. C. Colombo, "Left ventricular assist devices: a rapidly evolving alternative to transplant," Journal of the American College of Cardiology, vol. 65, no. 23, pp. 2542-2555, 2015.

[17] E. A. Rose et al., "Long-term use of a left ventricular assist device for end-stage heart failure," New England Journal of Medicine, vol. 345, no. 20, pp. 1435-1443, 2001.

[18] M. VanAntwerp and J. R. Hogness, The artificial heart: prototypes, policies, and patients. National Academies Press, 1991.

[19] T. G. Mesana, "Rotary blood pumps for cardiac assistance: a "must"?," (in eng), Artif Organs, vol. 28, no. 2, pp. 218-25, Feb 2004.

[20] J. A. Larose, D. Tamez, M. Ashenuga, and C. Reyes, "Design concepts and principle of operation of the HeartWare ventricular assist system," (in eng), Asaio j, vol. 56, no. 4, pp. 285-9, Jul-Aug 2010.

[21] A. Cheng, C. A. Williamitis, and M. S. Slaughter, "Comparison of continuousflow and pulsatile-flow left ventricular assist devices: is there an advantage to pulsatility?," Annals of cardiothoracic surgery, vol. 3, no. 6, pp. 573-581, 2014.

[22] G. B. Bearnson, D. B. Olsen, P. S. Khanwilkar, J. W. Long, P. E. Allaire, and E. H. Maslen, "Pulsatile operation of a centrifugal ventricular assist device with magnetic bearings," Asaio Journal, vol. 42, no. 5, pp. M620-623, 1996.

[23] F. Abi-Samra and D. Gutterman, "Cardiac contractility modulation: a novel approach for the treatment of heart failure," Heart failure reviews, vol. 21, no. 6, pp. 645-660, 2016.

[24] A. R. Lyon, M. A. Samara, and D. S. Feldman, "Cardiac contractility modulation therapy in advanced systolic heart failure," Nature Reviews Cardiology, vol. 10, no. 10 , pp. 584-598, 2013.

[25] H. Burri and P. Bordachar, "Cardiac contractility modulation for treatment of heart failure," Cardiovascular Medicine, vol. 16, no. 10, pp. 259-262, 2013. 
[26] D. M. Bers, "Cardiac excitation-contraction coupling," Nature, vol. 415, no. 6868, pp. 198-205, 2002.

[27] A. M. Katz and B. H. Lorell, "Regulation of cardiac contraction and relaxation," Circulation, vol. 102, no. suppl 4, pp. Iv-69-Iv-74, 2000.

[28] E. Woods, R. Heppner, and S. Weidmann, "Inotropic effects of electric currents. 1. Positive and negative effects of constant electrical currents or current pulses applied during cardiac action potentials. II. Hypothesis: Calcium movements, excitation-contraction coupling in inotropic effects," Circulation Research, vol. 24, pp. 409-445, 1969.

[29] D. Burkhoff et al., "Electric currents applied during the refractory period can modulate cardiac contractility in vitro and in vivo," Heart failure reviews, vol. 6, no. 1, pp. 27-34, 2001.

[30] C. Pappone et al., "Cardiac contractility modulation by electric currents applied during the refractory period in patients with heart failure secondary to ischemic or idiopathic dilated cardiomyopathy," The American journal of cardiology, vol. 90, no. 12, pp. 1307-1313, 2002.

[31] S. Mohri et al., "Cardiac contractility modulation by electric currents applied during the refractory period," American Journal of Physiology-Heart and Circulatory Physiology, vol. 282, no. 5, pp. H1642-H1647, 2002.

[32] D. Burkhoff and S. A. Ben-Haim, "Nonexcitatory electrical signals for enhancing ventricular contractility: rationale and initial investigations of an experimental treatment for heart failure," American Journal of Physiology - Heart and Circulatory Physiology, 10.1152/ajpheart.01311.2004 vol. 288, no. 6, p. H2550, 2005.

[33] C. B. Brunckhorst, I. Shemer, Y. Mika, S. A. Ben-Haim, and D. Burkhoff, "Cardiac contractility modulation by non-excitatory currents: studies in isolated cardiac muscle," European journal of heart failure, vol. 8, no. 1, pp. 7-15, 2006.

[34] B. Van Der Pol and J. Van Der Mark, "The heartbeat considered as a relaxation oscillation, and an electrical model of the heart," The London, Edinburgh, and Dublin Philosophical Magazine and Journal of Science, vol. 6, no. 38, pp. 763775, 1928.

[35] A. L. Hodgkin and A. F. Huxley, "A quantitative description of membrane current and its application to conduction and excitation in nerve," The Journal of physiology, vol. 117, no. 4, pp. 500-544, 1952.

[36] R. FitzHugh, "Impulses and physiological states in theoretical models of nerve membrane," Biophysical journal, vol. 1, no. 6, pp. 445-466, 1961. 
[37] J. Abrams, E. Irwin, C. Walvatne, L. Segar, J. Foker, and F. Cerra, "Use of a modified van den Pol's oscillator to construct ventricular pressure-volume relations," Journal of cardiothoracic and vascular anesthesia, vol. 7, no. 2, pp. 195-199, 1993.

[38] K. Grudziński and J. J. Żebrowski, "Modeling cardiac pacemakers with relaxation oscillators," Physica A: Statistical Mechanics and its Applications, vol. 336, no. 1, pp. 153-162, 2004.

[39] Y. Shi, P. Lawford, and R. Hose, "Review of zero-D and 1-D models of blood flow in the cardiovascular system," Biomedical engineering online, vol. 10:33, 2011.

[40] N. Westerhof, J.-W. Lankhaar, and B. E. Westerhof, "The arterial windkessel," Medical \& biological engineering \& computing, vol. 47, no. 2, pp. 131-141, 2009.

[41] M. Capoccia, "Development and characterization of the arterial windkessel and its role during left ventricular assist device assistance," Artificial organs, vol. 39, no. $8,2015$.

[42] O. Frank, "Die Grundform des arteriellen Pulses," Z. Biol-Munich, vol. 37, pp. 483-526, 1899.

[43] N. Westerhof, F. Bosman, C. J. De Vries, and A. Noordergraaf, "Analog studies of the human systemic arterial tree," Journal of Biomechanics, vol. 2, no. 2, pp. 121-143, 1969.

[44] D. Burkhoff, J. Alexander, and J. Schipke, "Assessment of Windkessel as a model of aortic input impedance," American Journal of Physiology-Heart and Circulatory Physiology, vol. 255, no. 4, pp. H742-H753, 1988.

[45] N. Westerhof, G. Elzinga, and P. Sipkema, "An artificial arterial system for pumping hearts," Journal of applied physiology, vol. 31, no. 5, pp. 776-781, 1971.

[46] N. Stergiopulos, B. E. Westerhof, and N. Westerhof, "Total arterial inertance as the fourth element of the windkessel model," American Journal of PhysiologyHeart and Circulatory Physiology, vol. 276, no. 1, pp. H81-H88, 1999.

[47] G. Ortiz-León, M. Vílchez-Monge, and J. J. Montero-Rodríguez, "Simulations of the Cardiovascular System Using the Cardiovascular Simulation Toolbox," in OASIcs-OpenAccess Series in Informatics, 2014, vol. 36: Schloss DagstuhlLeibniz-Zentrum fuer Informatik.

[48] M. A. Simaan, G. Faragallah, Y. Wang, and E. Divo, "Left ventricular assist devices: Engineering design considerations," in New Aspects of Ventricular Assist Devices: InTech, 2011. 
[49] K. Ohuchi et al., "Control strategy for rotary blood pumps," Artificial organs, vol. 25, no. 5, pp. 366-370, 2001.

[50] A. Ferreira, S. Chen, D. G. Galati, M. A. Simaan, and J. F. Antaki, "A dynamical state space representation and performance analysis of a feedback controlled rotary left ventricular assist device," American Society of Mechanical Engineers, Dynamic Systems and Control Division (Publication) DSC, vol. 74 DSC, 2005.

[51] M. A. Simaan, A. Ferreira, S. Chen, J. F. Antaki, and D. G. Galati, "A dynamical state space representation and performance analysis of a feedback-controlled rotary left ventricular assist device," IEEE Transactions on Control Systems Technology, vol. 17, no. 1, pp. 15-28, 2009.

[52] H. U. Fuchs, The dynamics of heat: a unified approach to thermodynamics and heat transfer. Springer Science \& Business Media, 2010.

[53] J. R. Levick, An introduction to cardiovascular physiology. ButterworthHeinemann, 2013.

[54] V. Creigen et al., "Modeling a heart pump," European Study Group Mathematics with Industry, p. 7, 2007.

[55] D. Burkoff, "Mechanical properties of the heart and its interaction with the vascular system," Cardiac Physiology, pp. 1-23, 2002.

[56] A. Ferreira, S. Chen, M. A. Simaan, J. R. Boston, and J. F. Antaki, "A nonlinear state-space model of a combined cardiovascular system and a rotary pump," in Decision and Control, 2005 and 2005 European Control Conference. CDCECC'05. 44th IEEE Conference on, 2005, pp. 897-902: IEEE.

[57] E. B. Shim, J. Y. Sah, and C. H. Youn, "Mathematical modeling of cardiovascular system dynamics using a lumped parameter method," The Japanese journal of physiology, vol. 54, no. 6, pp. 545-553, 2004.

[58] A. M. Katz, Physiology of the Heart. Lippincott Williams \& Wilkins, 2010.

[59] H. Suga and K. Sagawa, "Instantaneous pressure-volume relationships and their ratio in the excised, supported canine left ventricle," Circulation research, vol. 35 , no. 1, pp. 117-126, 1974.

[60] N. Stergiopulos, J. J. Meister, and N. Westerhof, "Determinants of stroke volume and systolic and diastolic aortic pressure," The American journal of physiology, vol. 270, pp. H2050-H2059, 1996.

[61] M. A. Simaan, "Rotary heart assist devices," in Springer Handbook of Automation: Springer, 2009, pp. 1409-1422. 
[62] A. Guyton and J. Hall, "Textbook of medical physiology, 11th," ed: Elsevier Inc., 2006.

[63] K. R. Walley, "Left ventricular function: time-varying elastance and left ventricular aortic coupling," Critical Care, vol. 20, no. 1, p. 270, 2016.

[64] E. Kung et al., "A simulation protocol for exercise physiology in Fontan patients using a closed loop lumped-parameter model," Journal of biomechanical engineering, vol. 136, no. 8, p. 081007, 2014.

[65] H. J. Sussmann and J. C. Willems, "300 years of optimal control: from the brachystochrone to the maximum principle," IEEE Control Systems, vol. 17, no. 3, pp. 32-44, 1997.

[66] D. S. Naidu, Optimal control systems. CRC press, 2002.

[67] V. Boltyanskii, R. Gamkrelidze, and L. Pontryagin, "Towards a theory of optimal processes, Reports Acad," Sci. USSR, vol. 110, no. 1, p. 710, 1956.

[68] A. Alturki, F. Feng, A. Nair, V. Guntu, and S. S. Nair, "Distinct current modules shape cellular dynamics in model neurons," Neuroscience, vol. 334, pp. 309-331, 2016.

[69] A. Alturki, A. Nair, V. Guntu, and S. S. Nair, "Single neuron models for network simulations," presented at the Indian control conference, Chennai, India, 2015.

[70] V. Guntu, F. Feng, A. Alturki, A. Nair, P. Samarth, and S. S. Nair, "Amygdala Models," in Computational Models of Brain and Behavior, 2017, pp. 285-301. 


\section{APPENDIX}

In this section, we show the derivation work of the equations used in chapter 5 to solve for the linear quadratic tracking problem. Optimal control textbooks present the necessary equations needed to solve the LQT problem by applying the Pontryagin Minimum Principle based on the following linear, time-varying system:

$$
\begin{aligned}
& \dot{x}(t)=A(t) x(t)+B(t) u(t) \\
& y(t)=C(t) x(t)
\end{aligned}
$$

However, our system in equation (4.22) is in the form:

$$
\begin{aligned}
& \dot{x}(t)=A(t) x(t)+B(t) u(t) \\
& y(t)=C(t) x(t)+D(t) u(t)
\end{aligned}
$$

with the additional term $D(t)$, which makes significant changes in the derived equations. Below, we will apply the Pontryagin Minimum Principle based on the system that has the feedforward term $D(t)$. Since our system in (4.22) is a single-input single-output, which means that $D(t)$ is scalar, with the input vector $B(t)$, output vector $C(t)$, and feedforward term $D(t)$ being time invariant, the system in (4.22) can be re-written, for the sake of simplicity, according to the following:

$$
\begin{aligned}
& \dot{x}(t)=A(t) x(t)+B u(t) \\
& y(t)=C x(t)+d u(t)
\end{aligned}
$$

We first start by defining the error $e(t)$, which is a scalar function, according to the following:

$$
e(t)=z(t)-y(t)
$$


Which can be re-written as:

$$
e(t)=z(t)-C x(t)-d u(t)
$$

We then choose our performance index according to the following:

$$
J=\frac{1}{2} e^{T}\left(t_{f}\right) F\left(t_{f}\right) e\left(t_{f}\right)+\frac{1}{2} \int_{t_{0}}^{t_{f}}\left[e^{T}(t) Q(t) e^{T}(t)+u^{T}(t) R(t) u(t)\right] d t
$$

Since $Q(t)$ and $R(t)$ are scalar functions that will be chosen to be constant values, then $Q(t), R(t)$, and $F\left(t_{f}\right)$ are represented by the scalars $q, r$, and $f$, respectively. Thus, the performance index can be written as:

$$
J=\frac{1}{2} f\left[e\left(t_{f}\right)\right]^{2}+\frac{1}{2} \int_{t_{0}}^{t_{f}}\left(q[e(t)]^{2}+r[u(t)]^{2}\right) d t
$$

Step 1: Formulating the Hamiltonian function:

$$
\begin{aligned}
H(x(t), u(t), \lambda(t)) & \\
& =\frac{1}{2} q[z(t)-C x(t)-d u(t)]^{2}+\frac{1}{2} r[u(t)]^{2} \\
& +\lambda^{T}(t)[A(t) x(t)+B u(t)]
\end{aligned}
$$

Step 2: Finding the open-loop optimal control from the Hamiltonian:

$$
\frac{d H}{d u}=0 \longrightarrow q *(-d) *[z(t)-C x(t)-d u(t)]+r u(t)+B^{T} \lambda(t)=0
$$


We can now write the equation for the optimal control:

$$
u^{*}(t)=\frac{1}{r+q d^{2}}\left[-q d C x^{*}(t)-B^{T} \lambda^{*}(t)+q d z(t)\right]
$$

Step 3: Finding the state and co-state systems from the Hamiltonian:

$$
\dot{x}(t)=\frac{d H}{d \lambda}=A(t) x(t)+B u(t)
$$

Plugging in the expression for $u^{*}(t)$ from equation (A4) into the above equation to find the optimal state equation, we obtain:

$$
\dot{x}^{*}(t)=\left[A(t)-\frac{q d}{r+q d^{2}} B C\right] x^{*}(t)-\frac{B B^{T}}{r+q d^{2}} \lambda^{*}(t)+\frac{d q}{r+q d^{2}} B z(t)
$$

The optimal co-state equation can be derived as follows:

$$
\dot{\lambda}^{*}(t)=-\frac{d H}{d x}=-\left[-q C^{T}\left[z(t)-C x^{*}(t)-d u^{*}(t)\right]+A^{T}(t) \lambda^{*}(t)\right]
$$

Plugging in the expression for $u^{*}(t)$ from equation (A4) into the above equation to find the optimal co-state equation, we obtain:

$$
\begin{gathered}
\dot{\lambda}^{*}(t)=\left(\frac{q^{2} d^{2}}{r+q d^{2}} C^{T} C-q C^{T} C\right) x^{*}(t)+\left(\frac{q d}{r+q d^{2}} C^{T} B^{T}-A^{T}(t)\right) \lambda^{*}(t) \\
+\left(q C^{T}-\frac{q^{2} d^{2}}{r+q d^{2}} C^{T}\right) z(t)
\end{gathered}
$$


For the sake of simplicity, let us define:

$$
\begin{aligned}
& \alpha=\frac{q d}{r+q d^{2}} \\
& \beta=\frac{q^{2} d^{2}}{r+q d^{2}} \\
& V=\frac{1}{r+q d^{2}} B^{T}
\end{aligned}
$$

where $\alpha$ and $\beta$ are scalars and $V$ is a row vector.

Equations (A4), (A5), and (A6) can now be re-written as follows:

$$
\begin{gathered}
u^{*}(t)=-\alpha C x^{*}(t)-V \lambda^{*}(t)+\alpha z(t) \\
\dot{x}^{*}(t)=(A(t)-\alpha B C) x^{*}(t)-B V \lambda^{*}(t)+\alpha B z(t) \\
\dot{\lambda}^{*}(t)=(\beta-q) C^{T} C x^{*}(t)+\left(\alpha C^{T} B^{T}-A^{T}(t)\right) \lambda^{*}(t)+(q-\beta) C^{T} z(t)
\end{gathered}
$$

Combining the optimal state equation (A9) and optimal co-state equation (A10), we can now obtain the Hamiltonian canonical system:

$$
\left[\begin{array}{c}
\dot{x}^{*}(t) \\
\dot{\lambda}^{*}(t)
\end{array}\right]=\left[\begin{array}{cc}
A(t)-\alpha B C & -B V \\
(\beta-q) C^{T} C & \alpha C^{T} B^{T}-A^{T}(t)
\end{array}\right]\left[\begin{array}{l}
x^{*}(t) \\
\lambda^{*}(t)
\end{array}\right]+\left[\begin{array}{c}
\alpha B \\
(q-\beta) C^{T}
\end{array}\right] z(t)
$$

The above system includes $2 n$ differential equations (12 differential equations in our case since our cardiovascular system has 6 state variables).

The boundary conditions of the Hamiltonian canonical system in (A11) are given by the initial condition of the states:

$$
x\left(t=t_{0}\right)=x\left(t_{0}\right)
$$


The final condition comes from the co-states, where the final time $t_{f}$ being specified, according to the following:

$$
\begin{aligned}
\lambda\left(t_{f}\right)=\frac{\partial}{\partial x\left(t_{f}\right)} & {\left[\frac{1}{2} f\left(t_{f}\right)\left[e\left(t_{f}\right)\right]^{2}\right] } \\
& =\frac{\partial}{\partial x\left(t_{f}\right)}\left[\frac{1}{2} f\left[z\left(t_{f}\right)-C x\left(t_{f}\right)-d u\left(t_{f}\right)\right]^{2}\right] \\
& =-f C^{T}\left[z\left(t_{f}\right)-C x\left(t_{f}\right)-d u\left(t_{f}\right)\right] \\
& =f C^{T} C x\left(t_{f}\right)+f C^{T} d u\left(t_{f}\right)-f C^{T} z\left(t_{f}\right)
\end{aligned}
$$

Plugging in the expression for $u^{*}(t)$ from equation (A8) into the above equation, we get:

$$
\begin{array}{r}
\lambda^{*}\left(t_{f}\right)=f C^{T} C x^{*}\left(t_{f}\right)+f C^{T} d\left[-\alpha C x^{*}\left(t_{f}\right)-V \lambda^{*}\left(t_{f}\right)+\alpha z\left(t_{f}\right)\right]-f C^{T} z\left(t_{f}\right) \\
=f(1-\alpha d) C^{T} C x^{*}\left(t_{f}\right)-f d C^{T} V \lambda^{*}\left(t_{f}\right)-f(1-\alpha d) C^{T} z\left(t_{f}\right)
\end{array}
$$

Which can be written as:

$$
\begin{aligned}
\lambda^{*}\left(t_{f}\right)=(I+ & \left.f d C^{T} V\right)^{-1} f(1-\alpha d) C^{T} C x^{*}\left(t_{f}\right) \\
& -\left(I+f d C^{T} V\right)^{-1} f(1-\alpha d) C^{T} z\left(t_{f}\right)
\end{aligned}
$$

where $I$ is the identity matrix of size $6 \times 6$.

Step 4: Finding Riccati and Vector equations: the co-state boundary condition in (A12) and the solution of the Hamiltonian canonical system in (A11) indicate that there is a relationship between the state and co-state according to the following:

$$
\lambda^{*}(t)=P(t) x^{*}(t)-g(t)
$$


where $P(t)$ is an $n \times n$ symmetric matrix (6x6 in our cardiovascular model), and $g(t)$ is an $n \mathrm{x} 1$ vector ( $6 \mathrm{x} 1$ in our case), both need to be determined so as to satisfy the system in (A11). This can be accomplished by deriving two key equations called differential Riccati equation (DRE) and vector differential equation, which will be shown next.

Starting with differentiating (A13) with respect to time, we obtain:

$$
\dot{\lambda}^{*}(t)=\dot{P}(t) x^{*}(t)+P(t) \dot{x}^{*}(t)-\dot{g}(t)
$$

Substituting for $\dot{x}^{*}(t), \dot{\lambda}^{*}(t)$, and $\lambda^{*}(t)$ from (A9), (A10), and (A13); respectively, into (A14) yields the following:

$$
\begin{aligned}
(\beta-q) C^{T} C x^{*} & (t)+\left(\alpha C^{T} B^{T}-A^{T}(t)\right)\left[P(t) x^{*}(t)-g(t)\right]+(q-\beta) C^{T} z(t) \\
& =\dot{P}(t) x^{*}(t) \\
& +P(t)\left[(A(t)-\alpha B C) x^{*}(t)-B V\left(P(t) x^{*}(t)-g(t)\right)+\alpha B z(t)\right]-\dot{g}(t)
\end{aligned}
$$

Re-arranging the above equation we obtain:

$$
\begin{aligned}
& {\left[\dot{P}(t)+P(t)(A(t)-\alpha B C-B V P(t))+(q-\beta) C^{T} C+\left(A^{T}(t)-\right.\right.} \\
& \left.\left.\alpha C^{T} B^{T}\right) P(t)\right] x^{*}(\boldsymbol{t})-\left[\dot{g}(t)-\left(P(t) B V+\alpha C^{T} B^{T}-A^{T}(t)\right) g(t)-\right. \\
& \left.\left(\alpha P(t) B-q C^{T}+\beta C^{T}\right) z(t)\right]=0
\end{aligned}
$$

The relationship in equation (A15) must satisfy for all $x^{*}(t), z(t)$, and $t$. This leads us to the $n \times n P(t)$ to satisfy the matrix differential Riccati equation (DRE):

$$
\begin{gathered}
\dot{P}(t)=P(t)(\alpha B C-A(t)+B V P(t))+(\beta-q) C^{T} C \\
+\left(\alpha C^{T} B^{T}-A^{T}(t)\right) P(t)
\end{gathered}
$$


And the $n \times 1 g(t)$ vector to satisfy the vector differential equation:

$$
\dot{g}(t)=\left(P(t) B V+\alpha C^{T} B^{T}-A^{T}(t)\right) g(t)+\left(\alpha P(t) B-q C^{T}+\beta C^{T}\right) z(t)
$$

Since $P(t)$ is symmetric, equations (A16) and (A17) result in a set of $n(n+1) / 2+n$ firstorder differential equations with boundary conditions that can be inferred from equations (A12) and (A13), according to the following:

$$
\begin{gathered}
P\left(t_{f}\right)=\left(I+f d C^{T} V\right)^{-1} f(1-\alpha d) C^{T} C \\
g\left(t_{f}\right)=\left(I+f d C^{T} V\right)^{-1} f(1-\alpha d) C^{T} z\left(t_{f}\right)
\end{gathered}
$$

Therefore, the $n(n+1) / 2+n$ first-order differential equations (21 differential equations in our case) resulting from the matrix differential Riccati equation (A16) and the vector differential equation (A17) can be solved backward using the boundary conditions in (A18) and (A19).

Step 5: Finding the closed-loop optimal control by plugging in the optimal co-state (A13) into (A8) according to the following:

$$
u^{*}(t)=-\alpha C x^{*}(t)-V\left[P(t) x^{*}(t)-g(t)\right]+\alpha z(t)
$$

which can also be written as:

$$
u^{*}(t)=[-\alpha C-V P(t)] x^{*}(t)+V g(t)+\alpha z(t)
$$


Step 6: Finding the optimal state by plugging in the optimal control $u^{*}(t)$ from (A20) into the state equation in step 3, we obtain:

$$
\dot{x}(t)=A(t) x(t)+B\left([-\alpha C-V P(t)] x^{*}(t)+V g(t)+\alpha z(t)\right)
$$

Which can now be written as:

$$
\dot{x}^{*}(t)=(A(t)-\alpha B C-B V P(t)) x^{*}(t)+B V g(t)+\alpha B z(t)
$$

Recalling the definitions of $\alpha$ and $V$ in (A7) and plugging their values in (A20) and (A21), the optimal control $u^{*}(t)$ and optimal state $x^{*}(t)$ become:

$$
\begin{gathered}
u^{*}(t)=-\frac{1}{r+q d^{2}}\left[q d C x^{*}(t)+B^{T}\left(P(t) x^{*}(t)-g(t)\right)-q d z(t)\right] \\
\dot{x}^{*}(t)=\left(A(t)-\frac{q d}{r+q d^{2}} B C-\frac{1}{r+q d^{2}} B B^{T} P(t)\right) x^{*}(t) \\
+\frac{1}{r+q d^{2}} B B^{T} g(t)+\frac{q d}{r+q d^{2}} B z(t)
\end{gathered}
$$




\section{VITA}

Adel Alturki was born in Jeddah, Saudi Arabia, in 1978. He completed his Bachelor of Science degree in Electrical Engineering at King Fahd University of Petroleum and Minerals, Dhahran, Saudi Arabia in May 2000. Following his graduation, Adel was hired as a project engineer in the field of electrical power transmission for five years, before he joins another professional engineering position as an instrumentation and control maintenance engineer at a steel making plant for two years. Adel then joined a faculty position in Electrical and Electronics Engineering Department at Yanbu Industrial College in Saudi Arabia, where he was granted a scholarship to pursue his graduate studies in the United States. He attended Western Michigan University, Kalamazoo Michigan, in September 2008 where he obtained dual Master degrees, one in Electrical Engineering and another one in Applied and Computational Mathematics, both in April 2011. In August 2012, Adel attended the University of Missouri at Columbia where he joined the Computational Neurobiology Center and published a journal paper [68], a conference paper [69], co-authored a book chapter [70], and presented three conference posters. While at the University of Missouri, Adel worked as a teaching assistant for different undergraduate courses in Electrical Engineering and was honored for his dedication and exceeding expectations in teaching by receiving the TA Choice Award in May 2016. After working in the Computational Neurobiology Center, Adel switched to another lab in summer 2016 where he started the work under the supervision of Dr. Noah Manring. Adel Alturki successfully defended his dissertation and received his doctoral degree in Electrical Engineering in May 2018. 